Florida International University FIU Digital Commons

FIU Electronic Theses and Dissertations

University Graduate School

3-7-2016

\title{
Fully Decentralized Multi-Agent System for Optimal Microgrid Control
}

Ricardo de Azevedo

Florida International University, ricardo.de.azevedo@gmail.com

DOI: $10.25148 /$ etd.FIDC000263

Follow this and additional works at: https://digitalcommons.fiu.edu/etd

Part of the Controls and Control Theory Commons, Power and Energy Commons, and the Systems and Communications Commons

\section{Recommended Citation}

de Azevedo, Ricardo, "Fully Decentralized Multi-Agent System for Optimal Microgrid Control" (2016). FIU Electronic Theses and Dissertations. 2461.

https://digitalcommons.fiu.edu/etd/2461

This work is brought to you for free and open access by the University Graduate School at FIU Digital Commons. It has been accepted for inclusion in FIU Electronic Theses and Dissertations by an authorized administrator of FIU Digital Commons. For more information, please contact dcc@fiu.edu. 


\section{FLORIDA INTERNATIONAL UNIVERSITY}

Miami, Florida

FULLY DECENTRALIZED MULTI-AGENT SYSTEM FOR OPTIMAL MICROGRID CONTROL

A thesis submitted in partial fulfillment of the

requirements for the degree of

MASTER OF SCIENCE

in

ELECTRICAL ENGINEERING

by

Ricardo de Azevedo

2016 
To: $\quad$ Interim Dean Ranu Jung

College of Engineering and Computing

This thesis, written by Ricardo de Azevedo, and entitled Fully Decentralized Multi-Agent System for Optimal Microgrid Control, having been approved in respect to style and intellectual content, is referred to you for judgment.

We have read this thesis and recommend that it be approved.

Arif Selcuk Uluagac

Kemal Akkaya

Osama Mohammed, Major Professor

Date of Defense: March 7, 2016

The thesis of Ricardo de Azevedo is approved.

Interim Dean Ranu Jung College of Engineering and Computing

Andrés G. Gil

Vice President for Research and Economic Development and Dean of the University Graduate School

Florida International University, 2016 
(C) Copyright 2016 by Ricardo de Azevedo

All rights reserved. 


\section{DEDICATION}

This thesis is dedicated to my parents, who have given me endless support and the

opportunity to pursue my dreams. I am truly privileged to have such great examples to follow and my gratitude cannot be expressed in words. 


\section{ACKNOWLEDGMENTS}

I want to thank Professor Osama Mohammed who gave me the opportunity to be a researcher in his world class research group at the Energy Systems Research Laboratory. Professor Mohammed shared his ideas and wisdom and challenged me to acquire new skills and knowledge in Electric Energy Systems. Professor Mohammed guided me in learning most everything I know of Power Systems.

I also owe a special thanks to Professor Mohammed’s doctoral candidate, Mr. Mehmet Hazar Cintuglu who provided needed help in forming and experimenting with the ideas in this thesis as well as supporting me in how to operate the Smart Grid Testbed.

I would like to also thank my committee members for their insightful comments and constructive suggestions in the review of my thesis.

I also want to acknowledge all my colleagues in the research group at the Energy Systems Research Laboratory and in the department who somehow or another shared part of this journey with me. 


\section{ABSTRACT OF THE THESIS \\ FULLY DECENTRALIZED MULTI-AGENT SYSTEM FOR \\ OPTIMAL MICROGRID CONTROL}

by

Ricardo de Azevedo

Florida International University, 2016

Miami, Florida

\section{Professor Osama Mohammed, Major Professor}

In preparation for the influx of renewable energy sources that will be added to the electrical system, flexible and adaptable control schemes are necessary to accommodate the changing infrastructure. Microgrids have been gaining much attention as the main solution to the challenges of distributed and intermittent generation, but due to their low inertia, they need fast-acting control systems in order to maintain stability. Multi-Agent Systems have been proposed as dynamic control and communication frameworks. Decentralized arrangements of agents can provide resiliency and the much-desired "plug and play” behavior. This thesis describes a control system that implements droop control and the diffusion communication scheme without the need of a centralized controller to coordinate the Microgrid agents to maintain the frequency and stable operating conditions of the system. Moreover, the inter-agent communication is unaffected by changing network configurations and can achieve optimal economic dispatch through distributed optimization. 


\section{TABLE OF CONTENTS}

CHAPTER

PAGE

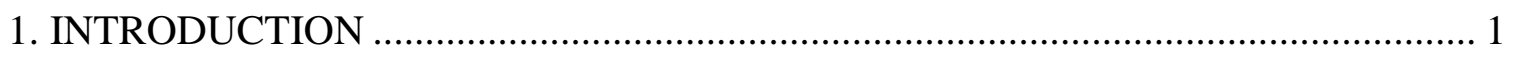

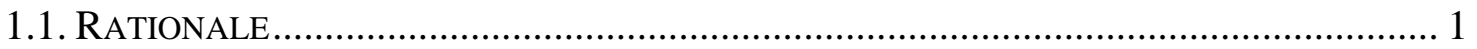

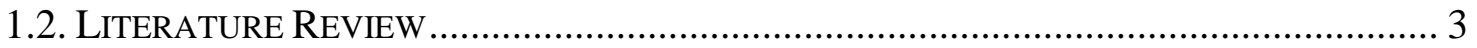

1.3. ORIGINAL CONTRIBUTIONS............................................................................ 5

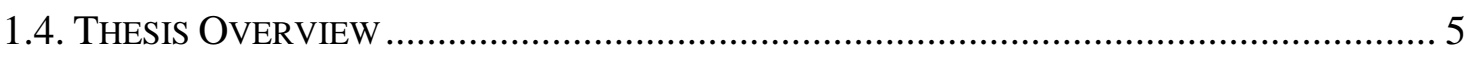

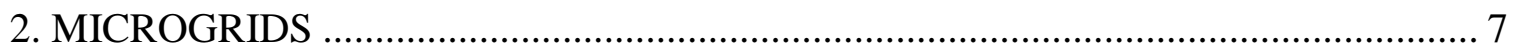

2.1. THE FORMATION OF THE ELECTRIC GRID …………................................................ 7

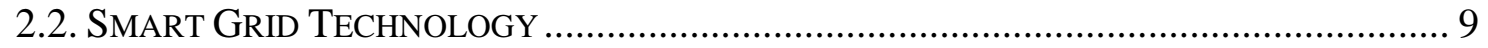

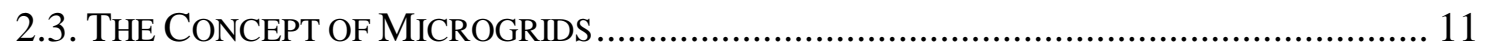

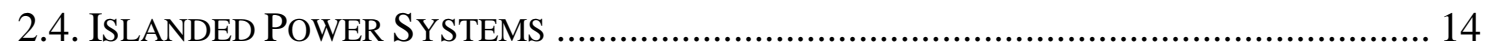

3. POWER SYSTEM OPTIMIZATION AND DROOP CONTROL ……....................... 16

3.1. INTRODUCTION TO ECONOMIC DISPATCH ……………........................................ 16

3.2. INTRODUCTION TO GENERATOR CONTROL SYSTEMS .............................................. 19

3.3. DROOP CONTROL BASICS .................................................................................. 22

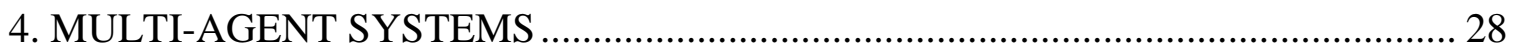

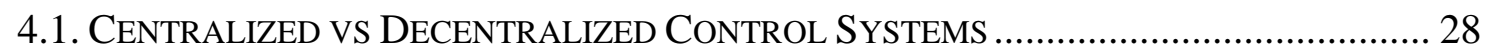

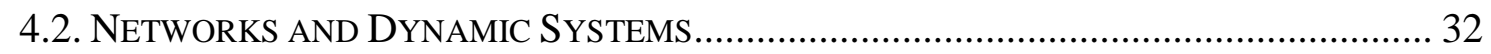

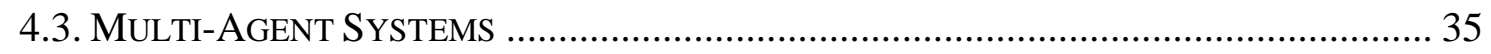

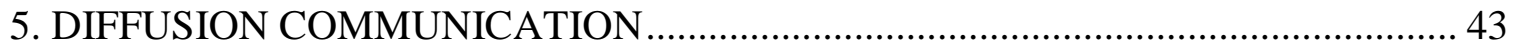

5.1. INFORMATION SHARING IN NETWORKS............................................................... 43

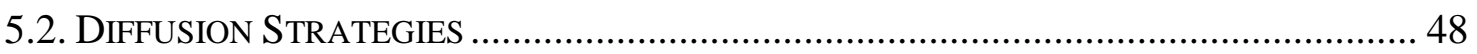

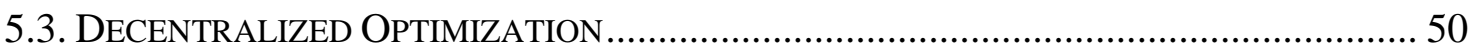

5.4. DISTRIBUTED MICROGRID OPTIMIZATION ……………....................................... 52

6. FULLY DECENTRALIZED MULTI-AGENT SYSTEM FOR OPTIMAL

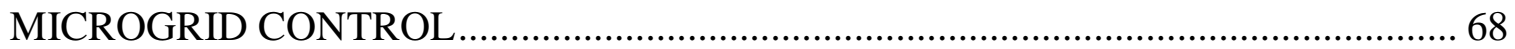

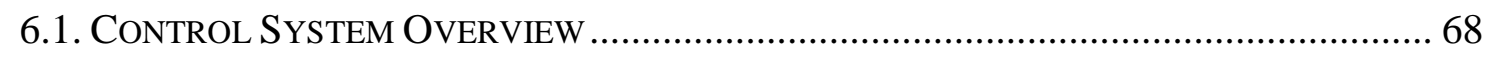

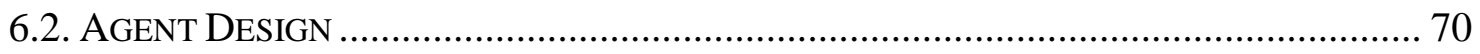

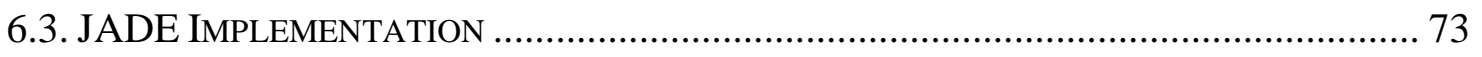

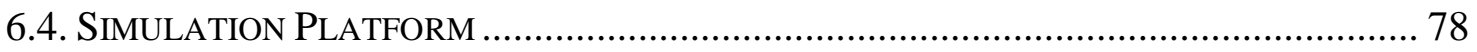

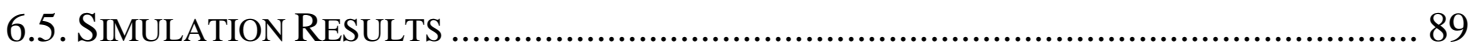

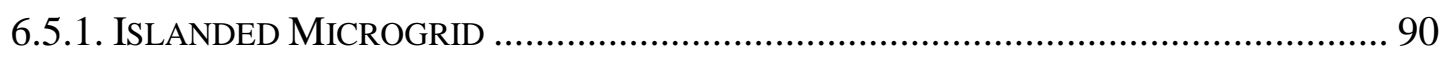

6.5.2. GRID CONNECTED MG .............................................................................. 94

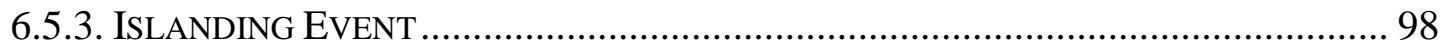

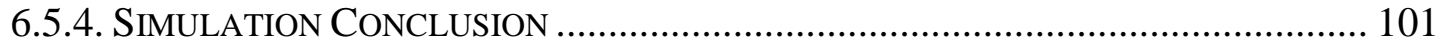

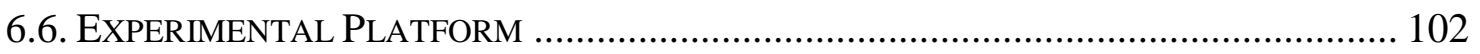

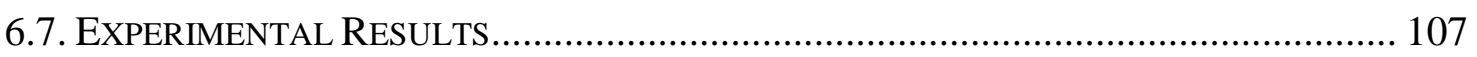


7. CONCLUSION AND RECOMMENDATION FOR FUTURE WORK

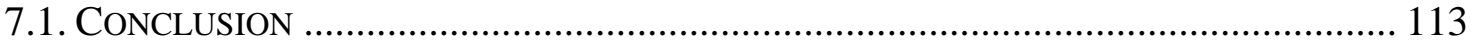

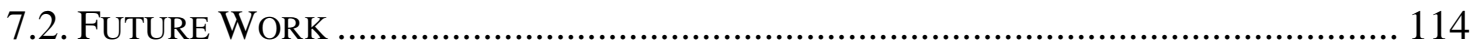

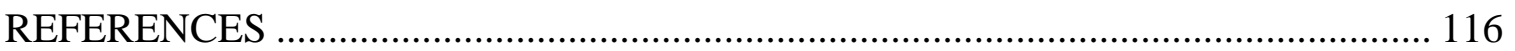

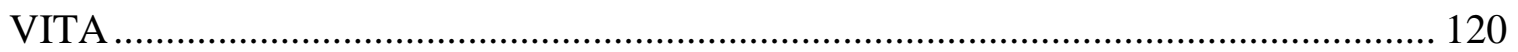




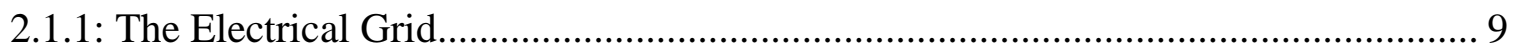

2.3.1: Hypothetical 7-Node Microgrid........................................................................... 12

3.1.1: Typical Fossil Fuel Generator Cost Function ........................................................ 18

3.1.2 Typical Fossil Fuel Marginal Cost Function ............................................................ 18

3.2.1: Automatic Generation Control .......................................................................... 21

3.3.1: Isochronous Control .................................................................................... 22

3.3.2: Speed-Droop Characteristic ………………………………………………..... 23

3.3.3: Speed-Droop Characteristic, Two Generators ………………………………... 24

3.3.4: Droop Control ............................................................................................... 25

3.3.5: Adjusting No-Load Frequency …………………………………………….... 26

4.2.1: Graph Example .............................................................................................. 33

4.3.1: Energy Storage System Cost Function …………................................................... 38

4.3.2: Energy Storage System Marginal Cost Function..................................................... 39

4.3.3: Energy Storage System Cost Function Surface ……………..................................... 39

4.3.4: Energy Storage System Marginal Cost Function Surface ……………………....... 40

4.3.5: Microgrid MAS Agent Assignments ......................................................................... 41

5.1.1: MG Network Node Layout .............................................................................. 46

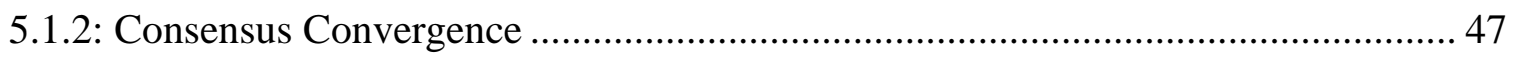

5.2.1: Diffusion Convergence …………………………................................................ 49

5.4.1: Two-Step Diffusion Methodology …………………………………………….... 56

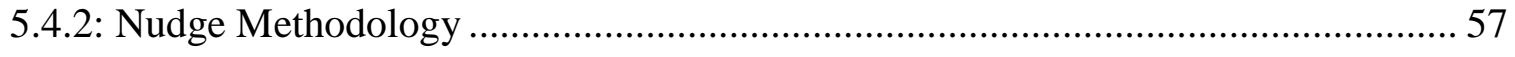

5.4.3: Islanded MG Cost Functions ED ....................................................................... 58 
5.4.4: Islanded MG Marginal Cost Functions ED ........................................................ 59

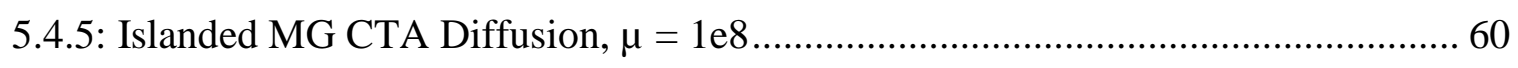

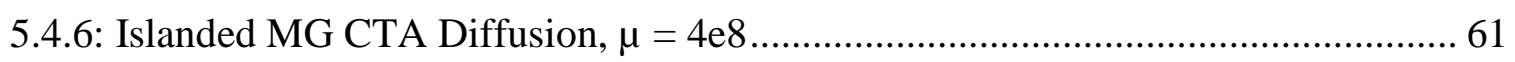

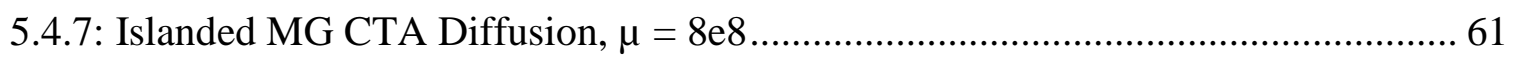

5.4.8: Grid-Connected MG CTA Diffusion, $\mu=1$ e8 .................................................... 63

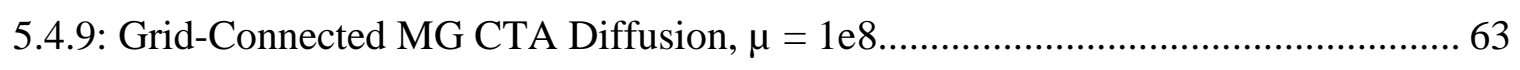

5.4.10: Fossil Fuel Generator Cost Function with Constraint Penalties ............................. 65

5.4.11: Fossil Fuel Generator Marginal Cost Function with Constraint Penalties ............ 65

5.4.12: Grid-Connected MG CTA Diffusion w/Constraints, $\mu=1 \mathrm{e} 8$.................................. 67

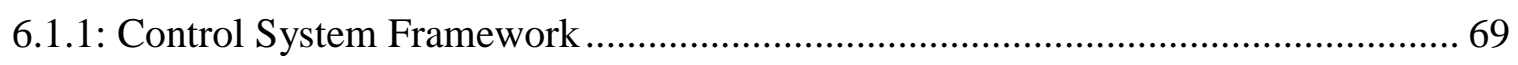

6.2.1: Stochastic Agent Block Diagram......................................................................... 71

6.2.2: Dispatchable Agent Block Diagram .................................................................... 72

6.3.1: Multi-Agent System Java Implementation using JADE........................................... 74

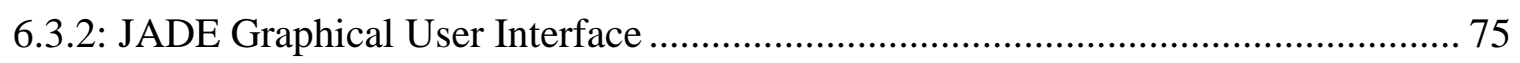

6.3.3: JADE Sniffer, Initial SUBSCRIBE and INFORM messages.................................. 76

6.3.4: JADE Sniffer, PROPOSE message on IS convergence............................................ 77

6.4.1: Hypothetical MG for Simulation ...................................................................... 79

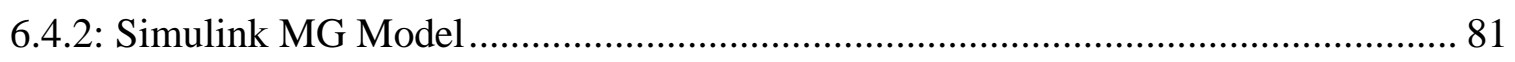

6.4.3: Simulink Grid Subsystem ............................................................................... 82

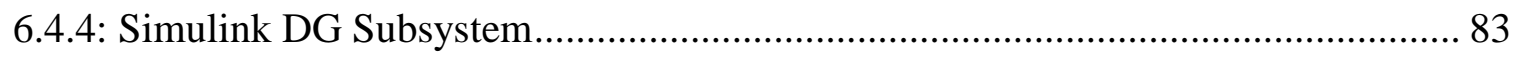

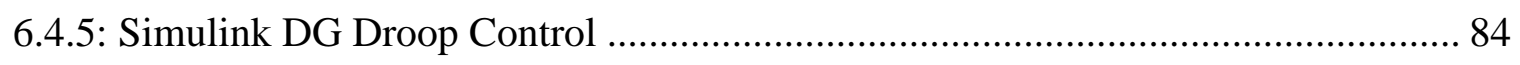

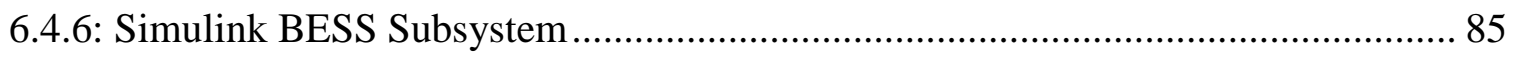

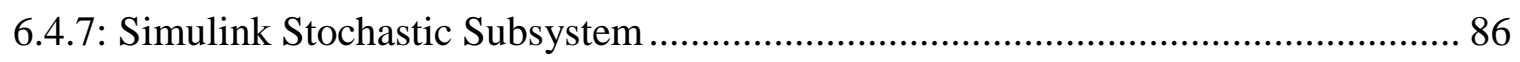

6.4.8: Commercial Load Profile.................................................................................... 86 


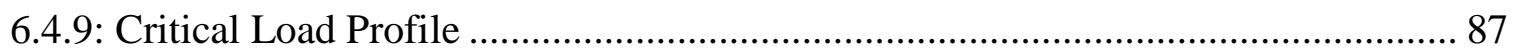

6.4.10: Residential Load Profile .................................................................................... 87

6.4.11: Inerjy EcoVert 250 Output Power vs Windspeed.............................................. 88

6.4.12: Wind Turbine Generation Profile for the city of Key Biscayne (3/20/2009 @

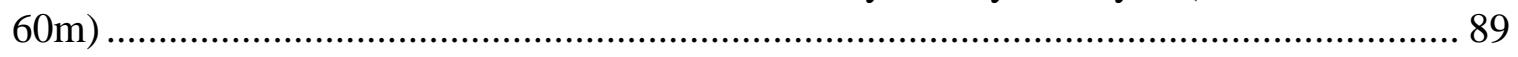

6.4.13: Solar PV Generation Profile for the city of Key Biscayne (3/20/2009) ................ 89

6.5.1: MG Net Power Demand, Actual vs Diffusion............................................................ 90

6.5.2: MG Net Power Demand, Actual vs Diffusion, Close-up ......................................... 91

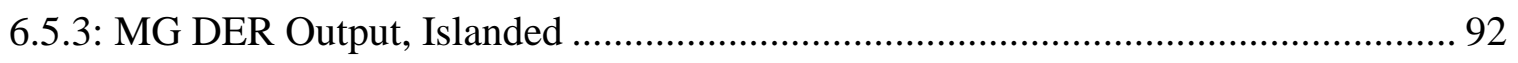

6.5.4: DER No-Load Frequency Adjustments, Islanded .................................................... 92

6.5.5: DG No-Load Frequency Adjustments, Islanded, Close-up ....................................... 93

6.5.6: BESS Power Output vs SOC, Islanded..................................................................... 93

6.5.7: MG Frequency and Voltage, Islanded .................................................................... 94

6.5.8: Electric Utility TOU Rate Structure …………………………………………........ 95

6.5.9: MG DER Output, Grid-Connected ...................................................................... 95

6.5.10: DER No-Load Frequency Adjustments, Grid-Connected ...................................... 96

6.5.11: DER No-Load Frequency Adjustments, Grid-Connected, Close-Up.................... 97

6.5.12: BESS Power Output vs SOC, Grid-Connected ………………….......................... 97

6.5.13: MG Frequency and Voltage, Grid-Connected....................................................... 98

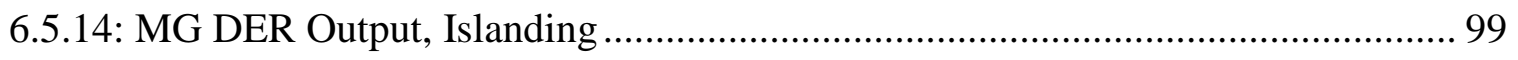

6.5.15: BESS Power Output vs SOC, Islanding ……………………………………...... 99

6.5.16: MG Frequency and Voltage, Islanding ............................................................ 100

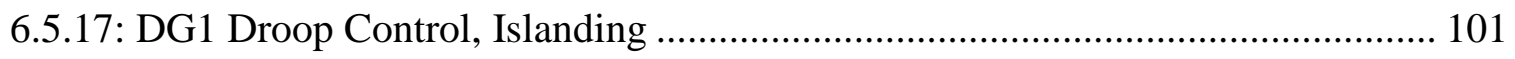

6.6.1: Smart Grid Testbed, Alternating Current Side ..................................................... 102

6.6.2: Smart Grid Testbed, Direct Current Side........................................................... 103 


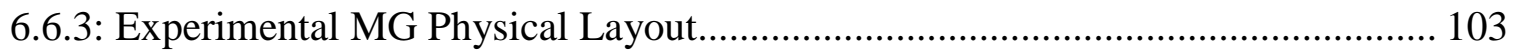

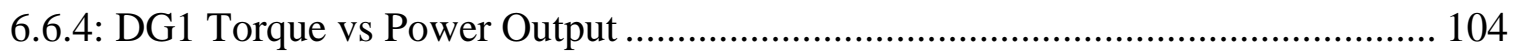

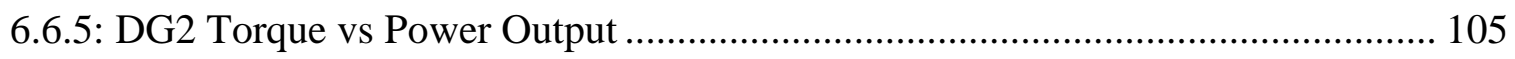

6.6.6: Experimental MG Network Topology ........................................................ 106

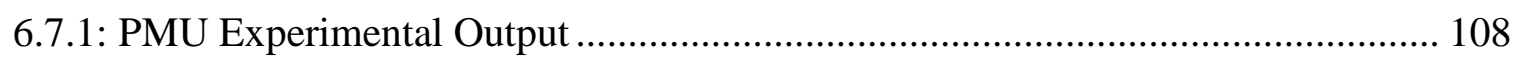

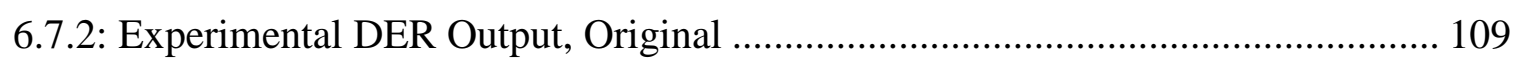

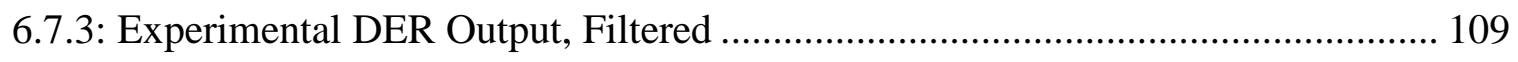

6.7.4: Experimental DER Output vs Frequency, Close-Up ....................................... 110

6.7.5: Experimental DER Output vs Frequency, Zoom-Out ...................................... 111

6.7.6: Experimental System Voltage and Frequency.............................................. 112 


\section{CHAPTER 1: INTRODUCTION}

\subsection{RATIONALE}

The concept of the Microgrid (MG) has been gaining traction as a major piece of the puzzle in the evolution towards a reduced carbon footprint and the development of the Smart Grid. By creating smaller networks typically at Low or Medium Voltages (LV, MV), MGs can utilize the waste heat from Distributed Generation (DG) sources for space heating and cooling as well as industrial processes. These Combined Heat and Power (CHP) systems greatly improve the energy efficiency and therefore reduce emissions. Also, MGs aim to integrate Renewable Energy Sources (RES) along with Energy Storage Systems (ESS) to further reduce their $\mathrm{CO}_{2}$ emissions and help meet environmental mandates [1], [2]. MGs are semi-autonomous entities while connected to the grid, when they can either buy electricity from, or sell excess generation to the operating utility. When not connected to the grid, they are said to be operating in islanded mode where the balance of supply and demand is entirely established within the confines of the MG network. The latter situation presents a challenge, especially with a large percentage of RES within the system because of the stochastic nature of these resources, the lack of inertia for inverter-based energy sources and the low inertia inherent to small DG sources.

Low inertia in an Alternating Current (AC) system means that disturbances in the generation and load balance will have relatively larger effects on the system frequency and voltage, which may cause stability issues and even blackouts. In MGs, this problem is mitigated by having fast-acting ESS, such as banks of batteries (BESS), which can quickly inject or absorb energy into or from the system. Additionally, droop control has been proposed as the best solution for decentralized primary control of inverter-based MGs, as 
they can emulate virtual inertia. Voltage Source Inverters (VSI) with droop control, which can be subsequently adjusted through secondary control after each imbalance event, have been proposed [3]. A similar droop control method for primary control is explained and implemented, as well as a decentralized Multi-Agent System (MAS) for secondary and tertiary control, which will be described next.

Control of system frequency and stability is categorized as primary control, which is automatic and component-centric. Secondary control adjusts the operating conditions after an imbalance with a global system perspective. The owner of a MG also looks to operate it as efficiently as possible to reduce costs, losses and/or emissions in the system; this is classified as tertiary control. ESS introduce another interesting optimizable component, as they have the ability to store inexpensive electricity (say at night when utility prices are low or during high RES outputs) and utilize it in peak demand times (when utility prices are at their highest or during low RES outputs); this is referred to as Energy Arbitrage (EA).

MAS are the focus of much ongoing research, as they present a very flexible and robust framework for distributed systems [4] [5]. In a MAS, each agent is intelligent and independent and has the ability to communicate and cooperate with other agents for a common goal. For MGs, this concept introduces an exciting possibility of having a reliable and adaptable electrical network able to grow and evolve without the need for much infrastructure and centralized control. In this work, a MAS is implemented where each DG, ESS and load feeder is represented by an agent that communicates only with adjacent agents, yet they all seek a global objective. 
This idea of decentralized communication is another subject of considerable research. Sayed et al. [5] [6] [7] [8] have developed the Diffusion Algorithm (DA), which includes an additional gradient term when compared to the consensus strategy in order to “diffuse," or spread, the information much more quickly. Furthermore, the DA is more sophisticated than consensus in that it can also perform distributed optimization without the need of a centralized aggregator. In a MG, DA communication can be used to quickly disseminate new operating conditions and reach a global optimum for ED in a completely decentralized manner; this has never been proposed before in the context of MG control.

A comprehensive MG operation and control scheme, which joins droop control for primary, and a MAS using DA communication for secondary and tertiary control, is presented. The MAS architecture will follow the Foundation for Intelligent Physical Agents (FIPA) standards and will be implemented using the Java Agent Development Framework (JADE). Simulations will be realized on Matlab Simulink software and real-time experimental results will be obtained in a Smart Grid Testbed [9], [10].

\subsection{LITERATURE REVIEW}

The latest literature in terms of MG resource optimization includes [11] where the authors implement Optimal Power Flow (OPF) considering system constraints and Local Controllers coordinate with the Microgrid Central Controller to reach each resource's optimal power point. In paper [12] a Model Predictive Control which uses Support Vector Machines to forecast RES output and load levels is combined with Mixed Integer Linear Programming to minimize the MG operating costs. A Multi-Objective Genetic Algorithm using high temporal resolution insolation data is used to find the optimal design of a DC 
MG in [13]. These optimization methods so far depend on a central controller and are thus vulnerable to the single point of failure (SPOF) and the curse of dimensionality problems given the complicated communication infrastructure and high computational demand of the central entity as more elements are added to the MG.

The most promising alternative to central control schemes are Multi Agent Systems which involve intelligent entities with the ability to control themselves and communicate with each other. Paper [14] proposes a MAS architecture using Replicator Dynamic Theory to reach the economic operating point of a MG, however a "Dominant Agent" is used to aggregate the cost functions of the other agents and thus defeats the purpose of decentralization. A good framework for MG MAS is presented in [15], but the optimization and communication among agents is not detailed.

The latest literature in terms of distributed primary and secondary MG control includes [16], where the authors implement a decentralized MAS (DMAS) architecture to control the various RES power points to maintain the system frequency with the aid of droop-controlled DGs. Paper [17] uses a consensus strategy to adjust the DGs and Load Shedding to maintain the system frequency. In [18] a network of dynamic fuzzy agents control the MG voltage through distributed consensus. However these papers focus only on the primary and secondary control of the energy resources and do not consider the optimal operation or Economic Dispatch (ED) of them.

Considering tertiary control, in [19], a droop controlled DC MG is optimized by a DMAS using the Dynamic Consensus Algorithm, though only the global efficiency of the converters is considered. A similar but modified consensus algorithm is used in [20] for optimal resource management in a MG, but the primary control is not detailed. Another 
revision on the consensus algorithm is used in [21] plus a multistep optimization which considers ESS for EA, however the primary control is again not mentioned.

\subsection{ORIGinal CONTRIBUtions}

To the best of the author's knowledge, there hasn't been an all-inclusive decentralized MG control structure to robustly manage primary, secondary and tertiary control like the one proposed in this thesis. Furthermore, the diffusion algorithm that greatly improves on the standard consensus methodology has never been implemented in the context of Microgrids or power systems. Additionally, distributed optimization using the diffusion strategy not only improves previous decentralized economic dispatch attempts, but combines secondary and tertiary control in an elegant fashion.

Also, an innovative technology-agnostic cost function for an ESS is presented, which automatically charges when the rate of electricity (or marginal cost) is low and discharges when high. Perhaps some of the most significant contributions of this thesis are the experimental setup and results. Such a comprehensive decentralized control system has never before been experimentally tested in the literature to the best of the author's knowledge.

\subsection{THESIS OVERVIEW}

The rest of this thesis is organized as follows:

- Chapter 2 provides a brief introduction on the current electrical grid infrastructure and why and how it has evolved to its current state. Then, Smart Grid technology is defined 
and the concept of the Microgrid and its role is described. Finally, the difference between a grid-tied and islanded Microgrid is explained as a segue into the next chapter.

- Chapter 3 is focused on the basics of power systems including economic dispatch and generator control systems. Additionally, droop control is described and its importance for Microgrids is explained.

- Chapter 4 provides a comparison between centralized and decentralized control systems to provide the motivation behind the proposed distributed MG control framework. Then, network and dynamic systems basics are introduced, and the concept of Multi-Agent Systems is described.

- Chapter 5 provides the background on adaptive networks and dives into the diffusion algorithm, and compares it to similar strategies like consensus. Decentralized optimization using diffusion is then explained, and its application to MG economic dispatch is presented.

- Chapter 6 puts all previously mentioned concepts together into a complete MG control system that is fully decentralized, adaptive, automated and resilient. Each component is fully explained and the simulation and experimental setup and results are presented.

- Chapter 7 concludes the work and suggests possible areas of further research. 


\section{CHAPTER 2: MICROGRIDS}

This chapter will describe how the electric grid was formed and why it is considered to be outdated. Then, Smart Grid technology will be introduced to explain the future outlook of power systems and Microgrids to clarify the reason for developing an effort on this topic of research. The challenge of islanded power systems will be defined to give background on the type of problems that need to be solved.

\subsection{THE FORMATION OF THE ELECTRIC GRID}

The history of the electrical grid dates back to the late nineteenth century, when developments of applications for the electrical and magnetic discoveries of earlier in the century were starting to arise. In the United States, two technologies were competing for dominance of the electrical market, Direct Current (DC) and Alternating Current (AC). Thomas Edison, who championed DC, had developed the incandescent light bulb and was building DC power stations around Manhattan to sell power and light bulbs to the people of New York. On the other hand, George Westinghouse had licensed Nikola Tesla’s AC patents and won a contract to build a hydroelectric power plant in Niagara Falls [22].

Edison pushed hard to gain dominance in the industry, citing the advantages of DC like flicker-free light and simple DC motor control, and even launched a smear campaign against AC, claiming it was more dangerous. However, the main advantage of AC, which was the ability to transform the voltage, ultimately tipped the scale in its favor. This was so important because by doubling the voltage the amount of current needed is halved in order to transmit the same amount of power; this in turn reduces the losses due to resistance by a factor of four. 
Edison argued that this could be solved by having smaller generators close to the loads in order to reduce the resistance. However, due to the economies of scale of everlarger power plants and high-voltage transmission lines, AC was the clear champion. Large generation stations were placed where topography permitted and where economically advantageous. Large hydro-electric plants were obviously located wherever there was the possibility, and nuclear plants were eventually placed where deemed safe and with ample amounts of water for cooling. Fossil fuel plants were positioned close to mines, wells and railroads and as far away from population centers as possible due to their polluting nature.

Samuel Insull was another key player in the development of the grid; he vastly accelerated the adoption of electrification in order to spread the great capital costs among a larger consumer base. He also developed the financial and regulatory structures still present today, such as selling common stock of the utilities and setting up Public Utility Commissions. Regulated monopolies were established per region in order to avoid having separate transmission and distribution power lines per utility company for the same customer base.

In essence, much hasn’t changed for over 100 years; as shown in Fig. 2.1.1, the basic structure of centralized power generation with transmission and distribution lines conceived during that time still exists today.

It wasn't until the second half of the twentieth century that the development of semiconductors and power electronics allowed for DC transformation. Nowadays, electrical power can easily be converted from AC to DC and vice-versa. Many cases actually exist of High-Voltage DC (HVDC) connections to transfer power between separate AC grids. Long-distance HVDC transmission lines are also possible and in fact they have 


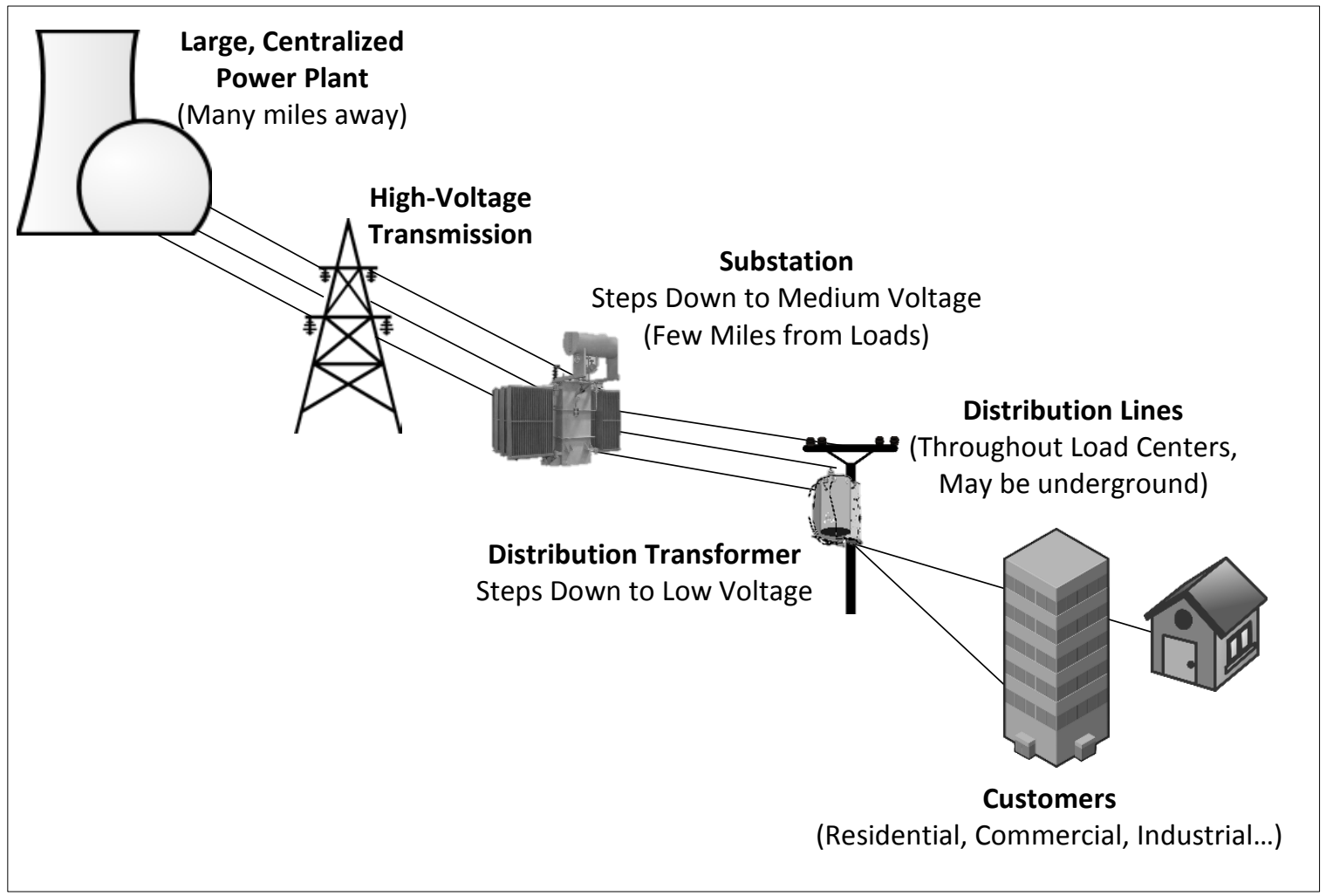

Figure 2.1.1: The Electrical Grid

less losses than their AC equivalents, but they remain uncommon and are still less costeffective (even though the costs are decreasing).

The fact of the matter is that in most developed countries there exists a vast AC infrastructure that is aging and in need of modern technology to cope with the latest trends. In the next sections, these trends will be explained and the latest technologies and concepts will be introduced.

\subsection{SMART GRID TECHNOLOGY}

In the past decades, the revolution brought about by exponential technological advancement has created a real push towards the modernization of the grid. "Smart Grid" refers to the conceptualized vision of the evolving electrical grid in adapting the latest 
computing, automation and communication developments [23]. Because electricity is such a valuable and essential resource in today's world, the progress has been slow and has not kept up with other industries, but the trend is clear.

New grid technologies include the incorporation of more and more sensors like Phasor Measurement Units (PMUs or synchrophasors), which provide real-time, GPSsynchronized data to monitor the current status of the electrical system across large distances. Smart meters on every home and business are being widely adopted and provide unprecedented amounts of data in order to more accurately forecast future energy demands. Smart meters also allow two-way communication and stimulate "Smart Home" technologies, where, for example home appliances can react to price signals, which could schedule energy-intensive tasks during lower-rate periods. Utility signals are also being used more and more to remotely shut off non-critical loads for what is termed Demand Response, mainly used when the grid is overloaded.

Reliability is a major concern for electric utilities, and new "smart" equipment on the grid can react to emergencies automatically and re-route the power distribution to make outages shorter and less frequent. Energy Storage is another industry that is accelerating rapidly and receiving much-needed attention. Currently, storage systems are mainly being used for voltage and frequency regulation, and we are now seeing them replace expensive natural gas "peaker" plants as they are assets that have many value streams and are "always on”. Whereas peaker plants operate at a small fraction of their Capacity Factor (or yearly production potential) and have very high marginal costs, energy storage facilities can provide almost instantaneous support for the grid all year round and additionally perform 
Load Shifting: storing energy during low-demand periods to provide it during high-demand periods.

The slow but growing adoption of electrical vehicles also poses a challenge and great opportunity for the Smart Grid. Electric vehicles present significantly large and dynamically-located energy loads which have the potential of introducing heavy stress to the power system. On the other hand, they can also be seen as wide-spread energy storage assets, which could provide extremely valuable services to the grid and/or the owners of the vehicles. By integrating intelligent systems into the management of the charging and discharging of the vehicles, they can be leveraged while maintaining the transportation requirements of the user [24].

All these technologies plus advanced control systems are making the grid more efficient and reliable, and are allowing more and more penetration of intermittent Renewable Energy Sources (RES). As regulations become stricter regarding emissions of greenhouse gasses, the adoption of RES will continue to accelerate. The stochastic nature of these energy resources presents a challenge to the grid, as the constant balance of supply and demand needs to be strictly maintained in order to have a stable system. These Smart Grid technologies and further breakthroughs will be needed in order to accomplish the decarbonized electrical system of the future.

\subsection{THE CONCEPT OF MICROgRIDS}

The term Microgrid (MG) first appears in the literature in 2001, being proposed as a semi-autonomous cluster of Distributed Generation (DG) sources, Energy Storage Systems (ESS) and loads operating as a single controllable system. They are meant to 
enhance the efficiency and reliability and reduce the operating costs of the customer while potentially providing services to the grid. They also present the possibility of decentralizing the grid by creating distributed intelligent energy cells across the power system that can theoretically communicate with each other and/or with a central controller to achieve further efficiency and reliability [25], [26].

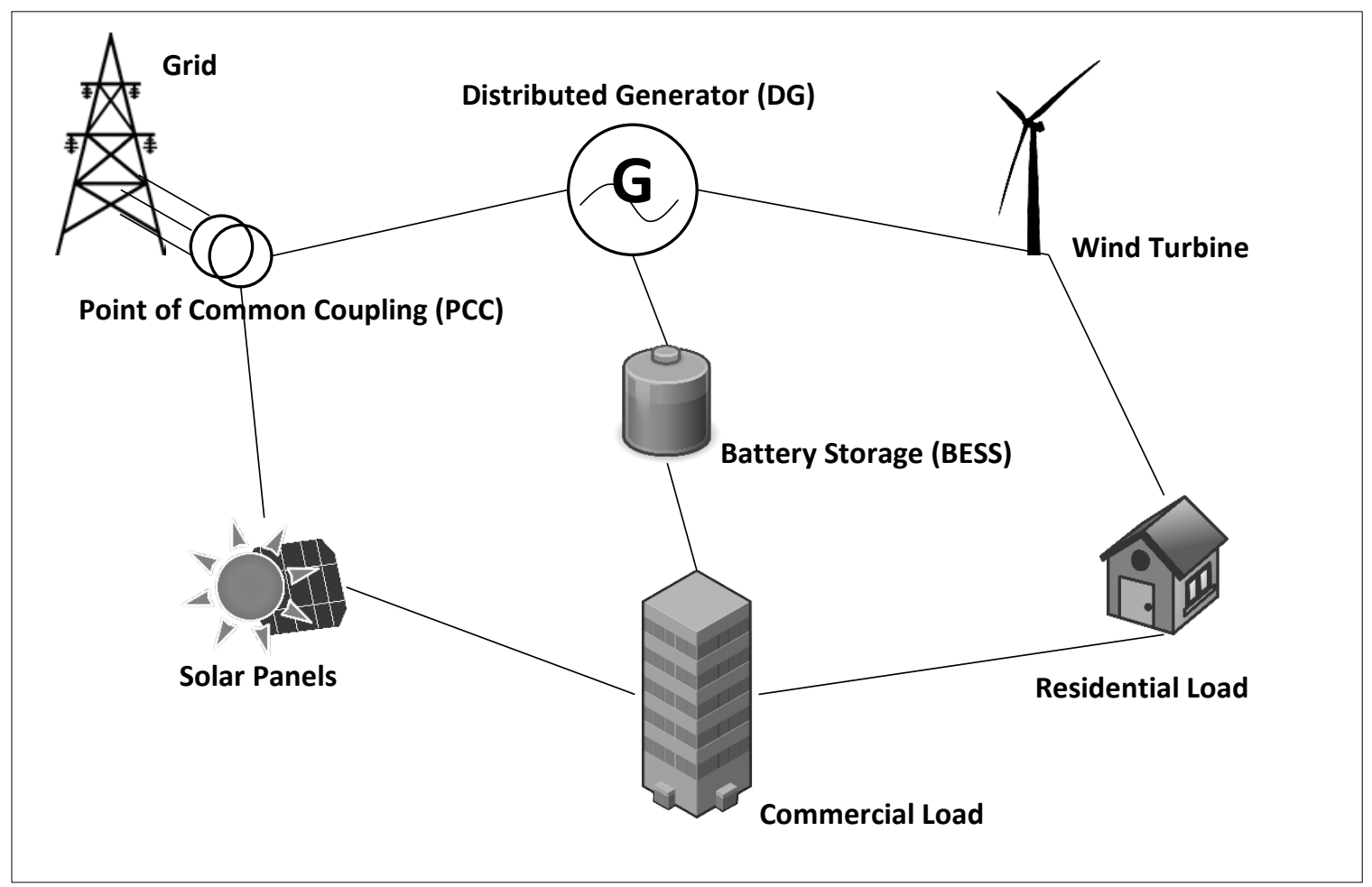

Figure 2.3.1: Hypothetical 7-Node Microgrid

As the term implies, Microgrids are smaller electrical networks typically at Low or Medium Voltages (LV, MV), as seen in Fig. 2.3.1. By reducing the distance between sources and loads, MGs diminish losses associated with transmission. They also aim to utilize the waste heat from Distributed Generation (DG) sources for space heating and cooling, as well as for industrial processes. These Combined Heat and Power (CHP) systems greatly improve energy utilization and efficiency. Fast-acting and automated 
communication and control systems allow MGs to integrate large amounts of Renewable Energy Sources (RES) and maintain system stability (which is the main purpose of this thesis).

ESS are typically part of a MG to provide greater resiliency and reliability akin to an Uninterruptible Power Supply (UPS). They also help in the integration of RES and additionally offer the ability to perform Energy Arbitrage (EA): charging during low-rate periods and discharging during high-rate periods to reduce energy costs. ESS are even more important when the MG is not in the vicinity of the main grid or gets sectionalized for whatever reason, as is explained in the next section.

Most current-day examples of MGs are seen on university campuses and military facilities; however, they have the potential to transform the electrical power system. With more and more Distributed Energy Resources (DERs) being installed on the grid, the ability to manage them all in a reliable and efficient way becomes an increasingly complex problem. MGs offer the capability of administering these DERs in semi-autonomous cells, each one responsible for its own system stability and able to provide "assistance" to neighboring cells or the grid as a whole. Although still in the works, novel regulations and standardized communication protocols could allow MGs to participate in wholesale markets and effectively aid in creating a more stable, efficient and economical power system [1].

MGs are not yet widespread, and the technology can still be considered in its early stages (thus the interest of the author). They are still not economically competitive due to the highly-tailored nature of their control systems and the high capital costs of their 
components, especially ESS. The next section covers one of the great challenges in MG research, the islanded power system.

\subsection{ISLANDED POWER SYSTEMS}

When Microgrids (MG) are grid-connected, their AC frequency and phase are synchronized with the utility. Because the grid is so much larger, it is considered to have infinite inertia, meaning a change in any given load will not affect the system frequency in a significant way, as explained shortly. In this operating scenario, the MG buys or sells electricity to the utility according to the rate being charged or offered by the market, and the MG's Energy Storage System (ESS) may engage in Energy Arbitrage (EA), as explained in the previous section. In the event of a supply-demand imbalance caused by a large load or source being connected or disconnected, the extra energy would be immediately supplied or absorbed mostly by the grid without much trouble.

When not connected to the grid, MGs must maintain the balance of supply and demand without the inertia offered by the grid; this is deemed an "islanded" power system. This operating scenario presents a challenge due to the low inertia inherent to small DG sources and lack thereof for inverter-based energy sources (such as renewables). Low inertia in an AC system means that disturbances in the generation and load balance will have relatively larger effects on the system frequency and voltage, which may cause stability issues and even blackouts.

This is analogous to riding a bike; when pedaling at a constant speed on a flat road the bike neither accelerates nor decelerates. If the rider maintains the same power to the pedals when going uphill, the bike will undoubtedly slow down, or vice-versa when going 
downhill. In this comparison, the rider is the generator, the speed of the bike is the frequency of the power system, and the slope is the amount of load.

The relationship between inertia and frequency is given by the swing equation [27]:

$$
\frac{2 H}{\omega_{s y n}} \frac{d^{2} \delta}{d t^{2}}=P_{m}-P_{e}=P_{a}
$$

Where $H$ is the normalized inertia constant, $\omega_{\text {syn }}$ is the system's angular velocity (synchronous frequency), $d^{2} \delta / d t^{2}$ is the angular acceleration, and $P_{a}$ is the accelerating power, which equals the mechanical minus the electrical power. Rearranging for angular acceleration:

$$
\frac{d^{2} \delta}{d t^{2}}=P_{a} \frac{\omega_{s y n}}{2 H}
$$

Thus, for the same power imbalance $\left(P_{a}\right)$, a smaller inertia $(H)$ means a larger angular acceleration, which translates into a quicker deviation of the system frequency.

Renewable Energy Sources (RES) add another layer of complexity due to the stochastic and intermittent nature of these resources. With larger amounts of RES within a MG, the system must balance generation with stochastic load profiles and larger output swings from these sources. ESS become crucial in this scenario to provide this balance, as they are typically very fast-acting like in the case of large Battery systems (BESS).

In the next chapter, the background necessary to understand power system control and optimization will be provided. Also, droop control will be presented as a major tool for low-inertia system stability. , 


\section{CHAPTER 3: POWER SYSTEM OPTIMIZATION AND DROOP CONTROL}

In this chapter, the basics of Economic Dispatch are given in terms of optimization of smaller power systems such as Microgrids (MG). This will allow control systems to be briefly outlined in the context of power system stability and economic operation. Droop control and how it can be applied to Microgrids will then be described.

\subsection{INTRODUCTION TO ECONOMIC DISPATCH}

The problem of Economic Dispatch (ED) is one of optimization; at what power output level should dispatchable assets be operated to meet demand in the most costeffective manner? Mathematically, the problem can be described as a set of cost functions for each generator, with a set of minimum and maximum power levels for each, (inequality constraints), and an equality constraint describing that the total power of all the generators must be equal to the total load [28]:

$$
\begin{gathered}
F_{T}=\sum_{i=1}^{N_{\text {gen }}} F_{i}\left(P_{i}\right) \\
P_{i, \text { min }} \leq P_{i} \leq P_{i, \max } \\
P_{\text {load }}=\sum_{i=1}^{N_{\text {gen }}} P_{i}
\end{gathered}
$$

Where $P_{i}$ is the $i^{\text {th }}$ generator's power output, $F_{i}\left(P_{i}\right)$ is the cost function of that generator for any given power level, $P_{i, \min }$ and $P_{i, \max }$ are the minimum and maximum power levels of that generator, respectively, $N_{\text {gen }}$ is the total number of generators in the system, $P_{\text {load }}$ is the total load power demand and $F_{T}$ is the total cost of the generation scheme. The 
objective is to minimize $F_{T}$ while maintaining all inequality and equality constraints (ignoring losses).

Generator cost functions are usually non-decreasing, meaning the cost increases as the power increases. Furthermore, fossil-fuel generators are typically simplified into a quadratic cost function, which is non-decreasing and twice-differentiable; in this section, we will focus on such generators since they are the most characteristic DG sources:

$$
\begin{gathered}
F_{f f}=a+b P_{f f}+c P_{f f}^{2} \\
\frac{d F_{f f}}{d P_{f f}}=b+2 c P_{f f} \\
P_{f f, \text { min }} \leq P_{f f} \leq P_{f f, \text { max }}
\end{gathered}
$$

Where $F_{f f}$ is the quadratic cost function for a fossil fuel plant, $a, b$, and $c$ are the quadratic coefficients, $P_{f f}$ is the power level for that unit, and $d F_{f f} / d P_{f f}$ is the derivative of the cost function with respect to power. As can be seen in Fig. 3.1.1 and 3.1.2, as the power output increases, not only does the cost increase, but the marginal cost (or incremental cost of the next watt) also increases.

For such cost functions, there exists a global minimum for the optimization objective of minimizing the cost, meaning there is one unique combination of generator power levels that yields the most cost-effective solution. The Lagrange method is used to find this point and reaches the conclusion of having all units' incremental costs equal to each other (as long as they are within their limits):

$$
\frac{d F_{i}}{d P_{i}}=\lambda
$$




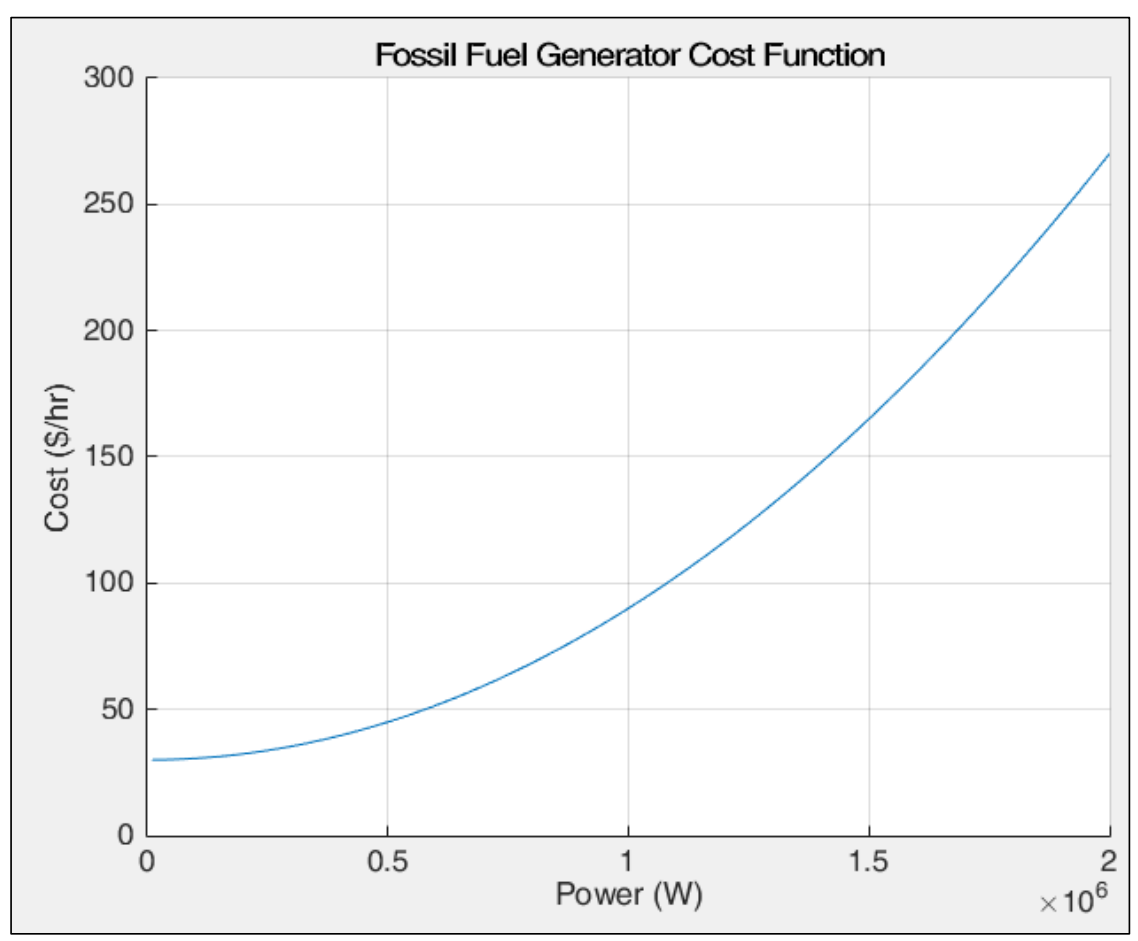

Figure 3.1.1: Typical Fossil Fuel Generator Cost Function

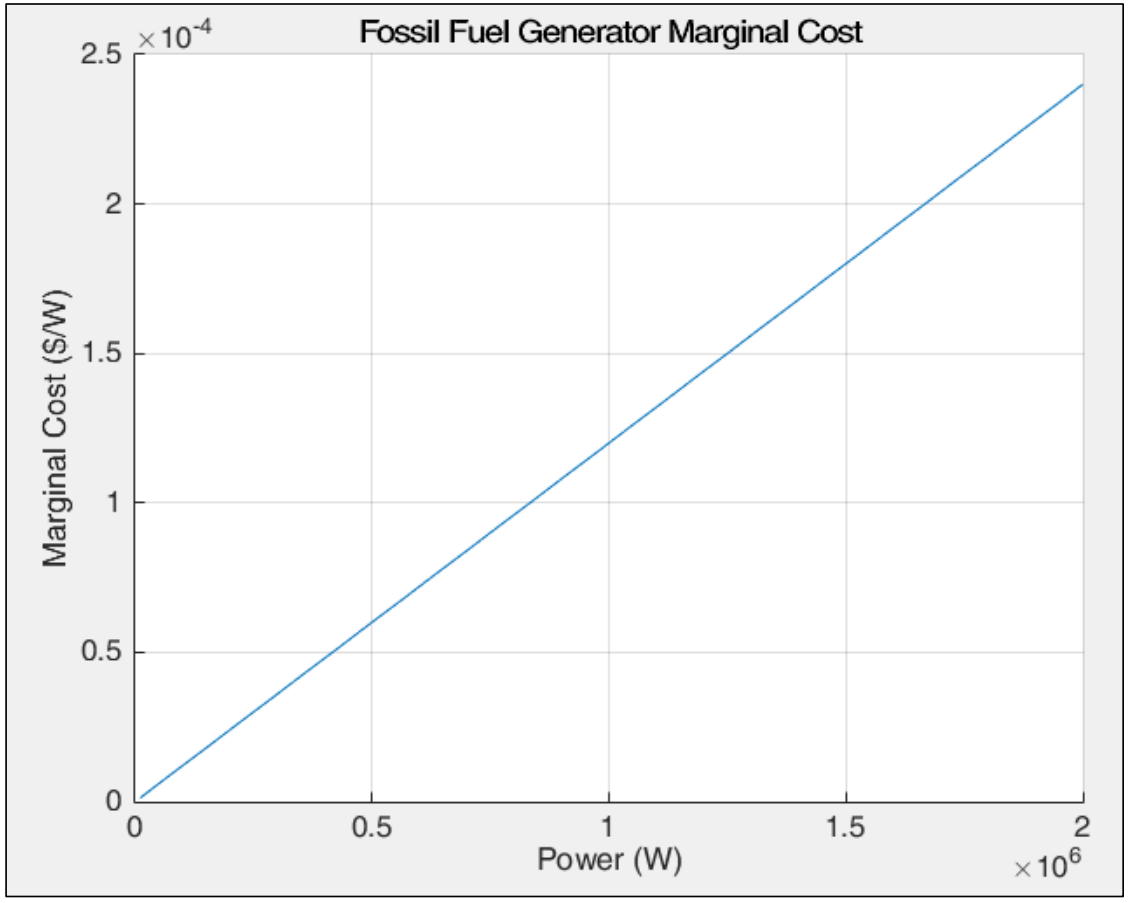

Figure 3.1.2 Typical Fossil Fuel Marginal Cost Function 
Where $d F_{i} / d P_{i}$ is the derivative of the cost function for the $i^{\text {th }}$ unit with respect to power (also known as the incremental or marginal cost) and $\lambda$ is the Lagrange multiplier. This equation describes what was mentioned previously; the global minimum is reached when each generator's marginal cost is equivalent. Intuitively, if one generator's marginal cost is greater than the others, then by decreasing that unit's power level and increasing the others by the same amount collectively, a lower total cost will be reached since the former unit "stepped down" in cost a greater amount than the others "stepped up". This concept will be key when optimization of Microgrid resources is revisited in later chapters.

Microgrids are essentially a mini-power system where the same concept of ED applies on a smaller scale. Because of the proximity of MG components, the assumption will be made that losses are kept to a minimum and can therefore be ignored. It will be seen in Chapter 6 how this optimization is done in a decentralized fashion.

\subsection{INTRODUCTION TO GENERATOR CONTROL SYSTEMS}

As a large and complex system, the electric grid utilizes automatic control schemes to maintain system stability. As described in Section 2.4, the balance between generation and load must be maintained at all times, and because the system is AC, frequency is mainly used to measure this balance. The swing equation (eq. 2.4.1 and 2.4.2) describes how a change in the power balance will affect the angular acceleration in relation to the system inertia. The angular acceleration is equal to change in frequency:

$$
\frac{d^{2} \delta}{d t^{2}}=\frac{d \omega}{d t}
$$


Therefore, the difference between $P_{\text {mech }}$ and $P_{\text {elec }}$ needs to be corrected as quickly as possible; this is done by the Automatic Generation Control (AGC). AGC not only keeps the system frequency stable, but it also accounts for tie-line flows which have been negotiated with adjacent control areas; together they are known as the Area Control Error (ACE). Furthermore, AGC maintains each generator operating at its ED point, and keeps track of total generation across the control area. This control scheme is described in Fig. 3.2.1.

The tie-line power flow is related to ED in that a control area with a higher marginal cost than an adjacent area will choose to "import" that power from its neighbor, if possible, to reduce costs. These contracts are negotiated hours and days in advance, but if there is an imbalance in a given control area, the power flow will be affected not only within the area, but also through the tie-line. Therefore, the tie-line flow needs to be constantly monitored and the generation adjusted to maintain the contracted agreements.

As described in [28], AGC has three major objectives:

1. To hold system frequency at the nominal level (60 $\mathrm{Hz}$ in the USA).

2. To maintain the correct tie-line power between control areas.

3. To maintain each unit at its Economic Dispatch level.

However, one more tool is used in order to maintain transient stability. This is droop control, as explained in the next section. 


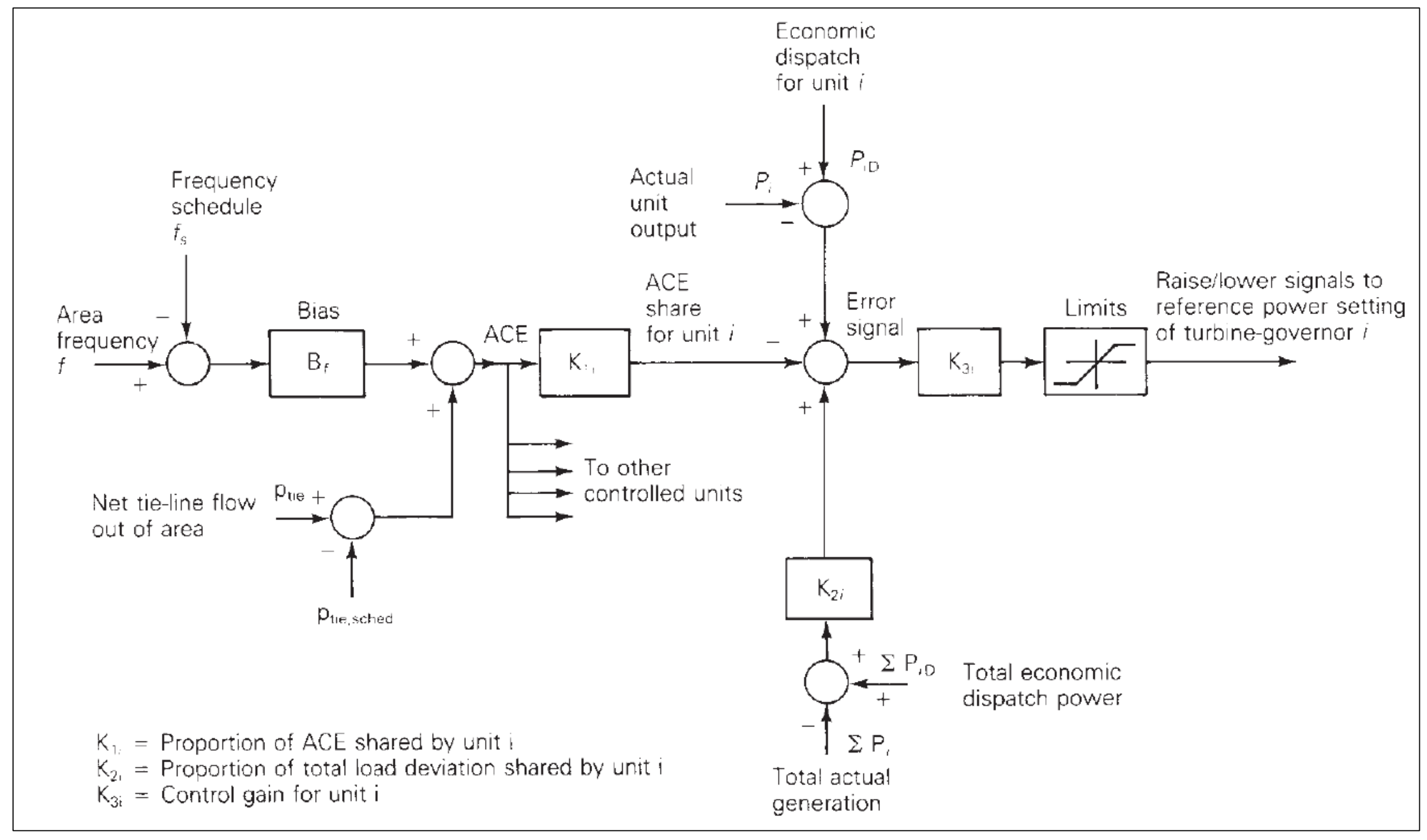

Figure 3.2.1: Automatic Generation Control [27] 


\subsection{DROOP CONTROL BASICS}

When there is only one generator working in an islanded power system, it can be set to maintain the frequency at a nominal value regardless of the load. Ignoring transients, the generator control will adjust the mechanical power to match the electrical load; this is called isochronous operation. Fig. 3.3.1 describes the system used to implement this mode of operation, where the speed error is integrated and the mechanical power is adjusted according to the deviation from the reference frequency [28]:

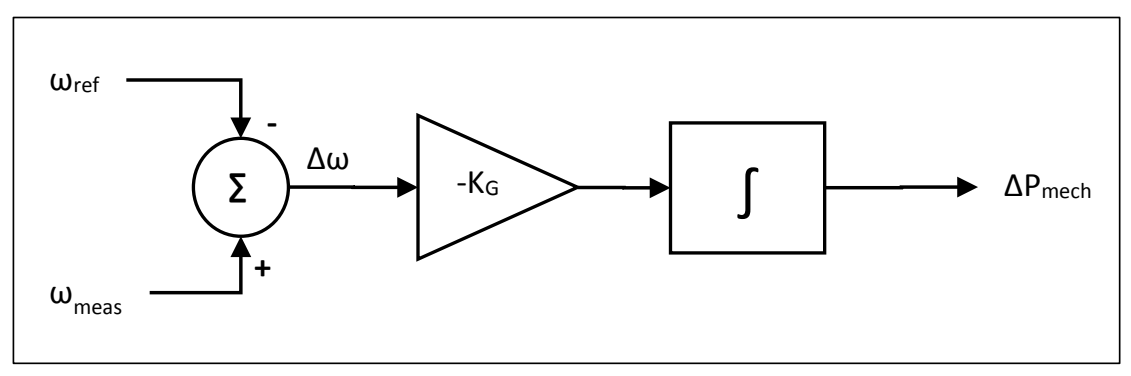

Figure 3.3.1: Isochronous Control

However, when more than one generator exists in a power system, a power sharing scheme must be used in order to prevent the units from "fighting” each other. If more than one unit is kept in isochronous operation, unless their reference frequencies are exactly the same or have a more advanced control system, one unit is going to be a bit above or below the nominal frequency value. This in turn means that this generator will adjust its power output and further deviate from the reference.

For example, if there are two generators in isochronous mode, and unit \#1's reference frequency is set at $60.001 \mathrm{~Hz}$, when that unit detects the system frequency at 60 $\mathrm{Hz}$, it will increase its power output, which will bring the system frequency up. If generator \#2 is set at exactly $60 \mathrm{~Hz}$, in detecting the frequency shift upward, it will decrease its power 
output. This happens continuously until all the power is transferred to unit \#1. To prevent this from happening, droop control is implemented.

In droop control, after a power imbalance, each generator connected to the system will settle at a new operating frequency. For example, considering only one unit, if it is operating at power level $P$ and nominal frequency $f_{0}$, after an increase in load $\Delta P_{L}$ is applied, the generator will increase the power output to $P^{\prime}=P+\Delta P_{L}$ and decrease linearly by $\Delta f$ to settle at frequency $f$ '. This is described by the speed droop characteristic of Fig.

\subsection{2:}

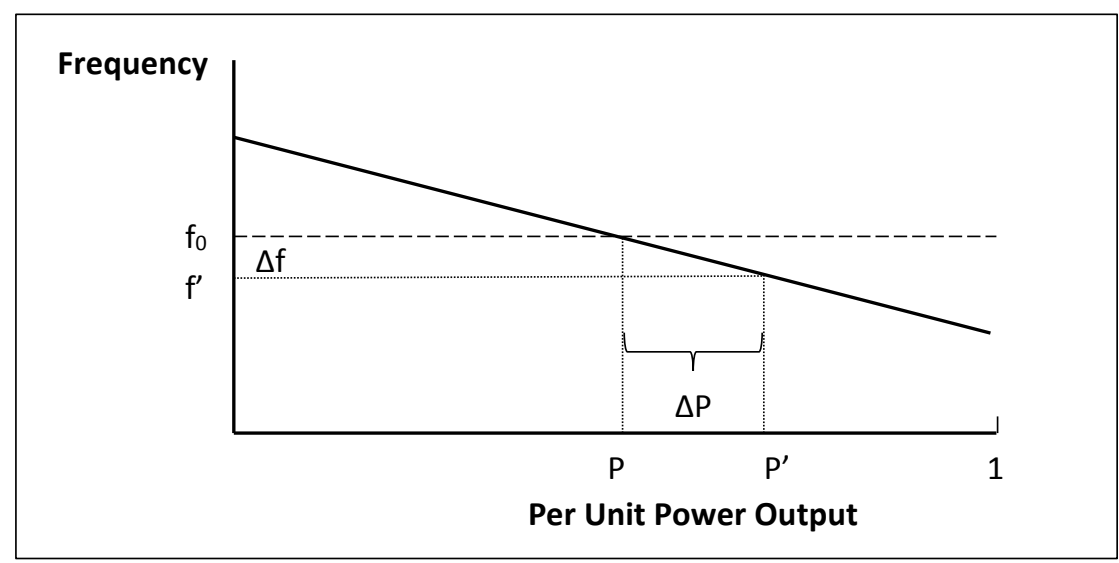

Figure 3.3.2: Speed-Droop Characteristic

In the case of more than one generator, the power imbalance will be shared according to the slope of its droop characteristic. Typically, the slope is determined by the unit's rated power and a system minimum and maximum allowable frequency:

$$
R=-\left(f_{\text {max }}-f_{\text {min }}\right) /\left(P_{\text {max }}-P_{\text {min }}\right)
$$

Thus, in the case of two generators, where one has 50\% more rated capacity, the scenario shown in Fig. 3.3.3 would be observed. As explained in equation 3.3.1, the droop setting of both generators is calculated from the minimum and maximum allowable 
frequency, $f_{\min }$ and $f_{\max }$, respectively, which correspond to $f_{F L}$ and $f_{N L}$, which are the generator's Full and No-Load frequencies, respectively. Since the second generator has a higher rated capacity, its slope is less pronounced.

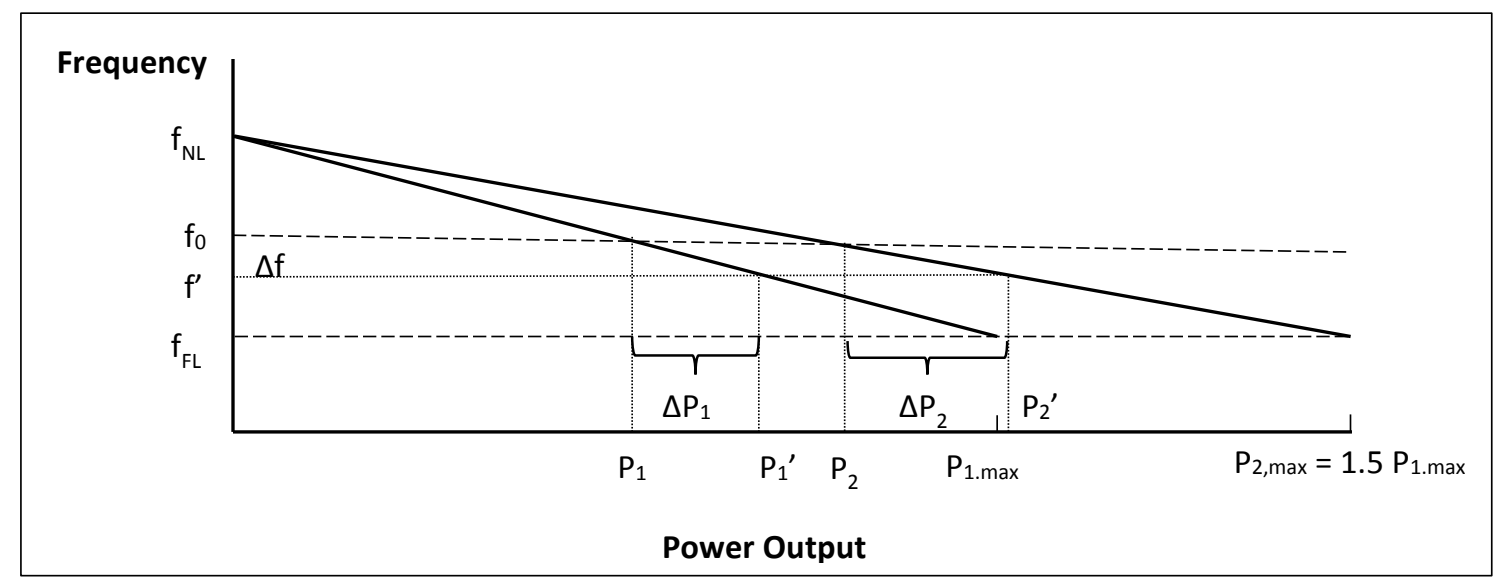

Figure 3.3.3: Speed-Droop Characteristic, Two Generators

Initially, the generators are operating at nominal frequency $f_{0}$; the first unit is delivering $P_{1}$ and the second $P_{2}$. After an increase in load $\Delta P_{L}$, the system frequency is decelerated to $f^{\prime}$, but the units are now operating at $P_{1}{ }^{\prime}=P_{1}+\Delta P_{1}$ and $P_{2}{ }^{\prime}=P_{2}+\Delta P_{2}$, where $\Delta P_{L}=\Delta P_{1}+\Delta P_{2}$. It can be seen how $\Delta P_{2}$ is larger than $\Delta P_{1}$; in fact in this case it is 50\% more. Thus, droop control implements proportional power sharing; in other words, the units increase their output relative to their rated power.

Droop control is implemented by adding a negative feedback with a gain equal to $R$, the droop slope or setting, which brings the frequency error down to zero. As seen in Fig. 3.3.4, the "Load Reference" is the input to be adjusted in order to control the output level of each unit.

Another way to look at the load reference input is the no load frequency, which is essentially the y intercept of the speed-droop characteristic curve (Fig. 3.3.2). By changing 


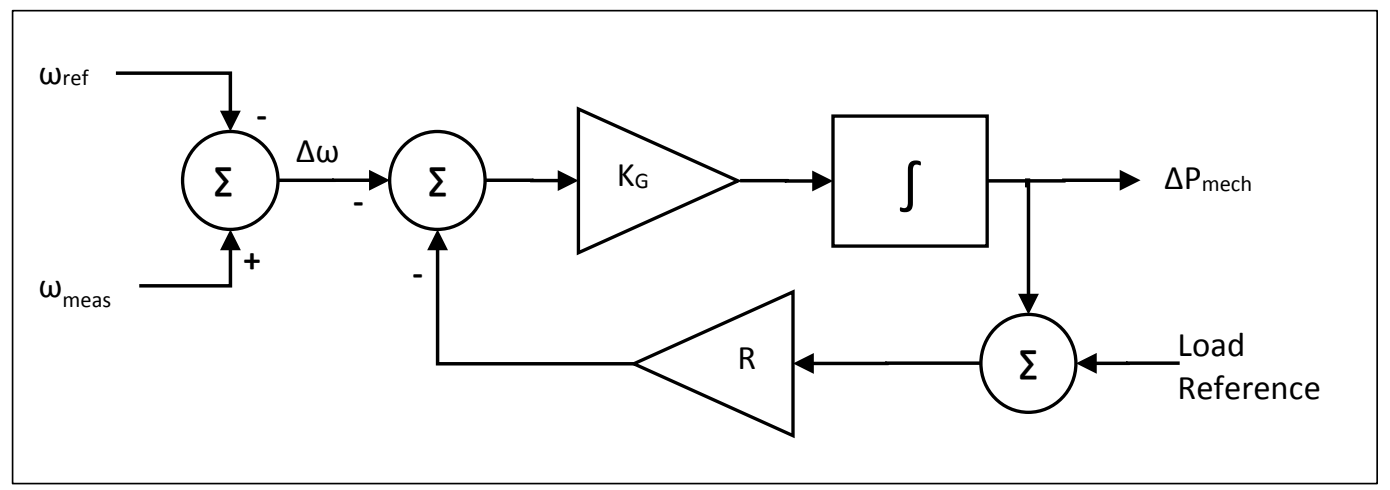

Figure 3.3.4: Droop Control

the load reference or no load frequency upwards or downwards, the power output for any given system frequency can be controlled. For example, suppose there is a $1 \mathrm{MW}$ generator in a $60 \mathrm{~Hz}$ nominal frequency system, with a full-load and no-load frequency of $59 \mathrm{~Hz}$ and $61 \mathrm{~Hz}$, respectively. Thus, the droop slope will be:

$$
R=-(61-59) / 1 e 6=-2 \mathrm{~Hz} / \mathrm{MW}
$$

Mathematically, a unit's output power can be calculated as follows [29]:

$$
P_{i}=\left[\left(P_{i, \text { max }}-P_{i, \text { min }}\right) /\left(f_{F L}-f_{N L}\right)\right]\left(f_{\text {system }}-f_{N L}\right)
$$

If initially the no load frequency is set at $61 \mathrm{~Hz}$, and the system frequency remains at $60 \mathrm{~Hz}$, then the output power would be:

$$
P=[(1 M W-0) /(59-61)](60-61)=500 k W
$$

Or, in other words:

$$
P=-\left(f_{s y s}-f_{N L}\right) / R=-(60-61) /(-2)=0.5 M W
$$

To operate the generator at a higher or lower level, the no-load frequency is simply modified. For example, if $f_{N L}$ is increased to $61.5 \mathrm{~Hz}$, the new power output would be:

$$
P=-(60-61.5) /(-2)=0.75 M W
$$

If the no-load frequency were to be decreased to 60.5: 


$$
P=-(60-60.5) /(-2)=0.25 M W
$$

Conversely, to know at what no-load frequency to set the generator to obtain a given power output:

$$
\begin{aligned}
& f_{N L}=P\left(f_{N L}-f_{F L}\right) /\left(P_{\max }-P_{\text {min }}\right)+f_{s y s} \\
& f_{N L}=-P \cdot R+f_{s y s}
\end{aligned}
$$

For example, to set the $1 \mathrm{MW}$ generator to output $900 \mathrm{~kW}$, the no-load frequency is calculated by:

$$
f_{N L}=0.9(61-59) /(1-0)+60=-0.9(-2)+60=61.8
$$

The previous examples are depicted in Fig. 3.3.5:

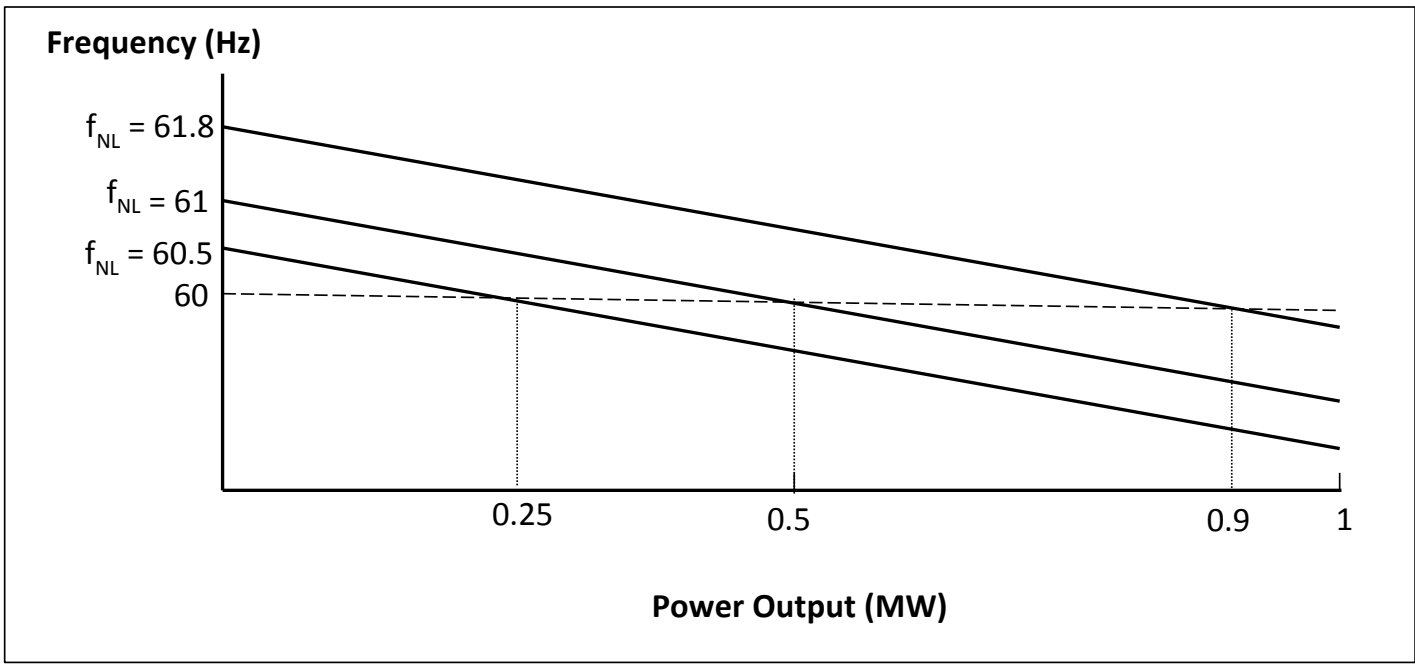

Figure 3.3.5: Adjusting No-Load Frequency

Because droop is a fast-acting primary response control scheme, it is perfect for MGs. As will be seen in Chapter 6, droop control will be implemented to take care of the power sharing and frequency stability in an automatic way in the proposed MG control system. An additional optimization level for secondary and tertiary control will adjust the no-load frequencies, as described above. The Multi-Agent System responsible of this 
higher-level control will be introduced and described in the following chapter. The result will be a stable and efficient MG control scheme. 


\section{CHAPTER 4: MULTI-AGENT SYSTEMS}

Multi-Agent Systems (MAS) hold much promise when the goal is to have a decentralized control scheme. In this chapter, centralized vs decentralized control systems will be outlined and the pros and cons of each will be weighed to see why a distributed structure is desired. The basics of communication networks and dynamic systems will be introduced to give the background for MAS and how they can be applied to Microgrids.

\subsection{Centralized vs Decentralized Control Systems}

Although droop control, as described in Section 3.3, is implemented per generator, when considering Automatic Generation Control (AGC) and Supervisory Control and Data Acquisition (SCADA) for power systems, power control systems have traditionally been implemented in a centralized hub. There are several reasons for this:

1. Computational Power: Historically, wide-area control systems were implemented with hardware-based electronic components, as the availability and accessibility of computing power was limited and therefore software solutions were not sufficient. These systems were highly tailored and very expensive to implement, as they had to be as reliable and stable as possible.

2. Speed: Minding the previous point about computational power, having all information aggregated by one entity means decisions can be made with a global system perspective as fast as they can be processed.

3. Security: Being such an integral part of our modern life, electrical power systems need to be ultra-secure to outside influences. By having all control 
mechanisms in one protected location, the chances of accidental mishaps or intentional sabotage are reduced.

4. Standardization: By having one central entity controlling the whole system, everything is tailored to it and there are no issues of inter-operability or backward compatibility when upgrading or adjusting the system.

However, computational capabilities have exploded in the past decades, making most of the previous reasons obsolete and creating a real push towards control decentralization. To understand this momentum, the downsides of centralized control are outlined next:

1. Single Point of Failure (SPOF): By having one unique entity aggregating all measurements and providing control for the entire network, the system becomes utterly dependent and vulnerable to any problem such entity might face. Backup systems are necessary for each component in such vital infrastructure as the power grid, which adds to the complexity and cost of the system.

2. Scaling: As centralized systems grow in size, their complexity grows at a faster pace. The communication infrastructure has to be readjusted for every addition to the system in an ever-complex mesh. The processing power likewise needs to be upgraded for the added burden; it is said that such systems "do not scale".

3. Customization: The highly tailored nature of centralized systems means that they are inflexible and inherently hard to upgrade; thus they fall behind the latest technological advancements.

4. Cost: All the previous disadvantages have the added drawback of increasing the cost of the control scheme. 
Decentralization is an evolving field of study as computing power becomes faster and more prevalent in all aspects of life. In a decentralized (or distributed) system, the measurement, processing and control actions are divided into clusters, which do not depend on a hierarchical structure and act only on local information. Distributed control systems address all of the previously mentioned downsides of central controllers:

1. Resiliency: Because there isn't one single agent on which all others depend, the system is by design resistant to failures of any given component and able to adapt when fault conditions arise.

2. Scaling: Distributed systems can be designed so that communicational and computational complexity does not increase for each addition to the system. Instead, every additional cluster has the same communication and processing capabilities as all the others, thus the system scales linearly according to the size of the system.

3. Flexibility: By incorporating communications and industrial protocols and standards, decentralized systems can be designed to be adaptable and readymade to be "plug and play". In the case of a MG, one can envision a system where if a new load or Distributed Generator (DG) is added, it can immediately start communicating with the adjacent clusters and the system as a whole can automatically reach a stable operating condition.

4. Cost: Because distributed computation has become so inexpensive, and software control and communication systems have become so sophisticated, implementing such a decentralized control system is now not only economically 
feasible, but more cost effective than centralized solutions, especially considering the ability to easily and cheaply expand the system.

Decentralized control systems do present some challenges and concerns, one of them being system security and stability. As was previously mentioned, such a system can be designed to be resistant to a loss of any given cluster and maintain stability; this will be further described in Chapter 5. Regarding cyber-security, the latest security measures can be implemented to keep attackers at bay. Furthermore, the system does not depend on the internet and so the communications can be as secure as any centralized system.

The main drawback of a distributed system is the trade-off in speed when solving a control problem. A centralized system has a system-wide perspective of the current conditions and thus can solve an optimization problem as quickly as its processing power allows it to. When considering Economic Dispatch (ED) for a MG, the computational requirements are not high at all, therefore a central controller can solve it immediately. As will be seen in Chapter 5, distributed optimization depends on several communication iterations to reach an agreement on the optimal solution.

In the proposed MG scheme, this disadvantage is mitigated by having droop as the primary control system because it acts locally and immediately. Decentralized communication using diffusion (Ch. 5) acts as secondary control to restore the frequency of the MG to the nominal. Additionally, diffusion jointly performs distributed optimization, which acts as the tertiary control or ED of the MG; this is all demonstrated in Chapter 6. 


\subsection{NETWORKS AND DYNAMIC SYSTEMS}

Before considering a network of agents, some concepts and nomenclature need to be established. A graph is the term used for the mathematical representation of a physical (or virtual) network of nodes or vertices interconnected by edges. A graph $G$ is described by the pair $(V, E)$ where $V=\{1, \ldots, n\}$ represents the finite, nonempty set of nodes, and $E \subseteq$ $V_{x} V$ represents the ordered set of edges, or connections, from one node to the other. An edge $(i, j) \in E$ describes a communication link from node $i$ to node $j$. In a directed graph, $(i, j) \neq(j, i)$, meaning that the edges represent one-way communication. However, in an undirected graph, $(i, j)=(j, i)$, so the communication is bidirectional. Self-edges are denoted by $(i, i)$; although at first it may seem counterintuitive, in Chapter 5 , the significance of selfcommunication will be described. [30] [31]

Graphs are considered strongly connected when there exists a directed path to and from any one node to the other, and simply connected when such path is undirected. The set of neighbors for node $i, N_{i}$, represents all the nodes which share an edge with $i$, and the degree of node $i$ is given by $\left|N_{i}\right|$. The adjacency matrix $A=\left[a_{i j}\right] \in \mathbb{R}^{n x n}$ represents the nonnegative weights that any node $i$ gives to communications received from node $j$, granted $j \in N_{i}$, otherwise $a_{i j}=0$; this can also be thought of as a trust factor. These concepts are shown in Fig. 4.2.1.

It can be seen how the edges of the network in Fig. 4.2.1 are bidirectional. Looking specifically at node 1 , the neighborhood $N_{1}$ is depicted by the light grey area with nodes 2 , 3 and 4 as direct neighbors. Nodes 5 and 6 are also part of the graph $G$, but have to communicate with node 1 through other nodes. Also, node 1 is the only one with a self- 


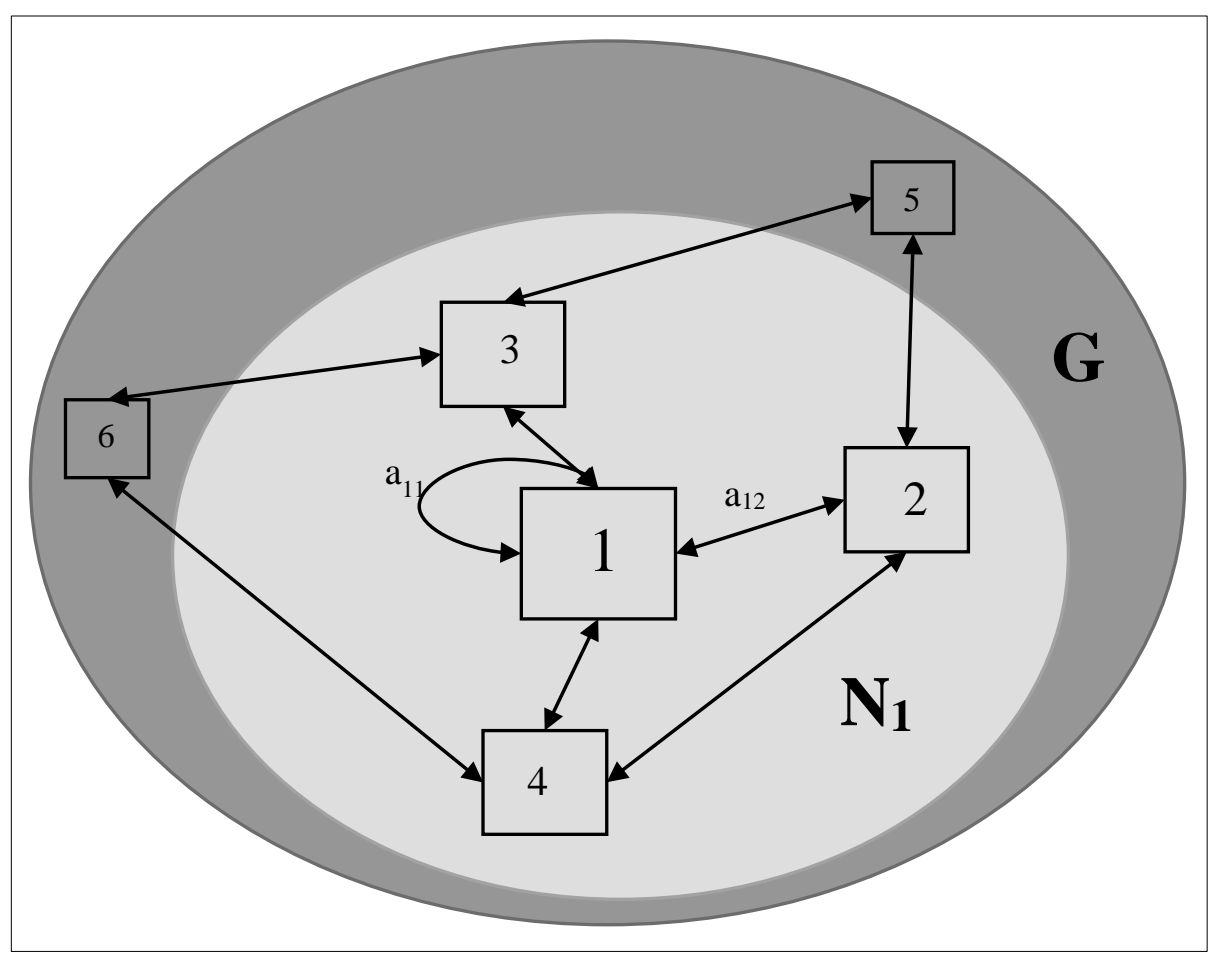

Figure 4.2.1: Graph Example

edge. The edge matrix of this network can be easily described (assuming all weights are of value 1):

$$
E=\left\{\begin{array}{llllll}
1 & 1 & 1 & 1 & 0 & 0 \\
1 & 0 & 0 & 1 & 1 & 0 \\
1 & 0 & 0 & 0 & 1 & 1 \\
1 & 1 & 0 & 0 & 0 & 1 \\
0 & 1 & 1 & 0 & 0 & 0 \\
0 & 0 & 1 & 1 & 0 & 0
\end{array}\right\}
$$

Because the edges are bidirectional, it can be seen that $E=E^{T}$, meaning $E$ is a symmetric and balanced matrix. The Laplacian matrix $L=\left[l_{i j}\right] \in \mathbb{R}^{n \times n}$ is defined as:

$$
l_{i j}=\left\{\begin{array}{cc}
-1, & j \in N_{i} \\
\left|N_{i}\right|, & j=i \\
0, & \text { otherwise }
\end{array}\right.
$$

In the case of the network in Fig. 4.2.1, the Laplacian would be: 


$$
L=\left\{\begin{array}{rrrrrr}
3 & -1 & -1 & -1 & 0 & 0 \\
-1 & 3 & 0 & -1 & -1 & 0 \\
-1 & 0 & 3 & 0 & -1 & -1 \\
-1 & -1 & 0 & 3 & 0 & -1 \\
0 & -1 & -1 & 0 & 2 & 0 \\
0 & 0 & -1 & -1 & 0 & 2
\end{array}\right\}
$$

In this example, and for all balanced and symmetric adjacency matrices, the Laplacian is also balanced and symmetric. The Laplacian matrix is useful in analyzing such things as network stability and performance, mainly through the eigenvalues of $L$ :

$$
e_{L}=\left\{\begin{array}{r}
0 \\
1.59 \\
2 \\
3 \\
4.41 \\
5
\end{array}\right\}
$$

As explained in [31], $L$ has a simple 0 eigenvalue (meaning there is only one) if and only if the network is connected for an undirected graph, which can be clearly seen in Fig. 4.2.1. The second eigenvalue, 1.59, describes the algebraic connectivity of the network, which quantifies the rate of convergence of the consensus algorithm, as described in Chapter 5.

In the case of time-variant networks, where the edges might change or nodes might come in and out of the graph, stability and convergence is guaranteed as long as the graph remains connected and the weights are updated accordingly. In the case of Microgrids, new nodes or agents might come in and out of the network as more generation assets or loads are added to the system. Communication channels might also be blocked or reintroduced, 
and so the goal is for a stable and adaptive network. It will be seen in Chapter 5 how the weights are chosen for optimum performance and robustness.

\subsection{Multi-Agent Systems}

The abstract concept of Multi-Agent Systems (MAS) has a wide variety of applications and definitions. As loosely defined in [32], "Multiagent systems are those systems that include multiple autonomous entities with either diverging information or diverging interests, or both.” Being traditionally software or hardware entities, MAS can be programmed to behave and interact with others in any manner conceivable. Hence, MAS have immense flexibility and can be tailored to a myriad of purposes. There are examples of competing agents, as in the case of financial trading agents, or battling robots, just to name a couple. However, in this thesis, cooperative agents are mainly considered, as they are all programmed to collaborate towards a common goal.

For the end objective of a decentralized Microgrid (MG) control scheme, autonomous agents can be employed at each node of the electrical system; next the MAS agents to be used throughout the thesis will be defined:

- Load Agents (LA) are associated to load feeders and are of a stochastic nature, as the behavior of consumers can't be precisely predicted. They can measure and broadcast the current power requirements of their connected loads.

- Renewable Agents (RA) are those associated with Renewable Energy Sources (RES) and are also stochastic, as wind and solar conditions similarly can’t be exactly forecasted. RES are considered negative loads for the purposes of the control scheme being developed, as they operate at their Maximum Power Point 
Tracking (MPPT) output and can’t be dispatched on command. They measure and communicate their current power output.

- Distributed Generator Agents (DGA) are associated with each DG and they measure and control their current output power and the cost function only known to them. As described in Chapter 5, they calculate their marginal cost in order to perform distributed optimization.

- Energy Storage Agents (ESA) behave in the same manner as DGAs, except they can also store energy and thus can have a negative power output. They have an associated cost function only known to them and also use their current marginal cost in optimization; this cost function is explained shortly.

- Grid Agents (GA) are associated to the MG's Point of Common Coupling (PCC) and they monitor the power in or out of the MG, as well as the current electricity rate being charged by the utility. They use this rate, which can also be thought of as a constant marginal cost in optimization:

$$
\begin{gathered}
F_{G}=r_{u} \cdot P_{G} \\
\frac{d F_{G}}{d P_{G}}=r_{u}
\end{gathered}
$$

Where $F_{G}$ is the cost of the power $P_{G}$ being drawn from, or fed to, the grid; $r_{u}$ being the current electric rate being charged or offered by the utility grid. Thus, the cost is a linear function of the power and the marginal cost $d F_{G} / d P_{G}$ is a scalar value equal to the electric rate. As described in Section 3.1, each agent's marginal cost will be equal to each other's when operating in the most cost-effective mode (Lagrange multiplier). Hence, because the marginal cost at any given time is independent of the power being drawn from the utility 
in a grid-connected MG with the described rate scheme, all DGs will converge to the point where their marginal cost is equal to the electric rate.

Considering the cost function for the ESA with this in mind, the Energy Storage System (ESS) should ideally charge when the rate of electricity (or marginal cost) is low and discharge when high. There seems to be a lack of agreement on what such a cost function should look like in the literature. In [33], the authors present a function which takes into consideration the rate of charge or discharge, the number of cycles and the depth of discharge; these factors are specific to Battery systems (BESS). A more general cost function is proposed, which is technology-agnostic:

$$
\begin{gathered}
F_{B}=a+b\left(P_{B}+3 P_{B, \text { max }}(1-S O C)\right)+c\left(P_{B}+3 P_{B, \text { max }}(1-S O C)\right)^{2} \\
\frac{d F_{B}}{d P_{B}}=b+c\left(2 P_{B}-6 P_{B, \text { max }}(S O C-1)\right) \\
-P_{B, \text { max }} \leq P_{B} \leq P_{B, \text { max }}
\end{gathered}
$$

Where $F_{B}$ is the cost of the power $P_{B}$ being charged or discharged from the ESS, $P_{B, \max }$ is the maximum charge or discharge rate, SOC is the current State of Charge of the ESS and $a, b$ and $c$ are the quadratic coefficients similar to those in Eq. 3.1.4. $d F_{B} / d P_{B}$ is the derivative of the cost function with respect to the power. As can be observed, the marginal cost is proportional to the power drawn and inversely proportional to the state of charge, meaning the marginal cost will be lower as the state of charge approaches $100 \%$. Fig. 4.3.1 shows how cost function 4.3.3 looks at different SOC levels.

Starting from $S O C=0 \%$ where only negative (or charging) power can be drawn, it can be seen that the cost is quite high, which provides an incentive to charge. As SOC 


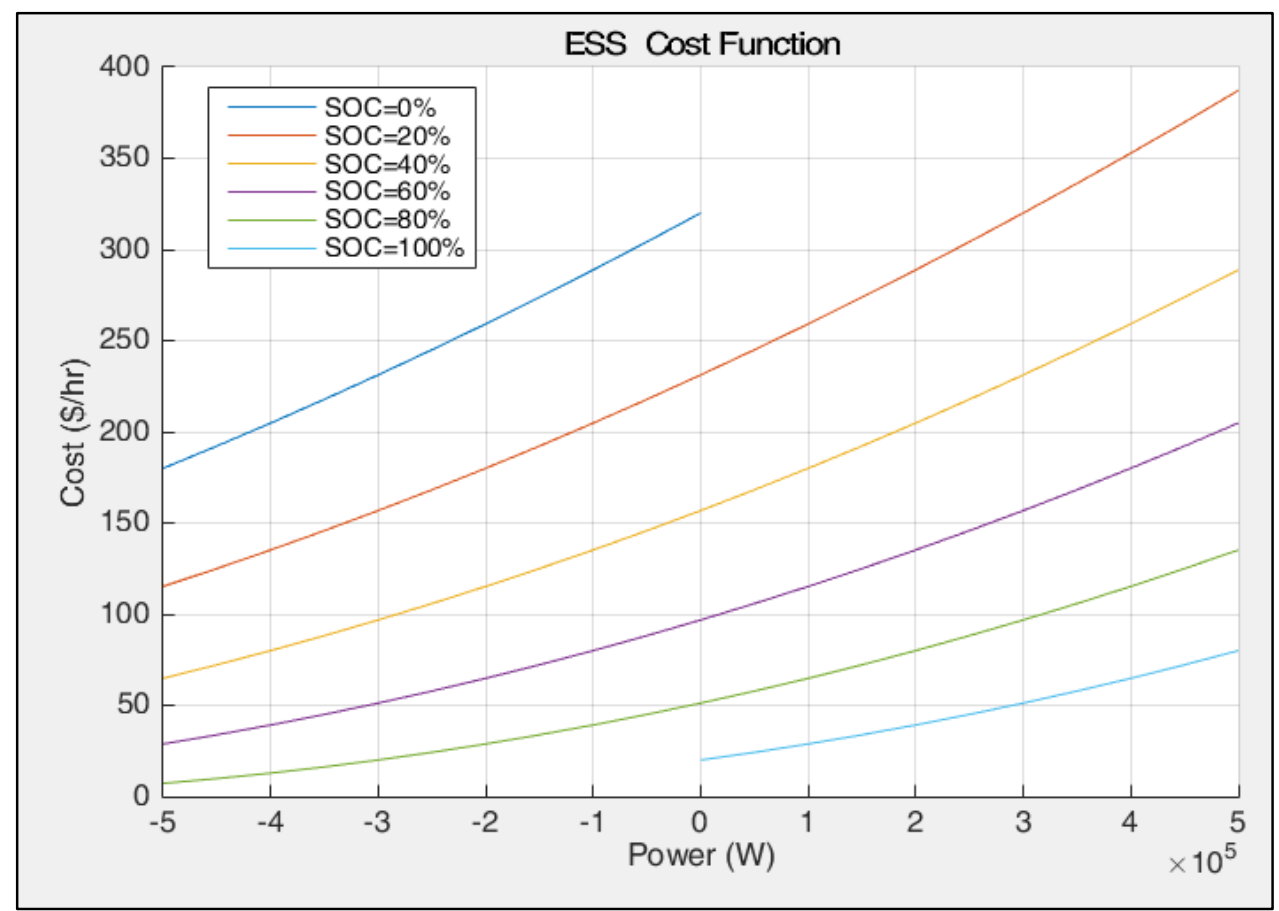

Figure 4.3.1: Energy Storage System Cost Function

increases, the curve effectively shifts to the right and the costs decrease for any given power level until 100\%, where only positive (or discharging) power can be provided.

Looking at the curves for the marginal cost in Fig. 4.3.2, it can be seen that as the SOC decreases, the marginal cost increases. When the SOC is critically low, the marginal cost is so high the Lagrangian point will undoubtedly shift the ESS power point towards charging (negative) power. Likewise, when the SOC is quite high, the marginal cost curve is shifted downwards and more power can be discharged from the ESS. Since the ESS cost function depends on both output power and SOC, the curves can be visualized in three dimensions. As shown in Fig. 4.3.3, as the power level increases, the cost increases, and as the SOC increases, the cost decreases. 


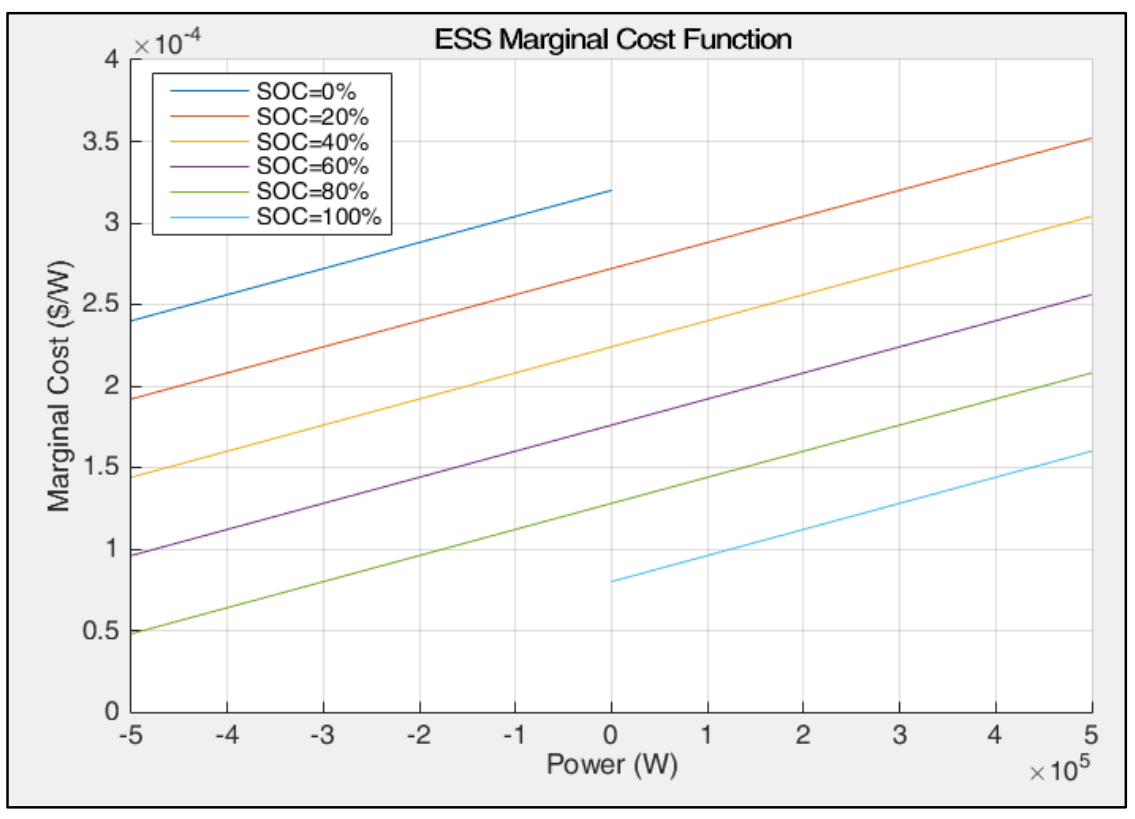

Figure 4.3.2: Energy Storage System Marginal Cost Function

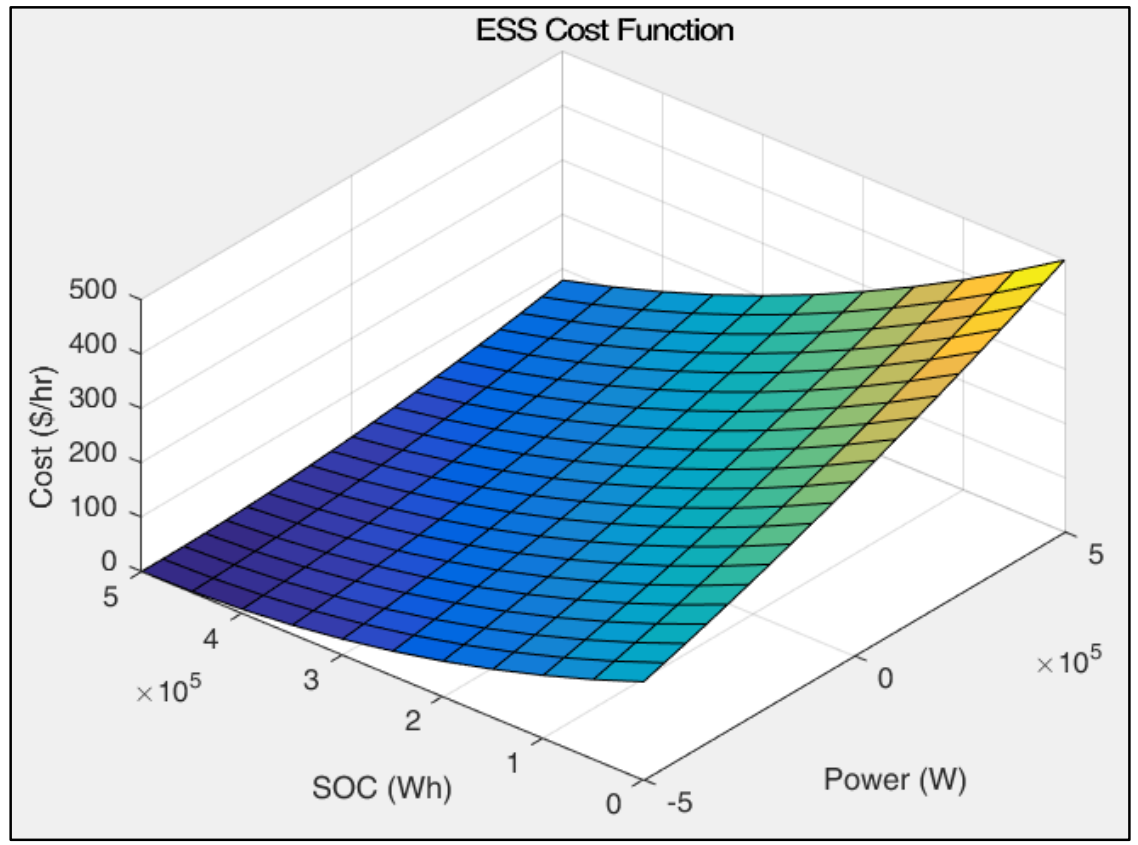

Figure 4.3.3: Energy Storage System Cost Function Surface 


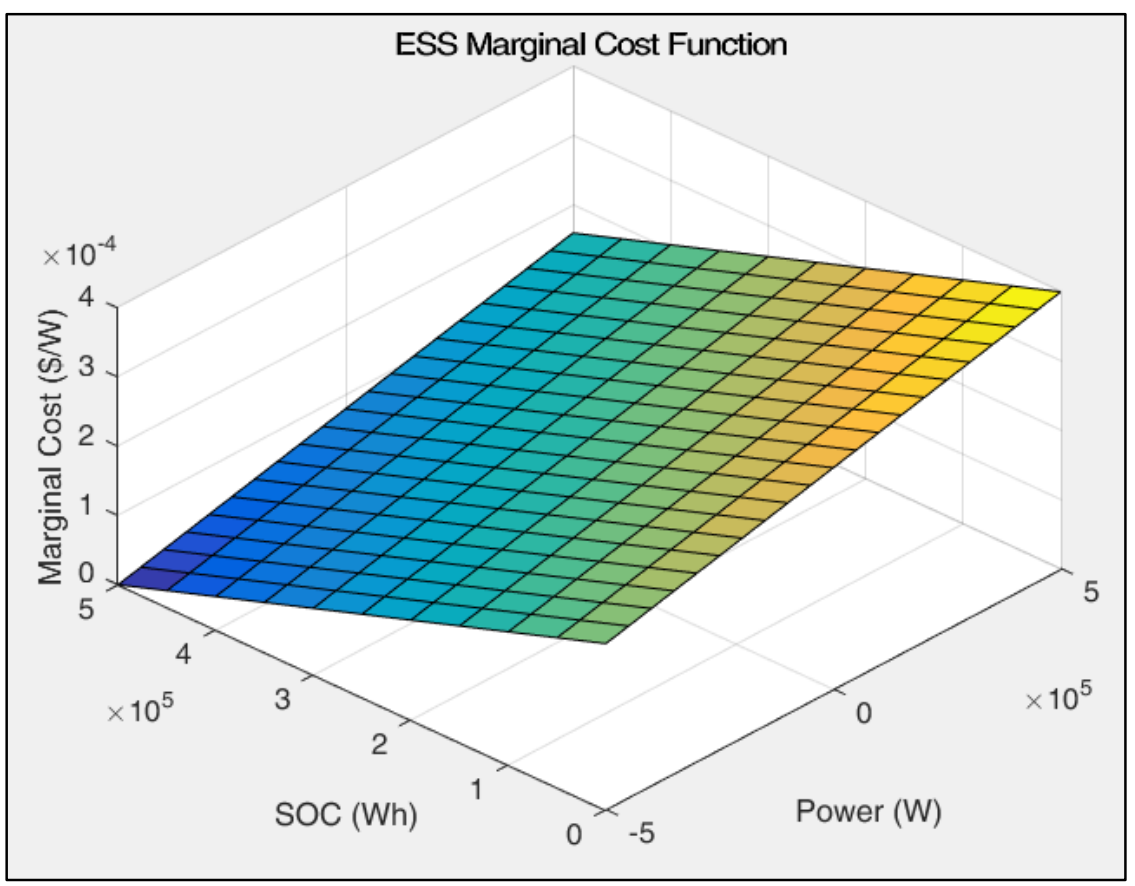

Figure 4.3.4: Energy Storage System Marginal Cost Function Surface

Similarly for the marginal cost, as the power output increases, the marginal cost increases; however this can be thought of in another way. As mentioned previously, the objective is to have an ESS, which charges during low marginal cost times and discharges during high cost times. Additionally, the SOC provides another input to determine what the ESS should do according to how much energy it has available. In Chapter 6, it shall be seen how this translates in an actual system.

Shifting the attention back to the MAS being described, each entity can only communicate with its adjacent neighbors (which are defined as the neighborhood of the node, as described in the previous section), and each entity has information only known to it (diverging information). Additionally, as seen above, the GA, DGA and ESA nodes have cost functions associated to their power input and output, which are specific to each asset. Each agent seeks to minimize its own cost (diverging interests), but as described in Section 
3.1, the global objective is to minimize the total cost. Thus, as will be described in Chapter 5, the decentralized optimization will adjust each agent's power level to reach this goal.

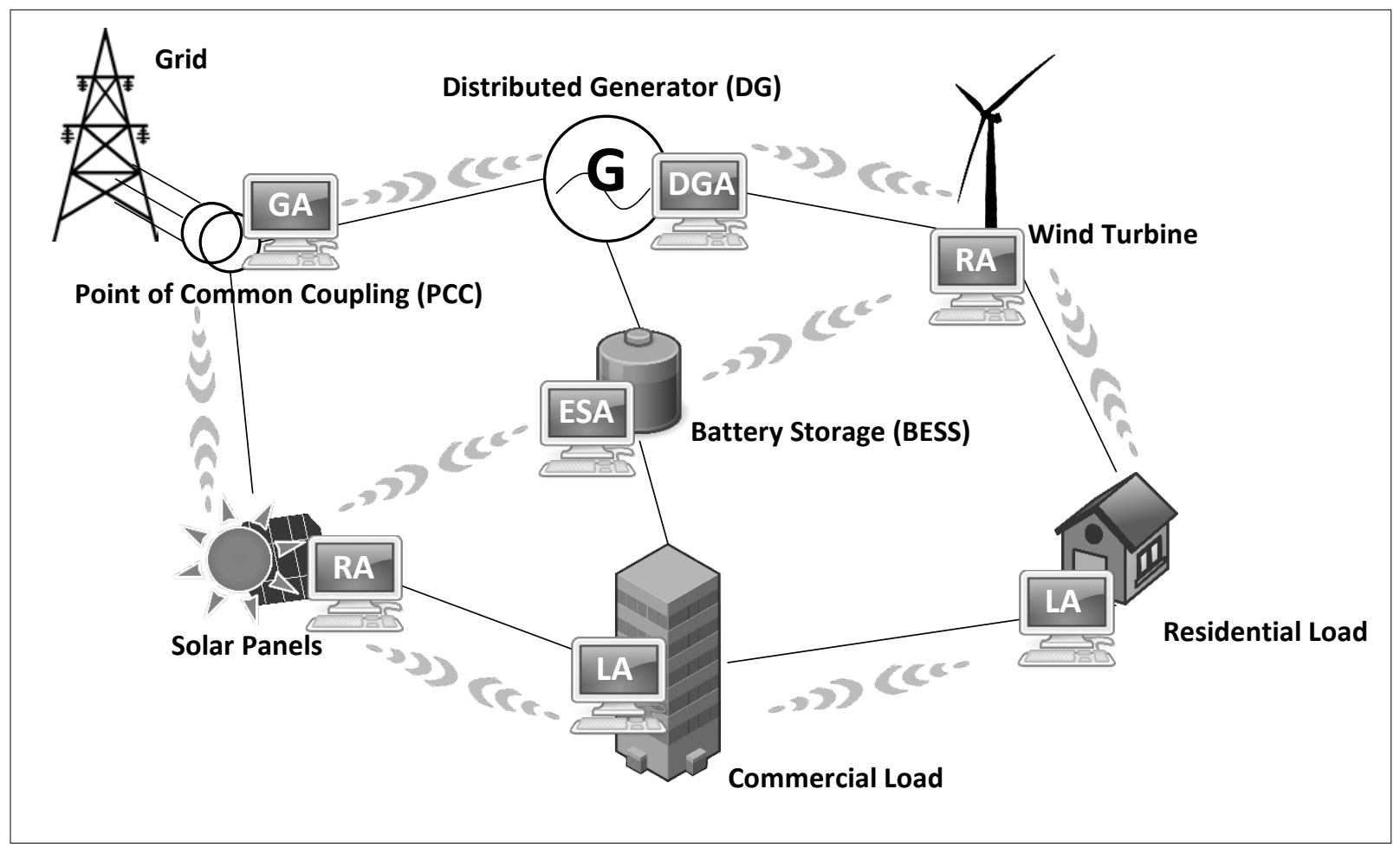

Figure 4.3.5: Microgrid MAS Agent Assignments

Considering the MG diagram from Chapter 2, in Fig. 4.3.5 each node of the system is assigned its respective agent. Communication between agents can be done through wired or wireless infrastructure; the latter being the more attractive option when system flexibility and "plug and play" capabilities are desired.

Luckily, software standards have been created for the interoperability of intelligent agents running on different platforms. The Foundation for Intelligent Physical Agents (FIPA) specifies how agents should behave and how they should communicate with each other using Agent Communication Language (ACL). In this implementation of a MG decentralized control system, the Java Agent DEvelopment Framework (JADE) was used 
to program the previously mentioned agents. JADE employs FIPA and ACL standards and provides a flexible foundation on which to encode whatever behavior is necessary for the system being developed. This will be further described in Chapter 6 . 


\section{CHAPTER 5: DIFFUSION COMMUNICATION}

When considering decentralized communication for Microgrids (MG), there have been several proposals in the literature to apply the consensus strategy, as will be described below [21] [17] [18] [19] [16]. As explained in [5], the diffusion strategy is not only faster but also more robust when it comes to disseminating information across a network. Furthermore, diffusion allows for distributed optimization to achieve a global objective, which will be applied to Economic Dispatch (ED) of a MG. Only [20] has so far described utilizing distributed optimization for ED of MG resources using consensus; however, primary control was not outlined. The strategy proposed is faster, more robust, and with the added stability of droop control and a more sophisticated Energy Storage System (ESS) cost function, it translates into a more comprehensive control scheme.

\subsection{INFORMATION SHARING IN NETWORKS}

Keeping the nomenclature for networks presented in Section 4.2, the main strategy for decentralized information propagation through a network is called consensus. In consensus, each node combines the current states of its neighboring nodes according to the weights assigned by the adjacency matrix A:

$$
x_{i}[k]=\sum_{j=1}^{n} a_{i j}[k-1] \cdot x_{j}[k-1], \quad \mathrm{i}=1, \ldots, \mathrm{n}
$$

Where $x_{i}[k]$ denotes the $i^{\text {th }}$ node's state at iteration $k$, and $a_{i j}$ is the weight node $i$ assigns to information from node $j$, as defined in the adjacency matrix. Note that the weights also depend on the iteration $k$, meaning the network topology can change with time, 
as nodes come in and out of the picture or communication paths are blocked or reintroduced. Eq. 5.1.1 can also be described in matrix form:

$$
x[k]=A[k-1] x[k-1]
$$

Convergence (or consensus) is achieved when $x_{i}[k] \rightarrow x_{j}[k]$ as $k \rightarrow \infty$ for all $i, j=1, \ldots, n$; this is guaranteed in a connected network where $A$ is row-stochastic [31]. Recall from Section 4.2 that a connected network is one where there exists a path from any one node to another in an undirected graph (bidirectional communication); for simplicity, only undirected and connected networks are considered henceforth. A row-stochastic matrix is one where the elements of each row sum up to 1 , meaning the combined weights of the information coming from all neighbors (including self-communication) add up to one. This is also called a left-stochastic matrix, as $A$ satisfies $A^{T} \mathbb{1}=\mathbb{1}$, where $\mathbb{1}$ is an $n \times 1$ vector of all ones.

When consensus converges, the equilibrium reached is actually the average of the initial values of all the nodes participating. Therefore, if the number of total nodes $n$ is known, the total sum can also be determined. In the case of a MG, this can be used to broadcast the total power requirement of the system, which can then be used for ED of the Distributed Energy Resources (DERs), as will be explained shortly.

As mentioned in Section 4.2, the algebraic connectivity of a network, which is given by the second eigenvector of the Laplacian of the $A$ matrix, affects the speed of convergence. Thus, assigning the weights for the adjacency matrix is nontrivial, as the stability and performance of the system depends on it. The Metropolis Rule is widely used, as it is doubly-stochastic (both row and column, or left and right, stochastic) and has been 
shown to guarantee stability, adaptation to topology changes, and near-optimal performance [5]:

$$
a_{i j}= \begin{cases}\frac{1}{\max \left(n_{i}, n_{j}\right)}, & i \in N_{j} \backslash\{j\} \\ 1-\sum_{i \in N_{j} \backslash\{j\}} a_{i j}, & i=j\end{cases}
$$

In other words, the weights from $i$ to $j$ will depend on the maximum number of neighbors either node has, and the self-weight will bring the total of each row (and column) in $A$ to 1 . By recalculating the weights after the network changes in any way, the Metropolis rule provides the ability to adapt to changing topologies. Referring back to Fig. 4.2.1, and taking the topology matrix defined by Eq. 4.2.1, the adjacency matrix with the Metropolis rule would be:

$$
A=\left\{\begin{array}{cccccc}
1 / 4 & 1 / 4 & 1 / 4 & 1 / 4 & 0 & 0 \\
1 / 4 & 1 / 12 & 0 & 1 / 3 & 1 / 3 & 0 \\
1 / 4 & 0 & 1 / 12 & 0 & 1 / 3 & 1 / 3 \\
1 / 4 & 1 / 3 & 0 & 1 / 12 & 0 & 1 / 3 \\
0 & 1 / 3 & 1 / 3 & 0 & 1 / 3 & 0 \\
0 & 0 & 1 / 3 & 1 / 3 & 0 & 1 / 3
\end{array}\right\}
$$

As can be seen, all rows and columns add up to 1, which, as previously mentioned, is one of the conditions for convergence (specifically the rows). Following with this $A$ matrix, the speed of convergence can be simulated and then compared with the proposed diffusion method, which will be explained in the next section.

To examine a potential real-world scenario and referring to Fig. 4.2.1, suppose node 1 was an Energy Storage System (ESS), node 2 was a commercial load, node 3 was a DG, 
node 4 was a solar array, node 5 was a wind turbine and node 6 was the Point of Common Coupling (PCC) to the grid:

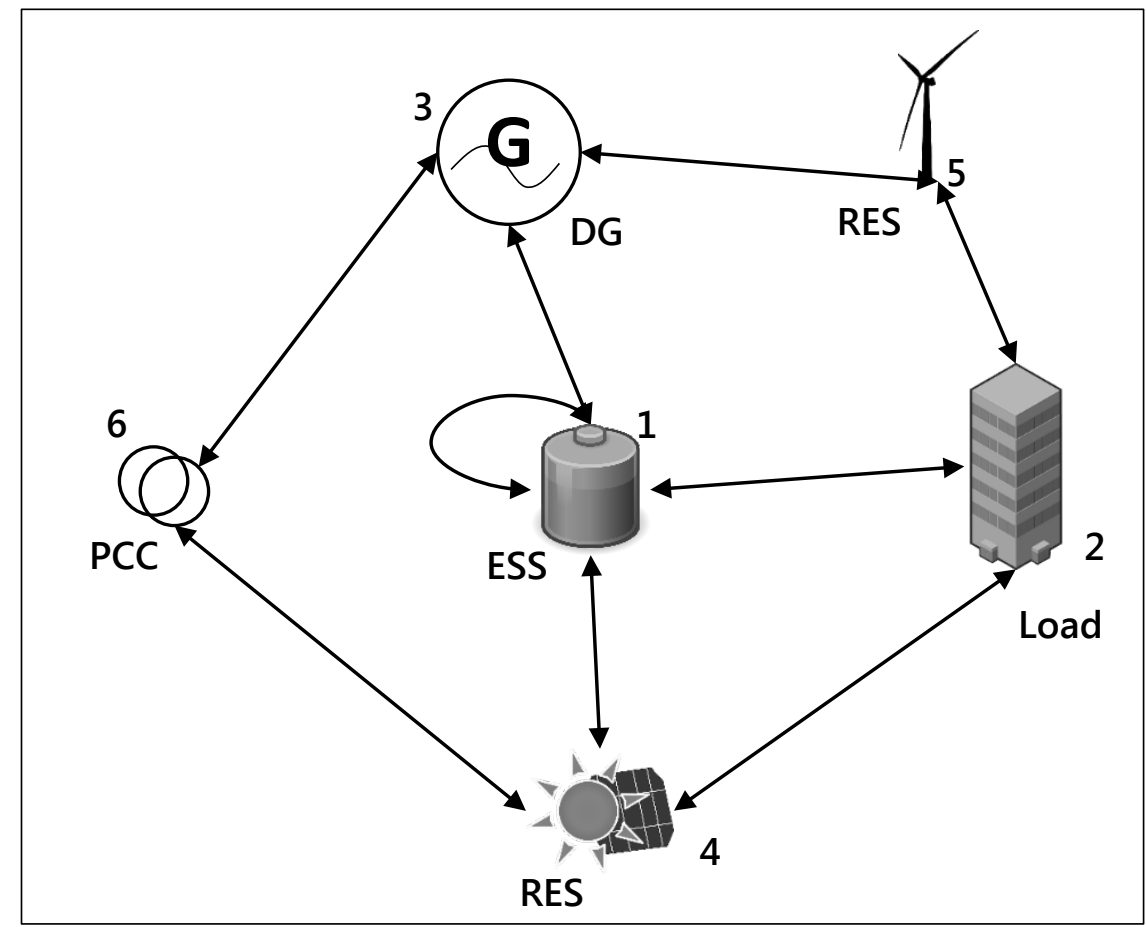

Figure 5.1.1: MG Network Node Layout

The aim is to determine how much power has to be provided by the DG, ESS and grid; the power being drawn by the load will be considered positive, and the power injected by the Renewable Energy Sources (RES) will be considered negative. Consensus will be used where the states of the generating assets are initially set to zero.

Suppose the load being drawn was $100 \mathrm{~kW}$, the solar array was providing $10 \mathrm{~kW}$, and the wind turbine was providing $15 \mathrm{~kW}$. The initial states would be:

$$
x[0]=[0,100 e 3,0,-10 e 3,-15 e 3,0]
$$


By running the consensus algorithm until a desired level of precision, the process of convergence can be visualized. In this case, a precision of five significant figures was used, and convergence was achieved in 14 iterations:

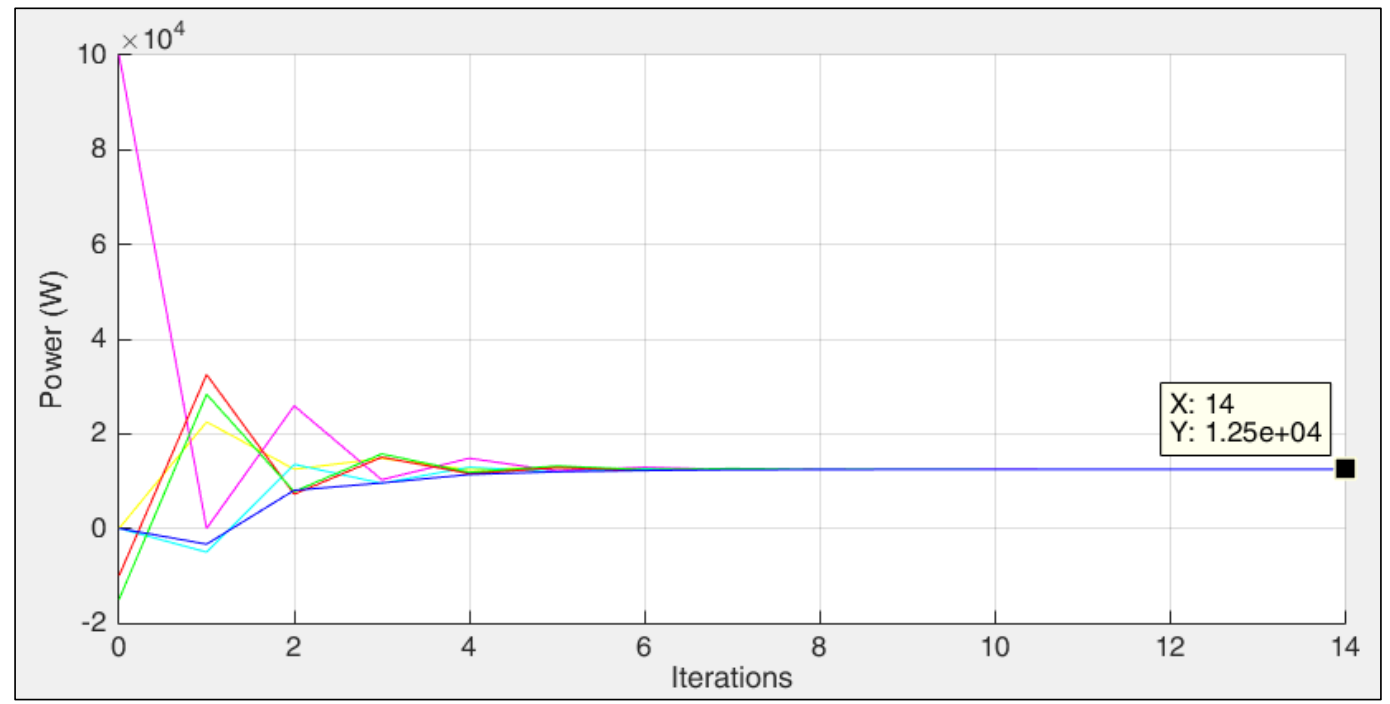

Figure 5.1.2: Consensus Convergence

It can be seen how each node begins at its initial state, magenta being the load at node two, red being the solar panel at node four, green being the wind turbine at node five, and the other nodes starting at zero. All the nodes converge at $12.5 \mathrm{~kW}$; which is the average of the values of $x[0]$. Also, this value multiplied by the number of nodes will give the net power needed by the MG: $12.5 e 3 * 6=75 e 3$, which is equal to the load minus the RES output (100 kW-10 kW-15 kW = $75 \mathrm{~kW})$. In Section 5.4, it will be explained how this information can then be used to perform decentralized ED. Now, diffusion will be described and compared to consensus. 


\subsection{Diffusion STRATEgIES}

Inspired by adaptation and learning in nature, such as with schools of fish or swarms of bees, diffusion builds upon consensus to create a faster and more adaptive strategy. In [34], the authors describe how a stochastic gradient is included in an adaptation phase of an agent's process in order to more quickly diffuse information through the network; this is termed Combine Then Adapt (CTA):

$$
\text { CTADiffusion : }\left\{\begin{array}{c}
\phi_{i, k-1}=\sum_{j \in N_{i}} a_{i j} x_{j, k-1} \\
x_{i, k}=\phi_{i, k-1}-\mu_{i} \hat{s}_{i, k}\left(\phi_{i, k-1}\right)
\end{array}\right.
$$

Where $x_{i, k} \in \mathbb{R}$ denotes the state of agent $i$ at time $k, \phi_{i, k}$ is the intermediate variable for agent $i$ at time $k, \mu_{i}$ is a nonnegative updating parameter of agent $i$, and $\hat{s}_{i, k}(\phi)$ is the stochastic gradient for agent $i$ of the intermediate state $\phi$ at time $k$. The stochastic gradient is simply calculated on the difference of $\phi$ from one iteration to the other; thus, each agents keeps track of its gradient and updates the combination of all its neighbors according to it. There is also Adapt Then Combine (ATC) diffusion, which inverts the steps in Eq. 5.2.1, but only CTA is used hereafter.

Applying this algorithm to the same example described in the previous section and illustrated by Fig. 5.1.1 and initial conditions shown in Eq. 5.1.5, the following result is obtained (same Metropolis weights are also used): 


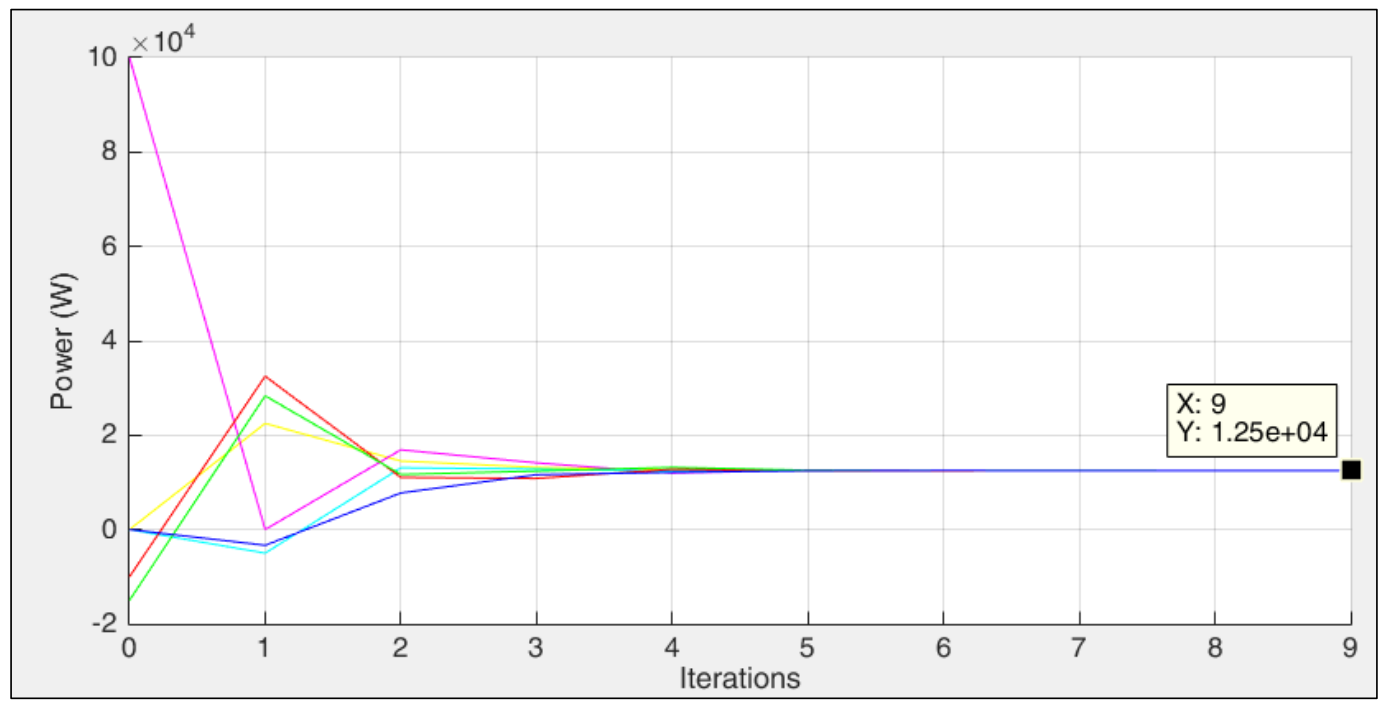

Figure 5.2.1: Diffusion Convergence

The same level of precision (five significant figures) is achieved in considerably less iterations by diffusion (9 vs 14; 36\% less). When compared to Fig. 5.1.2, diffusion seems to "damp” the overshoot shown, especially by the load agent (node two in magenta).

Of course the step size $\mu$ affects the performance and stability of the diffusion algorithm and must be tailored to each application and network; in this example it was found by trial and error that the optimum $\mu$ was 0.09 . As shown in [5], diffusion guarantees stability with sufficiently small step sizes; additionally, diminishing step sizes can ensure the convergence of the solution to the definite value. However, this removes the ability to track a drifting solution; therefore there is a tradeoff between precision and adaptability.

In the case of a MG control system, diffusion is preferred over consensus because for a predetermined stringency in precision, the performance is significantly better. Thus, the step size can remain constant after being adjusted to the desired precision level, and the network will continuously outperform consensus. In the next section, this adaptability concept will take on a whole different meaning. 


\subsection{DECENTRALIZED OPTIMIZATION}

As mentioned in Chapter 3, the marginal cost of a DG is the gradient of its cost function; this is where diffusion and Economic Dispatch (ED) come together. The previous section described how diffusion includes a stochastic gradient in the information sharing (also known as estimation) problem. For decentralized optimization of MG resources, the marginal cost is used instead. First, the global problem is defined [6]:

$$
\begin{gathered}
J_{g l o b}(w)=\sum_{i=1}^{n} J_{i}(w) \\
\min _{w} J_{g l o b}(w)
\end{gathered}
$$

Where $J_{i}$ is the cost function of agent $i$ defined over the real-valued vector of arguments $w \in \mathbb{R}^{n}$; therefore the objective is to minimize the global cost $J_{g l o b}$, which is the sum of all the individual cost functions. The cost functions need not be the same for each agent, and they need not be minimized at the same $w$ vector [7]; this shall be the case for the MG where each agent has its own cost function. According to [5], all $J_{i}$ need to be convex and differentiable; a convex function is one for which a line segment between any two points on its graph lies on or above it. Furthermore, at least one $J$ needs to be strongly convex in order for $J_{\text {glob }}$ to also be strongly convex, meaning there is only one global minimum solution. Strong convexity is the same as convexity except the line segments must be strictly above the graph. Mathematically, convexity can be defined through the second derivative of $J$ :

$$
J(w) \text { is convex } \Leftrightarrow \nabla_{w}^{2} J(w) \geq 0
$$




$$
J(w) \text { is strongly convex } \Leftrightarrow \nabla_{w}^{2} J(w)>0
$$

In the case of a MG, the cost functions of the distributed resources are all strongly convex with the exception of the Grid Agent (GA), which is only convex (linear functions are both convex and concave, satisfying Eq. 5.3.3). The Distributed Generator Agent's (DGA) and Energy Storage Agent's (ESA) cost functions are strongly convex since they are positive quadratic functions. Thus, $J_{\text {glob }}$ is also strongly convex and can reach a global minimum through optimization.

Similar to CTA diffusion for the estimation problem (Eq. 5.2.1), CTA can be used for distributed optimization by adopting the gradient of the cost function instead of the stochastic gradient:

$$
\text { CTAOptimization: }\left\{\begin{array}{c}
\phi_{i, k-1}=\sum_{j \in N_{i}} a_{i j} w_{j, k-1} \\
w_{i, k}=\phi_{i, k-1}-\mu_{i} \nabla_{w^{T}} J_{i}\left(\phi_{i, k-1}\right)
\end{array}\right.
$$

Where $w_{i, k}$ is the $i^{\text {th }}$ agent's estimate of $w$ at time $k$, and $\nabla_{w^{\mathrm{T}}} J_{i}\left(\phi_{i, k-1}\right)$ is the gradient of agent i's cost function calculated with the intermediate variable $\phi_{i}$; all other parameters are the same as Eq. 5.2.1. The weights will also remain the same Metropolis values as with information sharing; this guarantees stability, good performance and the ability to adapt to changing topologies.

With distributed optimization through diffusion, not all agents need to be informed, or have an associated cost function [8]. As is the case with the proposed distributed MG optimization, only DGA, GA and ESA have a gradient for the second term of Eq. 5.3.5; all others are uninformed. However, these uninformed agents still have a purpose in 
disseminating the information across the network by implementing the first term of Eq. 5.3.5.

As explained in [35], although a similar strategy exists for consensus communication, which is implemented in [20], said strategy operates over two separate time-scales: one for collecting data and another for iterating over the data. This process delays adaptation; on the other hand, diffusion processes the data in real time as it arrives to the nodes. Additionally, stability in the consensus optimization strategy is not guaranteed because of an asymmetry in its formulation. As established by the authors in [35] and therein cited works, diffusion provides improved stability, lower mean-square error and faster convergence than consensus. For these reasons, the diffusion algorithm is implemented in the proposed decentralized MG control system; this has not been done in the literature thus far.

\subsection{DISTRIBUTED MICROGRID OPTIMIZATION}

The goal of having a decentralized control system for a Microgrid (MG) is achieved through the use of diffusion by combining both information sharing and distributed optimization, and adjusting them to a new scheme. The $J$ functions described in Eq. 5.3.1 correspond to the cost functions associated with the dispatchable resources, namely the Distributed Generator Agent (DGA), Grid Agent (GA), and Energy Storage Agent (ESA). Recalling these functions from previous sections:

$$
\begin{gathered}
F_{D G}=a+b P_{D G}+c P_{D G}^{2} \\
\frac{d F_{D G}}{d P_{D G}}=b+2 c P_{D G}
\end{gathered}
$$




$$
\begin{gathered}
P_{D G, \text { min }} \leq P_{D G} \leq P_{D G, \max } \\
F_{G}=r_{u} \cdot P_{G} \\
\frac{d F_{G}}{d P_{G}}=r_{u} \\
F_{B}=a+b\left(P_{B}+3 P_{B, \max }(1-S O C)\right)+c\left(P_{B}+3 P_{B, \max }(1-S O C)\right)^{2} \\
\frac{d F_{B}}{d P_{B}}=b+c\left(2 P_{B}-6 P_{B, \max }(S O C-1)\right) \\
-P_{B, \max } \leq P_{B} \leq P_{B, \max }
\end{gathered}
$$

The vector of arguments $w$ has an entry for each of the agents in the network, however only the dispatchable resources have a nonzero value; all other nodes on the network remain at zero and are not updated. Thus, continuing the case presented in Section 5.1 and illustrated in Fig. 5.1.1, the $w$ vector would be:

$$
w=\left[\begin{array}{c}
P_{B} \\
0 \\
P_{D G} \\
0 \\
0 \\
P_{G}
\end{array}\right]
$$

Where $P_{B}, P_{D G}$ and $P_{G}$ are the power from the ESA, DGA and GA, respectively. Note that each agent has its own approximation of the vector $w_{i}$ which it updates and diffuses according to Eq. 5.3.5. On the other hand, for the estimation problem described in Eq. 5.2.1, the approximation $x_{i}$ is a scalar value representing the average power required in the system. 
Because each agent has unique knowledge of its own resource, the cost function and gradient for dispatchable agent $i$ are only defined for the $i^{\text {th }}$ element of vector $w$; all other elements of these functions being zero:

$$
\begin{gathered}
J_{i}\left(w_{i}\right)=F_{i}\left(w_{i}[i]\right) \\
\nabla J_{i}\left(w_{i}\right)=\nabla F_{i}\left(w_{i}[i]\right)
\end{gathered}
$$

Thus, the gradient update step in the second term of Eq. 5.3.5 only affects the agent's own power level (when such a gradient exists). Intuitively, the gradient acts by “nudging” an agent's power level towards a minimum. Because these nudges are being transmitted through diffusion, the agents with the larger gradient (and hence the larger marginal cost) will be nudged further down than the others. Consequently, the updating parameter $\mu_{i}$ must be the same for every agent in order to reach the Economic Dispatch (ED) point. As mentioned in Section 5.2, a desirable side effect of having the updating parameter $\mu_{i}$ be unchanged throughout optimization is that the global minimum is tracked as conditions change. In fact, this is one of the key features of this decentralized optimization scheme and will be demonstrated in Chapter 6.

This concept of "nudging" is similar to the one described in Section 3.1 with the Lagrange multiplier; indeed, each dispatchable agent's gradient term will converge to this point. However, an additional step in the updating procedure must be included for the MG optimization system. The net power demand (loads minus Renewable Energy Sources (RES)) must be supplied among the dispatchable agents; this is where the information sharing and optimization diffusion methods meet. It was seen in Section 5.2 how diffusion 
can be used to broadcast the net power needed by the system; thus two separate diffusion processes are implemented:

1. The first uses the information sharing formulation of diffusion (Eq. 5.2.1) to broadcast the power being demanded by the Load Agents (LA) and provided by the Renewable Agents (RA), and all agents on the network reach an agreement on this. This happens continually and restarts after a certain precision level has been reached among all agents. Thus, the net power is constantly being tracked and diffused across the network; with modern communication infrastructures, this can happen several times a second.

2. The second uses the decentralized optimization formulation of diffusion (Eq. 5.3.5) to adjust the dispatchable resources to their ED point. This is also done continuously, as the net power demand is tracked by the first process. The net power is included in the optimization formulation in the following way:

$$
\begin{gathered}
\sum_{i=1}^{n} w[i]=P_{N} \\
P_{N}=\Sigma P_{L A}-\Sigma P_{R A}
\end{gathered}
$$

Where $P_{N}$ is the net power demand, which is defined by the addition of all loads minus all RES output. Fig. 5.4.1 describes the two-level diffusion process: 


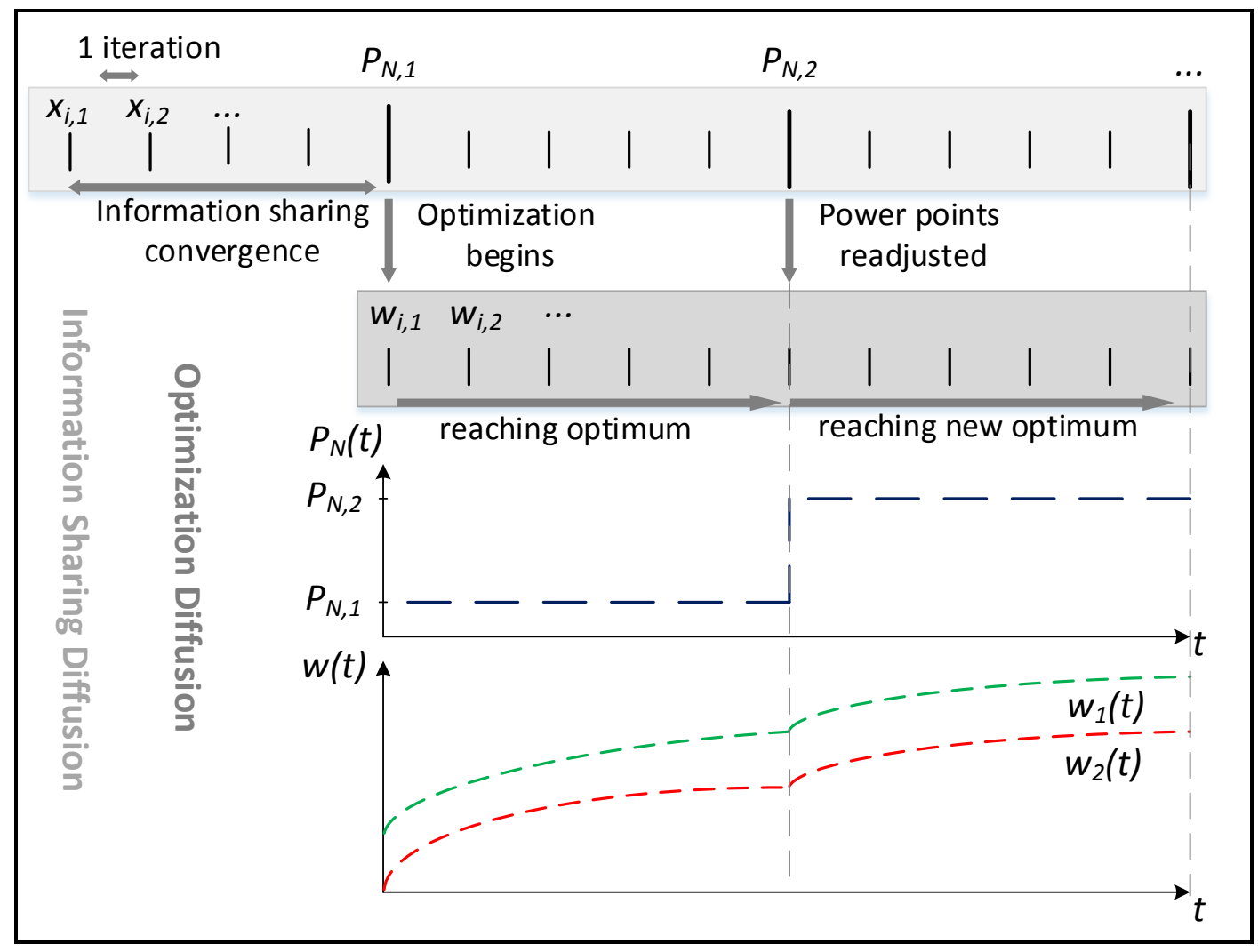

Figure 5.4.1: Two-Step Diffusion Methodology

To keep the equality in Eq. 5.4.4 after the gradient update by the dispatchable agents in Eq. 5.3.5, the "nudge" by the agent being updated is added to the other dispatchable agents plus any deficit compared to the net power:

$$
\begin{gathered}
\Delta w_{i, k}[i]=-\mu_{i} \nabla_{w^{\mathrm{T}}} J_{i}\left(\phi_{i, k-1}\right) \\
\sum_{j=1}^{n} \Delta w_{i, k}[j]=P_{N}-\sum w_{i, k-1}-\Delta w_{i, k}[i] \\
\text { where } \mathrm{j} \neq \mathrm{i} \text { and } \mathrm{j} \in \mathrm{GA}, \mathrm{DGA}, \mathrm{ESA}
\end{gathered}
$$

Where $\Delta w_{i, k}[i]$ is the so called gradient nudge of Eq. 5.3.5, and so Eq. 5.4.7 describes how this nudge is distributed among the other dispatchable agents to get to the net power demand. This is a key variance of the diffusion methods investigated; without it, 
the power points would drift to each agent's minimum cost and the power balance would not be maintained. Fig 5.4.2 shows this concept visually:

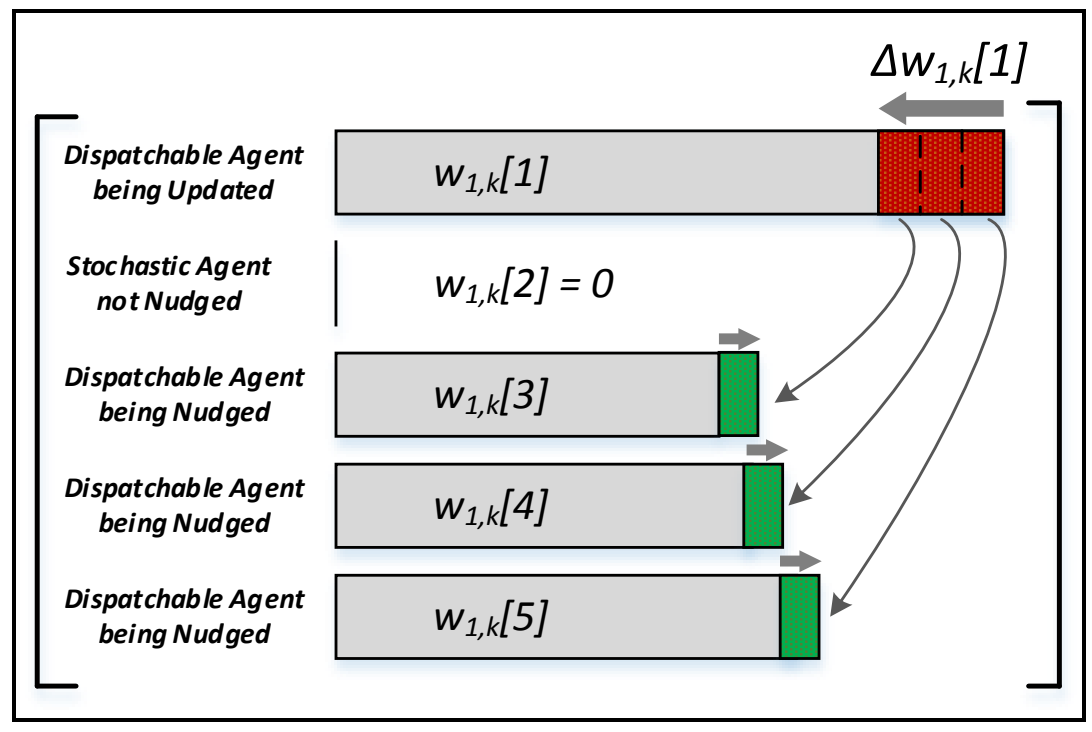

Figure 5.4.2: Nudge Methodology

To test the concept as in the previous sections, the same MG example depicted in Fig. 5.1.1 is used for CTA Optimization. For simplicity, the BESS is substituted with another DG and thus we have the first DG on node one (DG1) and the second on node three (DG2), with the following cost and marginal cost functions:

$$
\begin{gathered}
F_{D G 1}=30+4 e-8 P_{D G 1}+6 e-11 P_{D G 1}^{2} \\
\frac{d F_{D G 1}}{d P_{D G 1}}=4 e-8+1.2 e-10 P_{D G 1} \\
0 \leq P_{D G 1} \leq 2 M W \\
F_{D G 2}=20+5 e-8 P_{D G 2}+10 e-11 P_{D G 2}^{2}
\end{gathered}
$$




$$
\begin{aligned}
\frac{d F_{D G 2}}{d P_{D G 2}} & =5 e-8+2 e-10 P_{D G 2} \\
0 & \leq P_{D G 2} \leq 1.5 M W
\end{aligned}
$$

First, an islanded MG is analyzed, where the net power requirement adds up to 2.4 MW; using the Lagrangian method as described in Section 3.1, the following ED point is reached:

Table 5.4.1: Islanded MG ED

\begin{tabular}{|c|c|c|c|c|c|c|c|c|}
\hline $\begin{array}{c}\text { Total } \\
\text { Power } \\
\text { (W) }\end{array}$ & $\begin{array}{c}\text { Power } \\
\text { DG1 (W) }\end{array}$ & $\begin{array}{c}\text { Power } \\
\text { DG2 (W) }\end{array}$ & $\begin{array}{c}\text { Marginal } \\
\text { Cost } \\
\text { DG1 }\end{array}$ & $\begin{array}{c}\text { Marginal } \\
\text { Cost } \\
\text { DG2 }\end{array}$ & $\begin{array}{c}\text { Lagrange } \\
\text { Multiplier } \\
(\mathbf{A})\end{array}$ & Cost DG1 & Cost DG2 & Total Cost \\
\hline $2.4 \mathrm{E}+6$ & $1.5 \mathrm{E}+6$ & $9 \mathrm{E}+5$ & $1.8 \mathrm{E}-4$ & $1.8 \mathrm{E}-4$ & $1.8 \mathrm{E}-4$ & $\$ 165.07$ & $\$ 101.04$ & $\$ 266.11$ \\
\hline
\end{tabular}

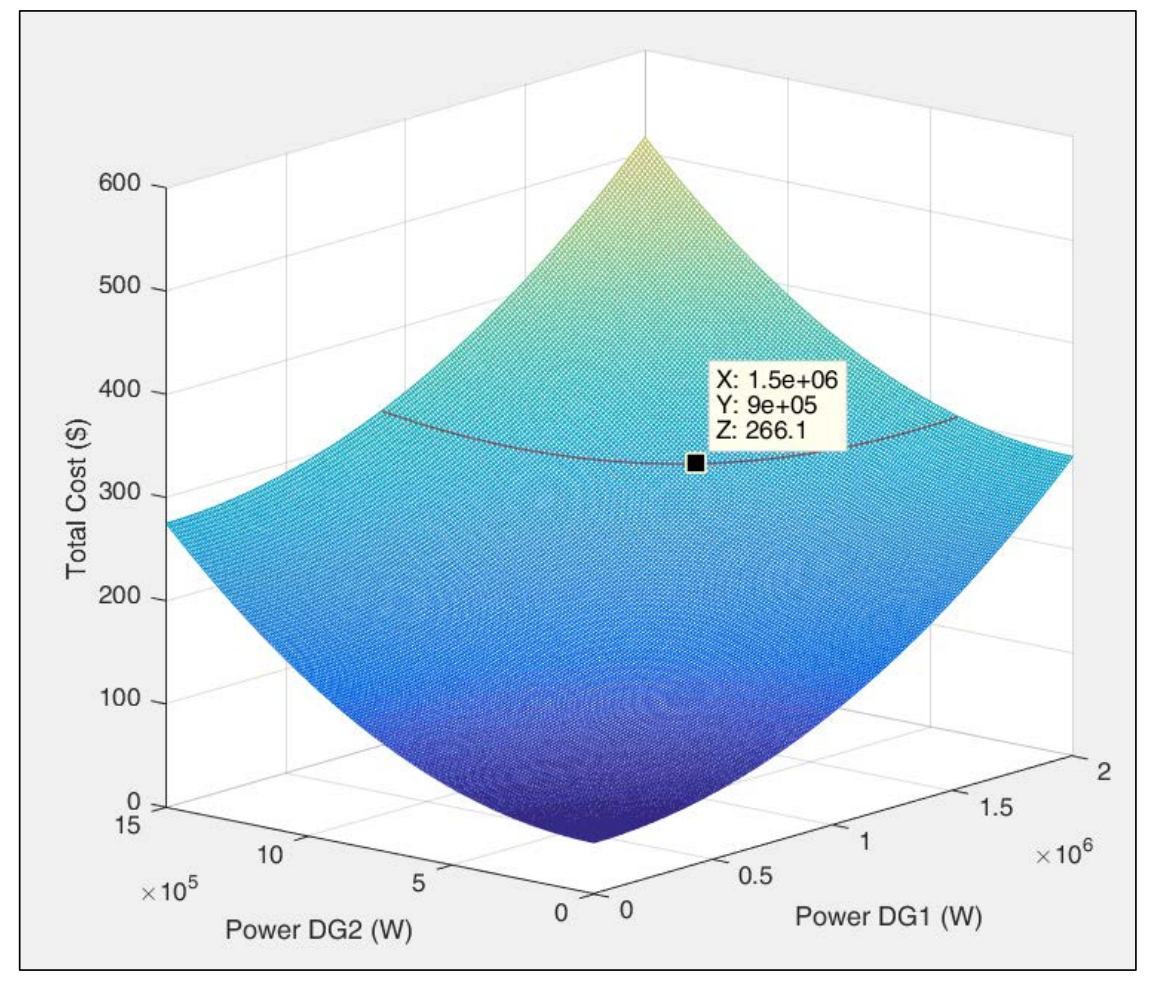

Figure 5.4.3: Islanded MG Cost Functions ED

As can be seen in the above table, the power points of the two DGs add up to the total power required and the marginal cost of each equals the Lagrange Multiplier $(\lambda)$. The 
total cost comes out to $\$ 266$ (per hour). One can visualize this by viewing both cost functions in a surface plot with the intersection line representing the total power constraint (Fig. 5.4.3).

Similarly, the marginal cost functions can each be plotted and the Lagrangian ED point would correspond to where their intersection meets the total power constraint:

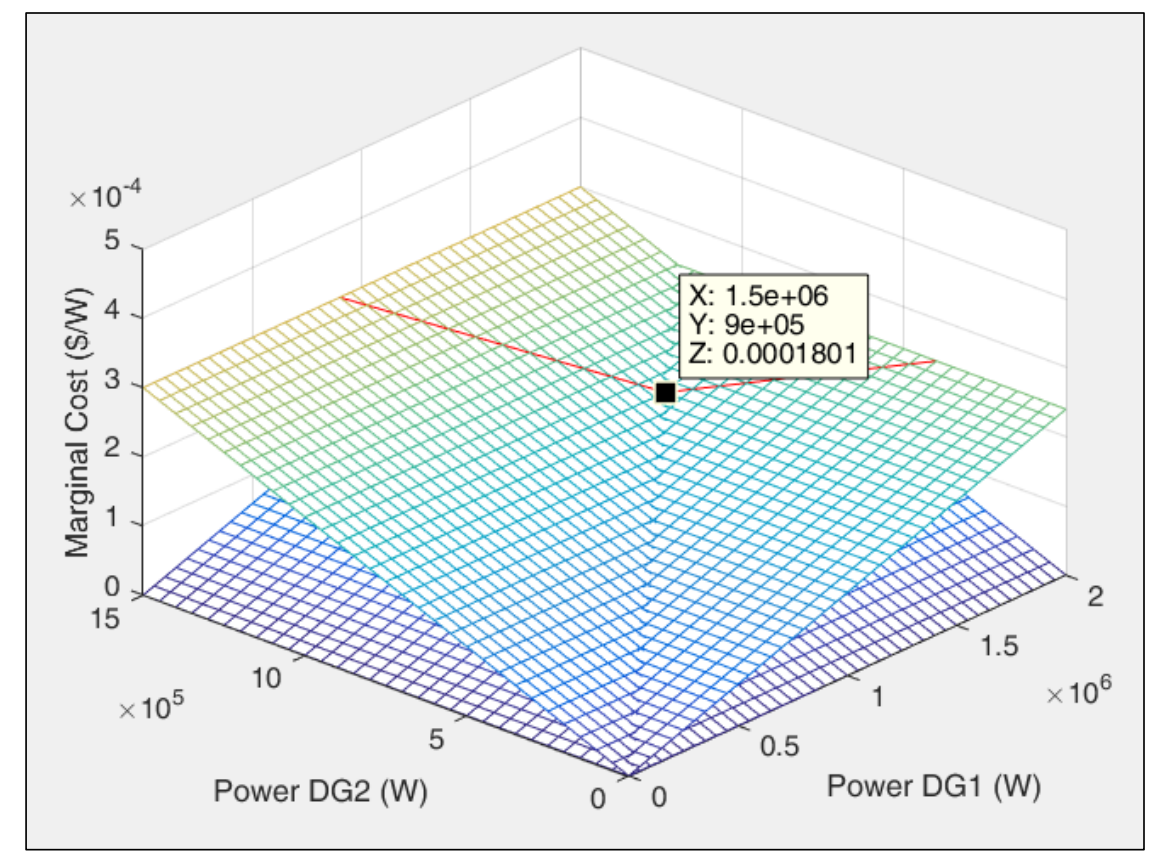

Figure 5.4.4: Islanded MG Marginal Cost Functions ED

Implementing CTA Diffusion, as described by Eq. 5.3.5, the following adaptation is observed (Fig. 5.4.5). The algorithm converges at 1.493 MW for DG1 and $903 \mathrm{~kW}$ after 2,000 iterations with an updating parameter $\mu$ of 1e8; the total adds up to $2.396 \mathrm{MW}$, which is just $0.17 \%$ below the actual total. The ED points are also quite close: $99.54 \%$ accuracy for DG1 and 99.68\% for DG2.

The discrepancy is due to the aforementioned tradeoff between accuracy and adaptability. In an actual system, these 2,000 iterations would take a couple of minutes to 


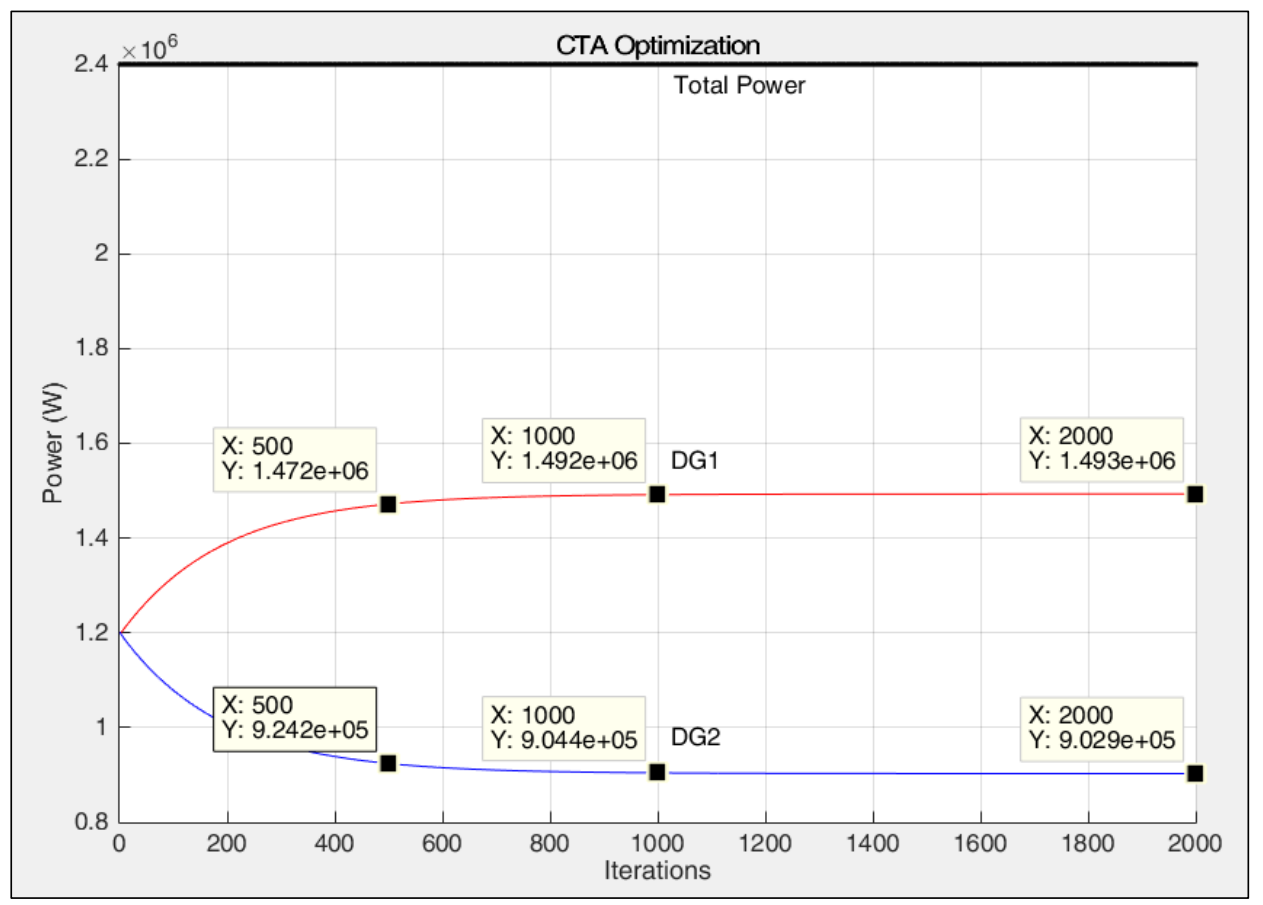

Figure 5.4.5: Islanded MG CTA Diffusion, $\mu=1 e 8$

reach, which is acceptable, taking into account the optimization of the power sources is ongoing over a 24-hour period. However, at only 1,000 iterations, the accuracy was quite comparable at around 99.5\%, and at 500 iterations, it was already around 98\%. If more adaptability and faster tracking is desired, the $\mu$ can be increased.

With the updating parameter $\mu$ four times as large (Fig. 5.4.6), the convergence is much quicker, but the accuracy suffers; after 500 iterations the accuracy is just over $98 \%$. With only 250 iterations the accuracy is already at almost 98\%, and at 100 iterations at around 95\%. An interesting point to note is that after 500 iterations this larger $\mu$ reaches a higher accuracy than after 500 iterations with the smaller $\mu$ (1.474 vs 1.472$)$; therefore the $\mu$ must be adjusted according to the scenario and desired accuracy. Further increasing the updating parameter, this trend is continued (Fig. 5.4.7). 


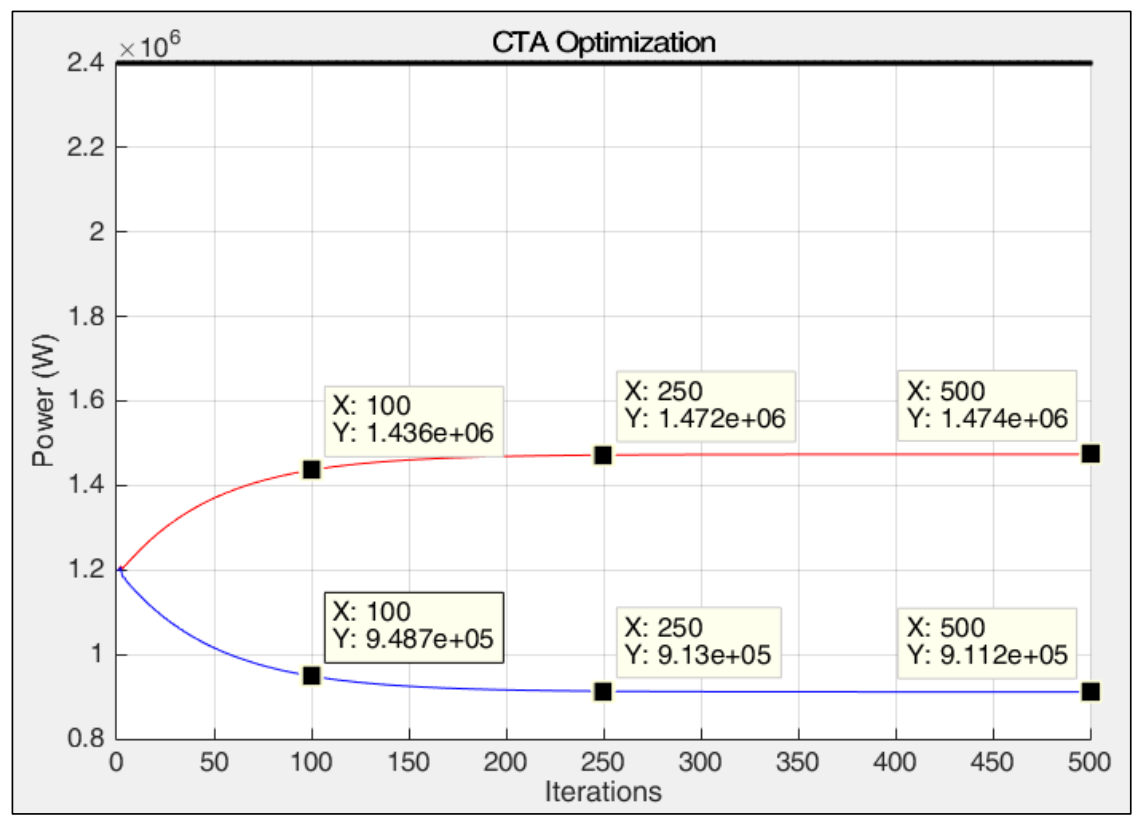

Figure 5.4.6: Islanded MG CTA Diffusion, $\mu=4 e 8$

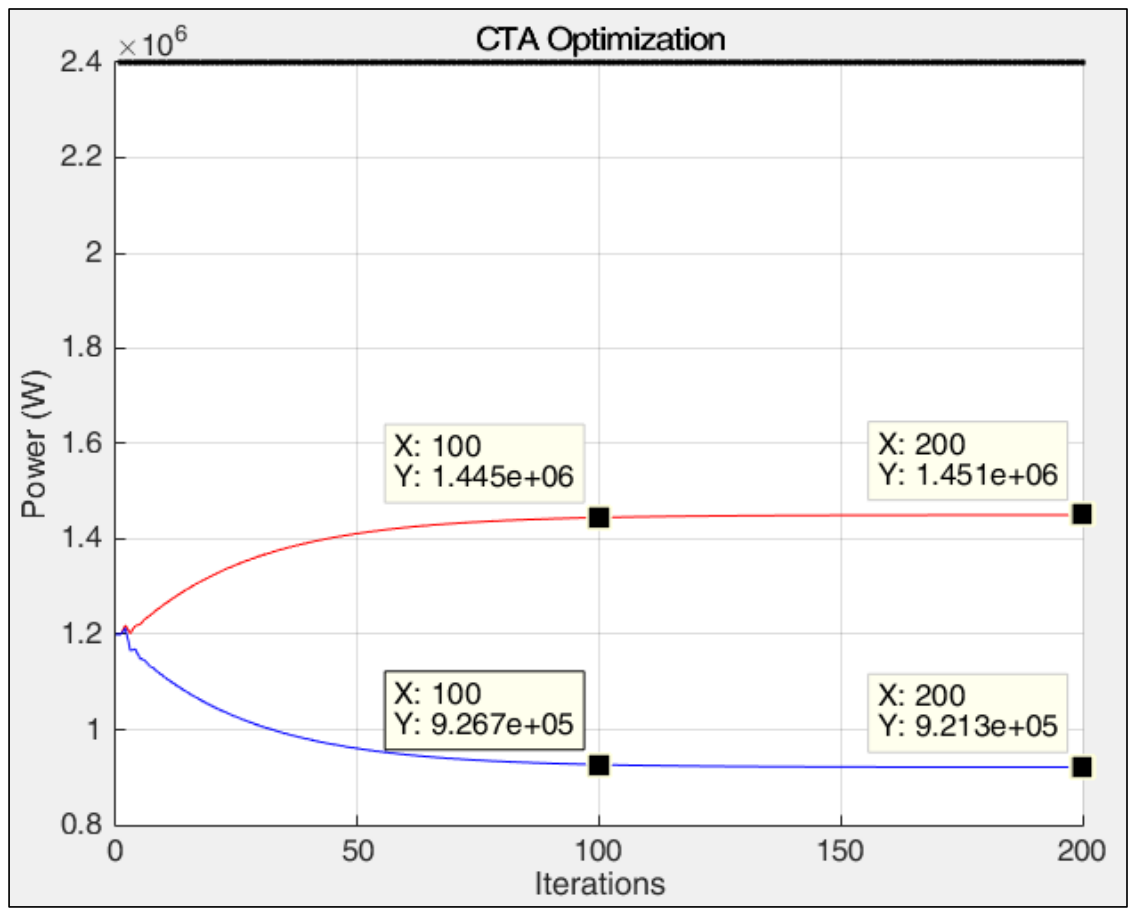

Figure 5.4.7: Islanded MG CTA Diffusion, $\mu=8 e 8$ 
Now the accuracy really suffers, around 97\% after 200 iterations and 96\% after 100 iterations; but on the other, hand very quick adaptation is achieved at just 100 iterations, which would typically only take a few seconds.

When the MG is grid connected, it was seen in Section 4.3 that the Lagrange multiplier, and thus the marginal costs, would converge to the grid price. When enabling the grid with an electric rate of $\$ 0.10 / \mathrm{kWh}$ ( $\$ 1 \mathrm{e}-4 \$ / \mathrm{W}$ ), the following ED solutions is obtained:

Table 5.4.2: Grid-Connected MG ED

\begin{tabular}{|c|c|c|c|c|c|c|c|c|c|c|c|}
\hline $\begin{array}{c}\text { Total } \\
\text { Power } \\
\text { (W) }\end{array}$ & $\begin{array}{l}\text { Power } \\
\text { DG1 } \\
\text { (W) }\end{array}$ & $\begin{array}{l}\text { Power } \\
\text { DG2 } \\
\text { (W) }\end{array}$ & $\begin{array}{l}\text { Power } \\
\text { Grid } \\
\text { (W) }\end{array}$ & $\begin{array}{l}\text { Grid } \\
\text { Price } \\
\text { (\$/W) }\end{array}$ & \begin{tabular}{|c|} 
Marginal \\
Cost \\
DG1
\end{tabular} & $\begin{array}{c}\text { Marginal } \\
\text { Cost } \\
\text { DG2 }\end{array}$ & $\begin{array}{c}\text { Lagrange } \\
\text { Multiplier } \\
(\lambda)\end{array}$ & $\begin{array}{l}\text { Cost } \\
\text { DG1 }\end{array}$ & $\begin{array}{l}\text { Cost } \\
\text { DG2 }\end{array}$ & $\begin{array}{l}\text { Cost } \\
\text { Grid }\end{array}$ & $\begin{array}{l}\text { Total } \\
\text { Cost }\end{array}$ \\
\hline $2.4 \mathrm{E}+6$ & $8.33 E+5$ & $5 E+5$ & $1.07 \mathrm{E}+6$ & $1 E-4$ & $1 \mathrm{E}-4$ & $1 \mathrm{E}-4$ & $1 \mathrm{E}-4$ & $\$ 71.67$ & $\$ 45.00$ & $\$ 85.38$ & $\$ 202.05$ \\
\hline
\end{tabular}

As expected, the total cost decreases because the utility electricity rate is below the marginal cost of the islanded case ( $\$ 0.10 / \mathrm{kW}$ vs $\$ 0.18 / \mathrm{kW}$ ). Including the Grid Agent (GA) in the CTA Diffusion optimization, the following is observed (Fig. 5.4.8). With the same updating parameter $\mu$ and number of iterations as in the first case of the islanded MG, a total of 2.393 MW (99.72\% of total) is reached with around 97\% accuracy on the individual power points.

Obviously, the initial estimates affect the convergence time; because this process will be ongoing, the previous estimate will be used as a starting point for the next iteration of the optimization and so it will track the solution more closely.

This can be seen in action by starting with closer estimates under the same circumstances, which reach over 98\% accuracy (Fig. 5.4.9). 


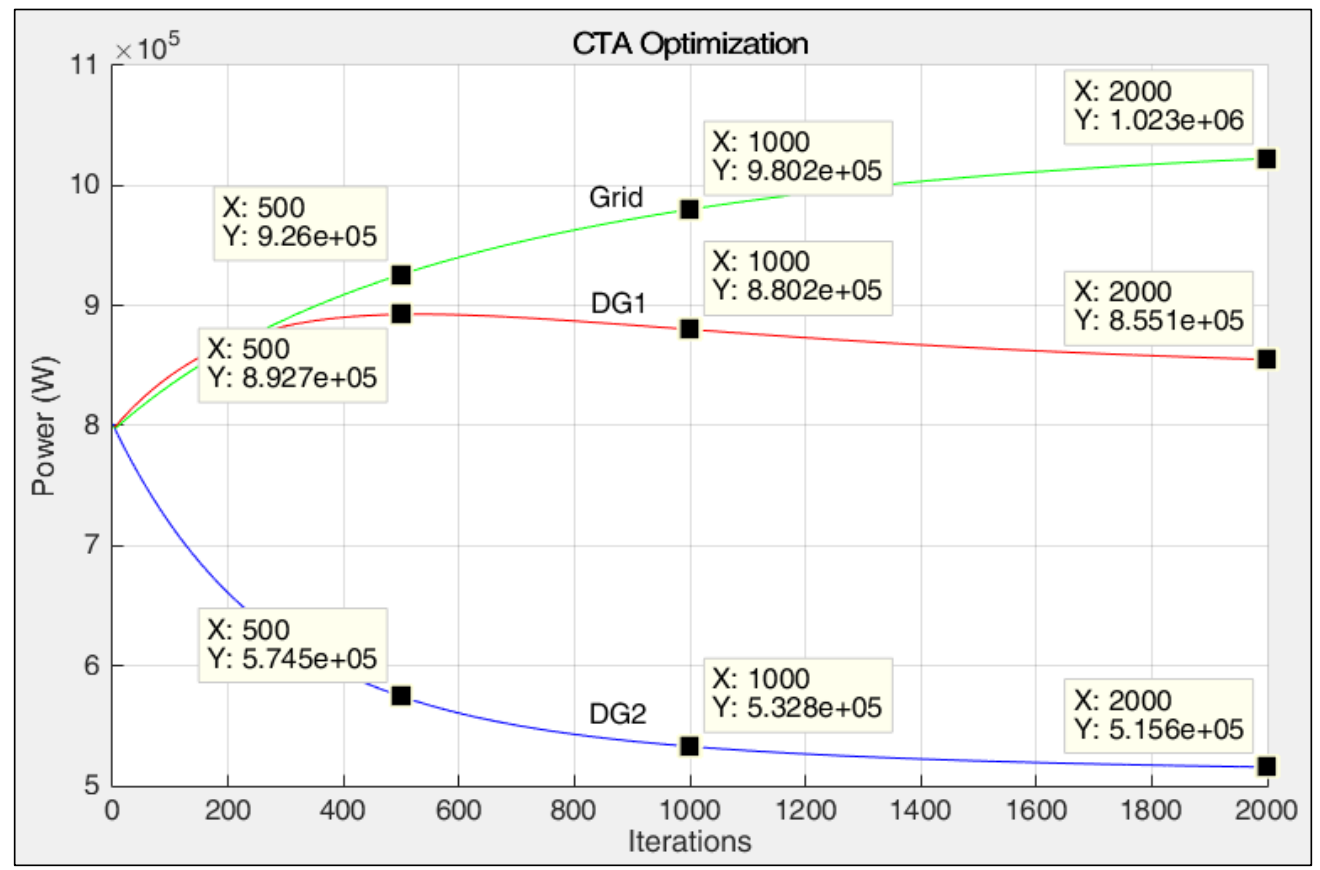

Figure 5.4.8: Grid-Connected MG CTA Diffusion, $\mu=1 e 8$

Additional to the net power constraints, the dispatchable agents have unique constraints on the minimum and maximum power of their corresponding resource. In [36],

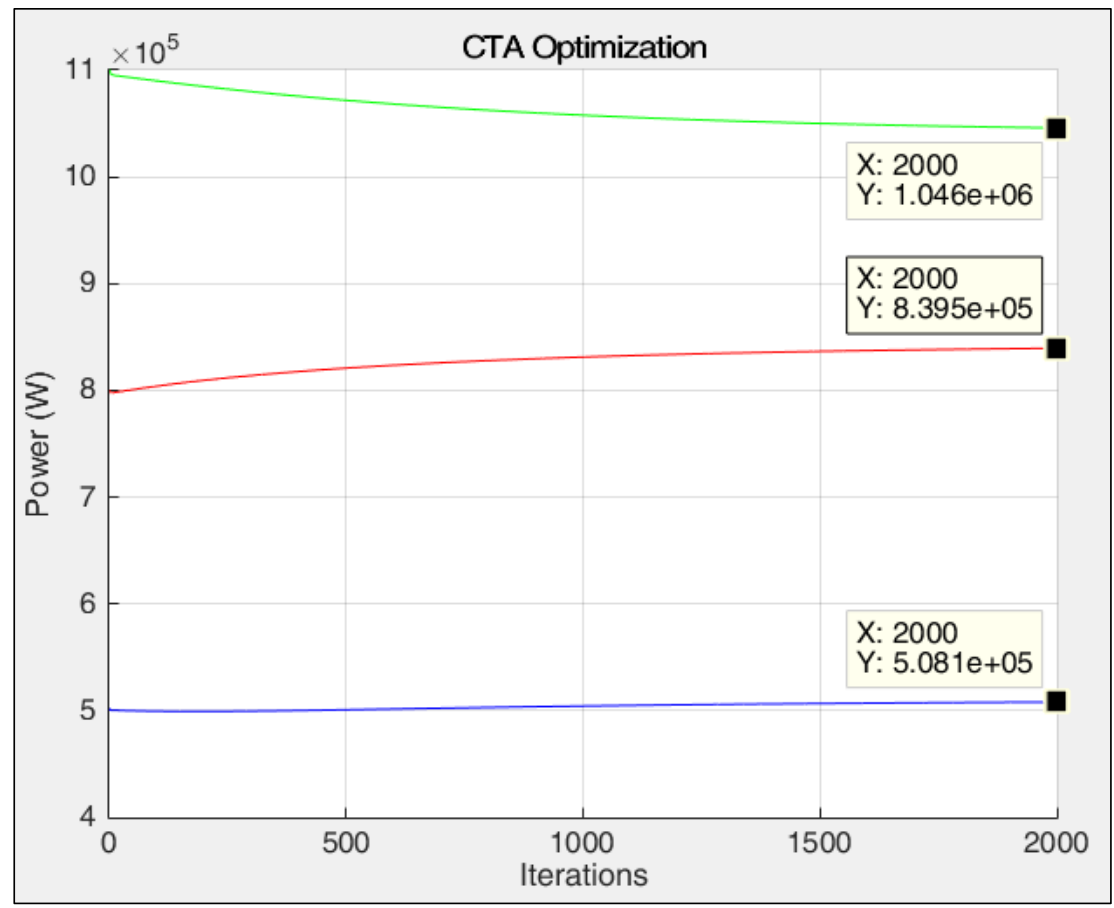

Figure 5.4.9: Grid-Connected MG CTA Diffusion, $\mu=1 e 8$ 
the authors include a barrier or penalty function around the feasible region. The penalty method is preferred, as it maintains the continuity of the cost function and can converge to a solution even if the initial or intermediate states fall beyond the constraints. The objective described by Eq. 5.4.2 becomes:

$$
\begin{gathered}
\min _{w} J_{g l o b}(w)+\eta \sum_{i=1}^{n} \delta_{i}\left(w_{i}\right) \\
\delta_{i}\left(w_{i}\right)=\left\{\begin{array}{cc}
0 & w_{i, \min } \leq w_{i} \leq w_{i, \text { max }} \\
\alpha\left(w_{i}-w_{i, \max }\right)^{2} & w_{i}>w_{i, \text { max }} \\
\alpha\left(w_{i}-w_{i, \min }\right)^{2} & w_{i}<w_{i, \text { min }}
\end{array}\right.
\end{gathered}
$$

Where $\eta$ is a parameter which dictates the importance of obeying the constraints, and $\delta$ is the penalty function defined for each element in $w$ according to its inequality constraints. Eq. 5.4.15 describes a simple addition to the cost function of the squared deviation from the constraint tuned by scalar $\alpha$; Fig. 5.4.10 adds this penalty function to the same fossil fuel generator described in Section 3.1 and illustrated in Fig. 3.1.1.

Notice that the cost function maintains its convexity; in fact, in the case of the quadratic cost function for the distributed generators, the function remains strongly convex. The marginal cost demonstrates how the optimization method would behave if an initial or intermediate state would lie beyond the constraints (Fig. 5.4.11). 


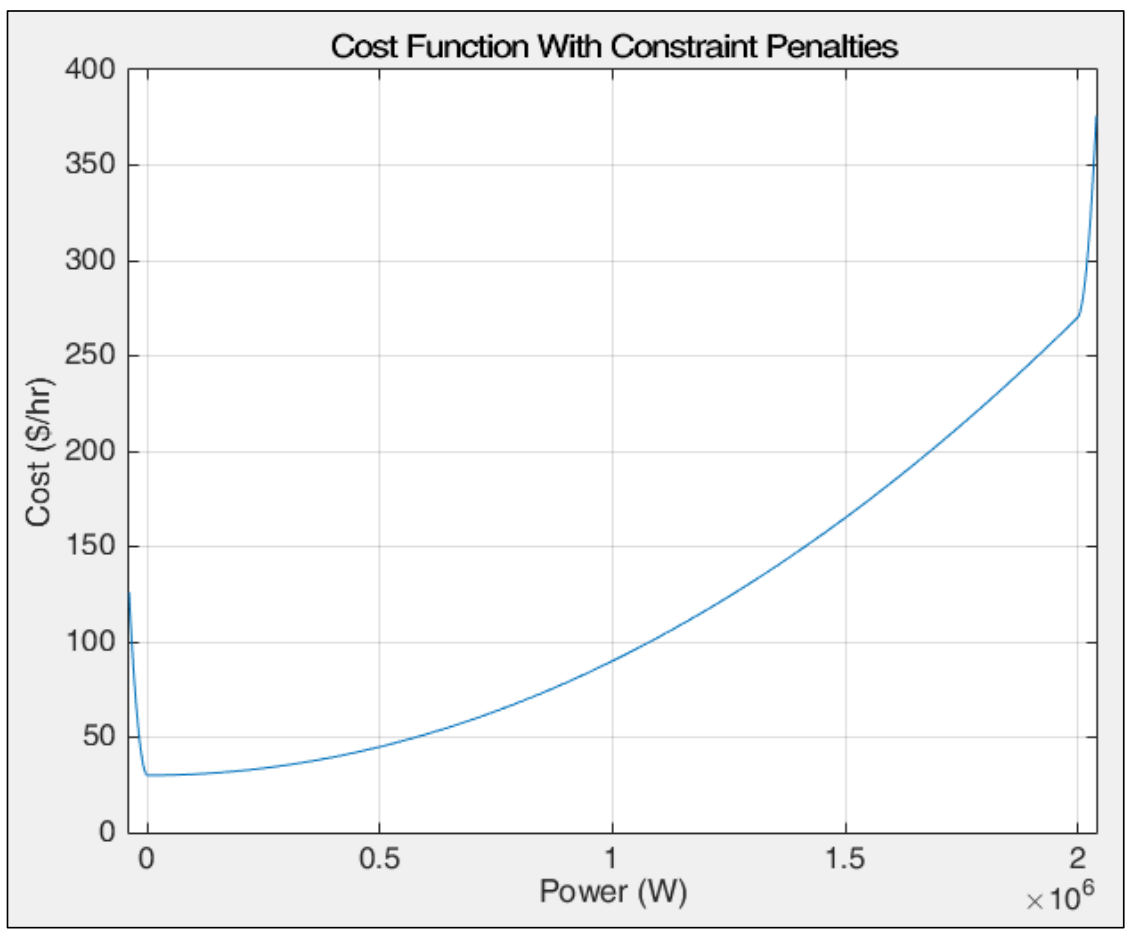

Figure 5.4.10: Fossil Fuel Generator Cost Function with Constraint Penalties

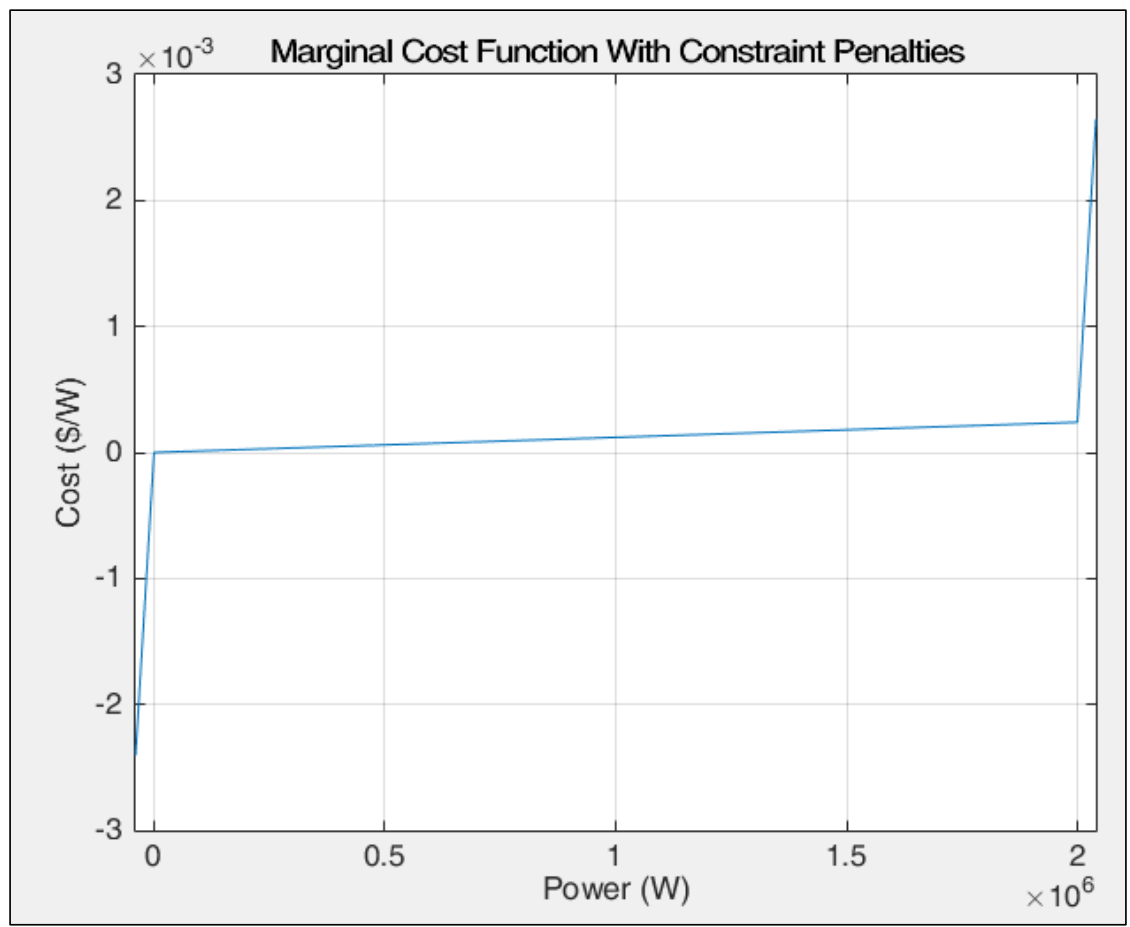

Figure 5.4.11: Fossil Fuel Generator Marginal Cost Function with Constraint Penalties 
As can be seen in Fig. 5.4.11, if at any stage an estimate for an argument $w_{i}$ [i] falls above the maximum power constraint, the derivative inflates so rapidly it would quickly "nudge" the estimate back towards the valid zone. Likewise, below the minimum power, the gradient would be negative, which would swiftly shift the estimate back up to within the constraints. Moreover, the Lagrangian point would never settle in negative values for the system being described.

Adding these constraints to the MG being used as an example, another scenario is devised to test how the optimization behaves at the constraint borders. Suppose the grid price shot up to $\$ 0.28 / \mathrm{kWh}$ due to a high system peak and the net MG demand was $3 \mathrm{MW}$ :

Table 5.4.3: Grid-Connected MG Peak ED

\begin{tabular}{|c|c|c|c|c|c|c|c|c|c|c|c|}
\hline $\begin{array}{l}\text { Total } \\
\text { Power } \\
\text { (W) }\end{array}$ & $\begin{array}{l}\text { Power } \\
\text { DG1 (W) }\end{array}$ & $\begin{array}{l}\text { Power } \\
\text { DG2 } \\
\text { (W) }\end{array}$ & $\begin{array}{l}\text { Power } \\
\text { Grid (W) }\end{array}$ & $\begin{array}{l}\text { Grid } \\
\text { Price } \\
\text { (\$/W) }\end{array}$ & \begin{tabular}{|l} 
Marginal \\
Cost \\
DG1
\end{tabular} & \begin{tabular}{|l} 
Marginal \\
Cost \\
DG2
\end{tabular} & \begin{tabular}{|c|} 
Lagrange \\
Multiplier \\
$(\lambda)$
\end{tabular} & $\begin{array}{l}\text { Cost } \\
\text { DG1 }\end{array}$ & $\begin{array}{l}\text { Cost } \\
\text { DG2 }\end{array}$ & $\begin{array}{l}\text { Cost } \\
\text { Grid }\end{array}$ & $\begin{array}{l}\text { Total } \\
\text { Cost }\end{array}$ \\
\hline $3 E+6$ & $2.333 E+6$ & $1.4 \mathrm{E}+6$ & $-7.33 E+5$ & $2.8 \mathrm{E}-4$ & $2.8 \mathrm{E}-4$ & $2.8 \mathrm{E}-4$ & $2.8 \mathrm{E}-4$ & $\$ 356.67$ & $\$ 216$ & $-\$ 205.2$ & $\$ 367.5$ \\
\hline
\end{tabular}

As can be seen, the price is so high that the MG starts feeding power back the grid; however, this scenario violates the power constraint on DG1, which is 2MW (Eq. 5.4.10). Therefore, the ED needs to be re-done with DG1 at its maximum:

Table 5.4.4: Grid-Connected MG Peak ED w/Constraints

\begin{tabular}{|c|c|c|c|c|c|c|c|c|c|c|c|}
\hline $\begin{array}{l}\text { Total } \\
\text { Power } \\
\text { (W) }\end{array}$ & $\begin{array}{l}\text { Power } \\
\text { DG1 (W) }\end{array}$ & $\begin{array}{l}\text { Power } \\
\text { DG2 } \\
\text { (W) }\end{array}$ & $\begin{array}{l}\text { Power } \\
\text { Grid (W) }\end{array}$ & $\begin{array}{l}\text { Grid } \\
\text { Price } \\
\text { (\$/W) }\end{array}$ & \begin{tabular}{|c|} 
Marginal \\
Cost \\
DG1
\end{tabular} & $\begin{array}{c}\text { Marginal } \\
\text { Cost } \\
\text { DG2 }\end{array}$ & $\begin{array}{l}\text { Lagrange } \\
\text { Multiplier } \\
(\Lambda)\end{array}$ & $\begin{array}{l}\text { Cost } \\
\text { DG1 }\end{array}$ & $\begin{array}{l}\text { Cost } \\
\text { DG2 }\end{array}$ & $\begin{array}{l}\text { Cost } \\
\text { Grid }\end{array}$ & $\begin{array}{l}\text { Total } \\
\text { Cost }\end{array}$ \\
\hline $3 \mathrm{E}+6$ & $2 E+6$ & $1.4 \mathrm{E}+6$ & $-4 \mathrm{E}+5$ & $2.8 \mathrm{E}-4$ & $2.4 E-4$ & $2.8 \mathrm{E}-4$ & $2.8 \mathrm{E}-4$ & $\$ 270$ & $\$ 216$ & $-\$ 111.9$ & $\$ 374.2$ \\
\hline
\end{tabular}

DG1 is maintained at its maximum with a lower marginal cost than the system's $\lambda$, and as can be seen, when observing the power constraints, the total cost increases because 
the assets are not operating at their optimum levels. Using the constraint penalties for the grid-connected MG scenario, the following behavior is observed:

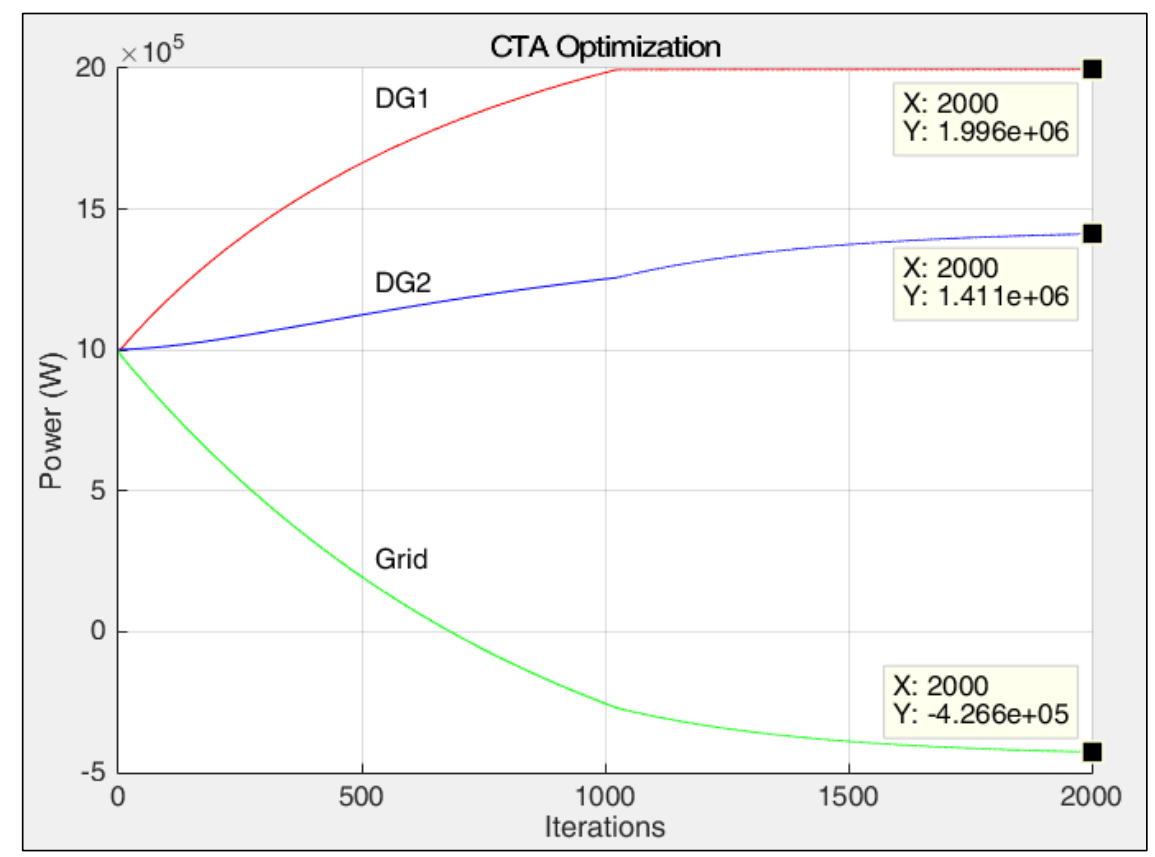

Figure 5.4.12: Grid-Connected MG CTA Diffusion w/Constraints, $\mu=1 e 8$

The CTA Optimization quickly approaches DG1's power constraint, and then stabilizes, making the other assets adapt more quickly towards their ED point. In this case, the accuracy for the DGs is over $99 \%$, however the Grid power point is only $93 \%$ accurate because it started so far off its optimal point.

In Chapter 6, this decentralized optimization system will be implemented in simulation and experimental platforms to assess its performance and the ability to track the optimal ED point. 


\section{CHAPTER 6: FULLY DECENTRALIZED MULTI-AGENT SYSTEM FOR OPTIMAL MICROGRID CONTROL}

Taking the background and formulations presented thus far, this chapter puts everything together into a comprehensive decentralized control system for optimal Microgrid management. This chapter gives an overview and an initial effort towards fully decentralized multi-agent systems for optimal control of microgrids. First, an overview of the control scheme will be illustrated, then the agents will be described. The simulation and experimental platforms will be outlined, followed by the results of the tests.

\subsection{CONTROL SySTEM OVERVIEW}

As described in previous chapters, the proposed distributed Microgrid (MG) control system consists of an agent for every node in the electrical network, which communicates only with neighboring nodes. This Multi-Agent System (MAS) acts as the secondary and tertiary level control of the MG. Secondary by adjusting the dispatchable assets shortly after a power imbalance is detected to maintain stability, and tertiary by tracking the minimum in the global operating costs as conditions change. Primary control is left to the dispatchable devices and does not depend on the MAS to respond. This is because by definition primary control must act instantaneously and automatically, and therefore cannot be subject to communication delays. Fig. 6.1.1 shows an abstraction of this framework for a hypothetical five-device system similar to what is described in [15]: 


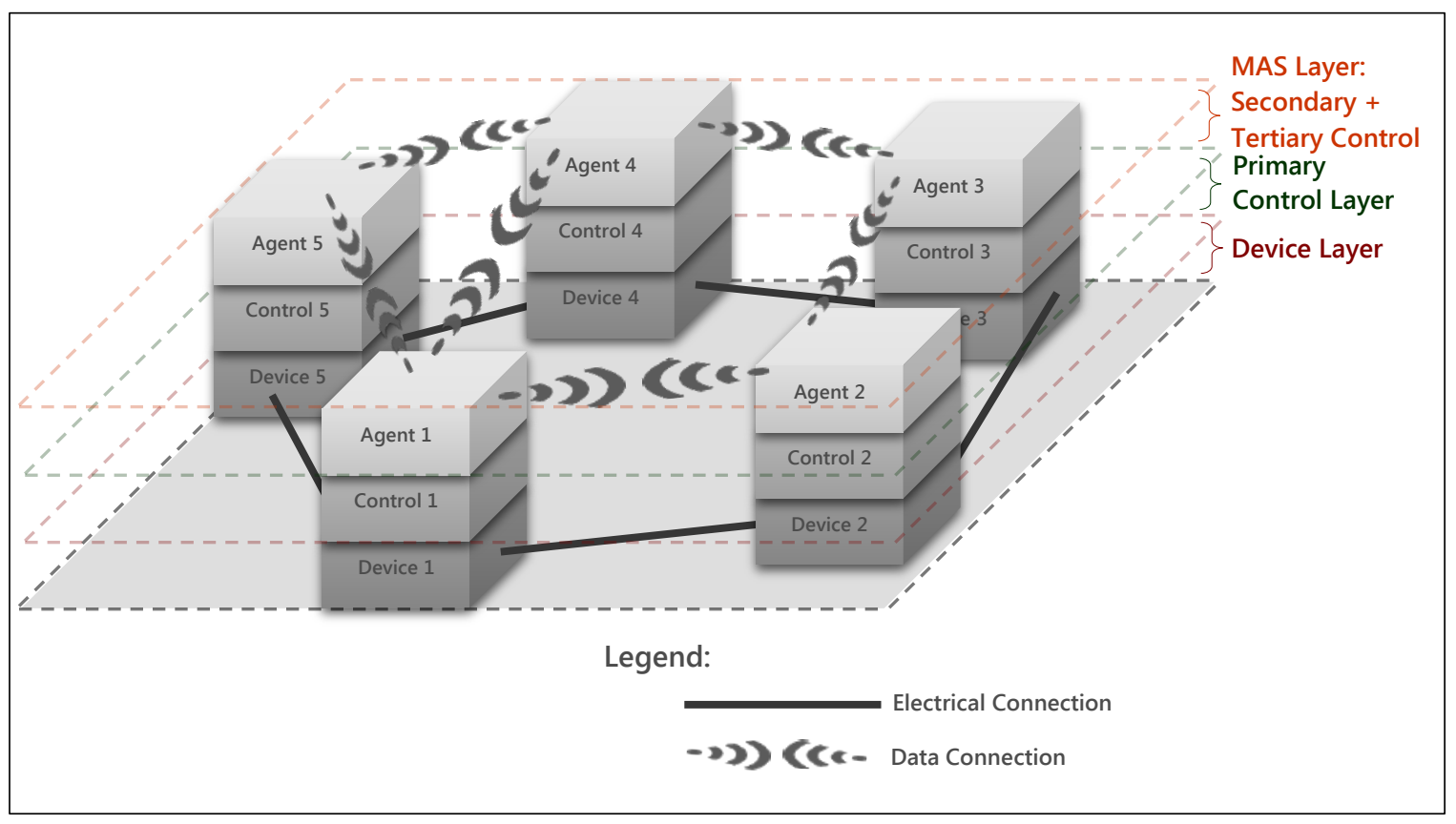

Figure 6.1.1: Control System Framework

The lower, or device, layer is where the actual electrical connections are made among the components of the MG; i.e. load feeders, Point of Common Coupling (PCC) with the utility grid and the interconnections to the three types of DERs: Renewable Energy Sources, Distributed Generators (DGs) and Energy Storage Systems.

The Primary Control layer sits atop and directly controls the corresponding devices, including any protection schemes deployed in the MG. For the PCC node, this layer measures the power in or out of the Microgrid and has control of the main breaker to make the MG islanded. For the load and RES nodes, this layer mainly measures the power being drawn or supplied and can implement protection logic and more advanced features like load shedding control and reactive power support, respectively. DER include integrated device-specific controllers such as voltage regulation and Maximum Power Point Tracking (MPPT) for solar and wind resources. Additionally, for dispatchable DERs (ESS and DGs) this layer is where droop control is located, where it has access to real time feedback 
mechanisms in order to implement the primary frequency control, as explained in Chapter 3.

The top level, termed the MAS layer, is where diffusion is implemented to provide secondary and tertiary control; this layer substitutes the centralized MG control structure. For load and RES agents, this layer gets the power measurements from the lower control level and diffuses the information to its neighbors. For DG and ESS agents, the MAS layer works to adjust the power levels of each energy source in two separate time scales: shortly after an imbalance to restore frequency, and continually to operate the resources at the global Economic Dispatch (ED) point; these are secondary and tertiary control, respectively. The operating points are communicated to the lower control levels, which adjust their corresponding device; more details on this mechanism will be described in the following sections.

\subsection{Agent Design}

There are two main types of agents, stochastic and dispatchable. The former includes Load and Renewable Agents (LA and RA, respectively), and the latter includes Grid, Distributed Generator and Energy Storage Agents (GA, DGA and ESA, respectively). The main difference is that stochastic agents measure the net power demand while the dispatchable agents control their respective resources; they can be thought of as the inputs and outputs (respectively) of a centralized control counterpart. All agents are involved in diffusing the information through the network, but only dispatchable agents have a cost function and make gradient adjustments to the diffusion optimization, as explained in Section 5.4. The main functions of the stochastic agents are illustrated in Fig. 6.2.1: 


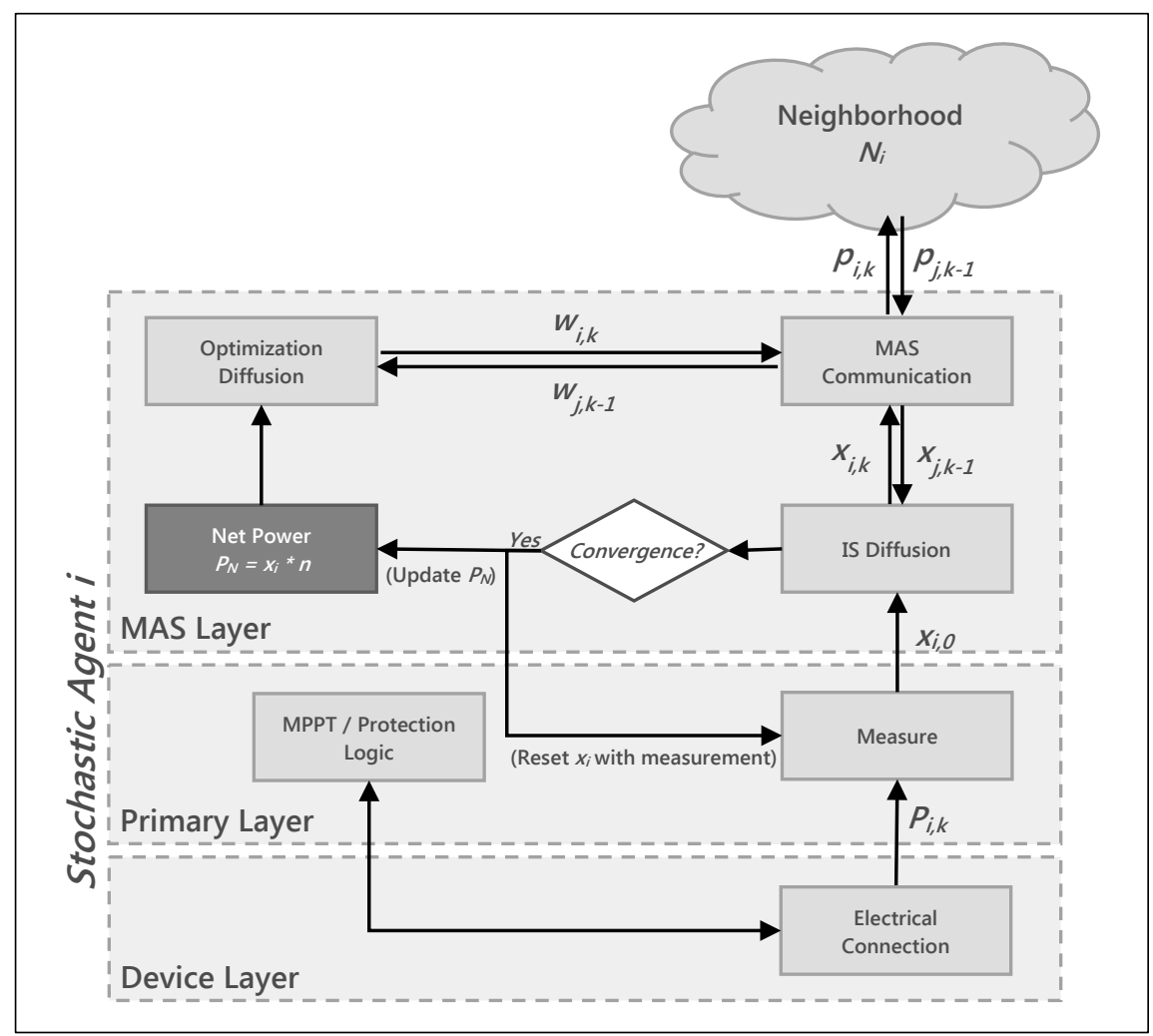

Figure 6.2.1: Stochastic Agent Block Diagram

Initially, the stochastic agents measure the electric power being fed to or from the connection being monitored, be it the load demand or the Renewable Energy Source (RES) output. Information Sharing (IS) diffusion, as described by Eq. 5.2.1, begins with the neighboring agents through the Multi-Agent System (MAS) communication framework, which will be described shortly. Once the initial convergence on $x$ (the average power demand) has been reached by all agents, the net power $P_{N}$ of the MG is established and the optimization of the DERs can commence. The agents again use the MAS communication framework to communicate their optimization estimates, $w_{i}$, to their neighbors in a combined data packet, $p_{i}$, along with the power estimate, $x_{i}$.

After the initial convergence on net power is achieved, the power estimate, $x_{i}$, is reset with the new measurement and the process restarts to reach a new agreement on the 
MG net power demand. This can happen several times a second, depending on communication bandwidths and network size and topology. Also, after the initial net power is known, the diffusion optimization continues running, indefinitely tracking the ED point for the given net power. After a new net power is agreed on, the DER power points start converging to the new ED point; this tracking continues throughout the day.

The primary layer of the stochastic agents includes the measurement devices, which read directly from the electrical connection, and the protection logic and MPPT (in case of REA), which acts bi-directionally with the device. The dispatchable agents are very similar at the highest level but differ on the primary control and device layers:

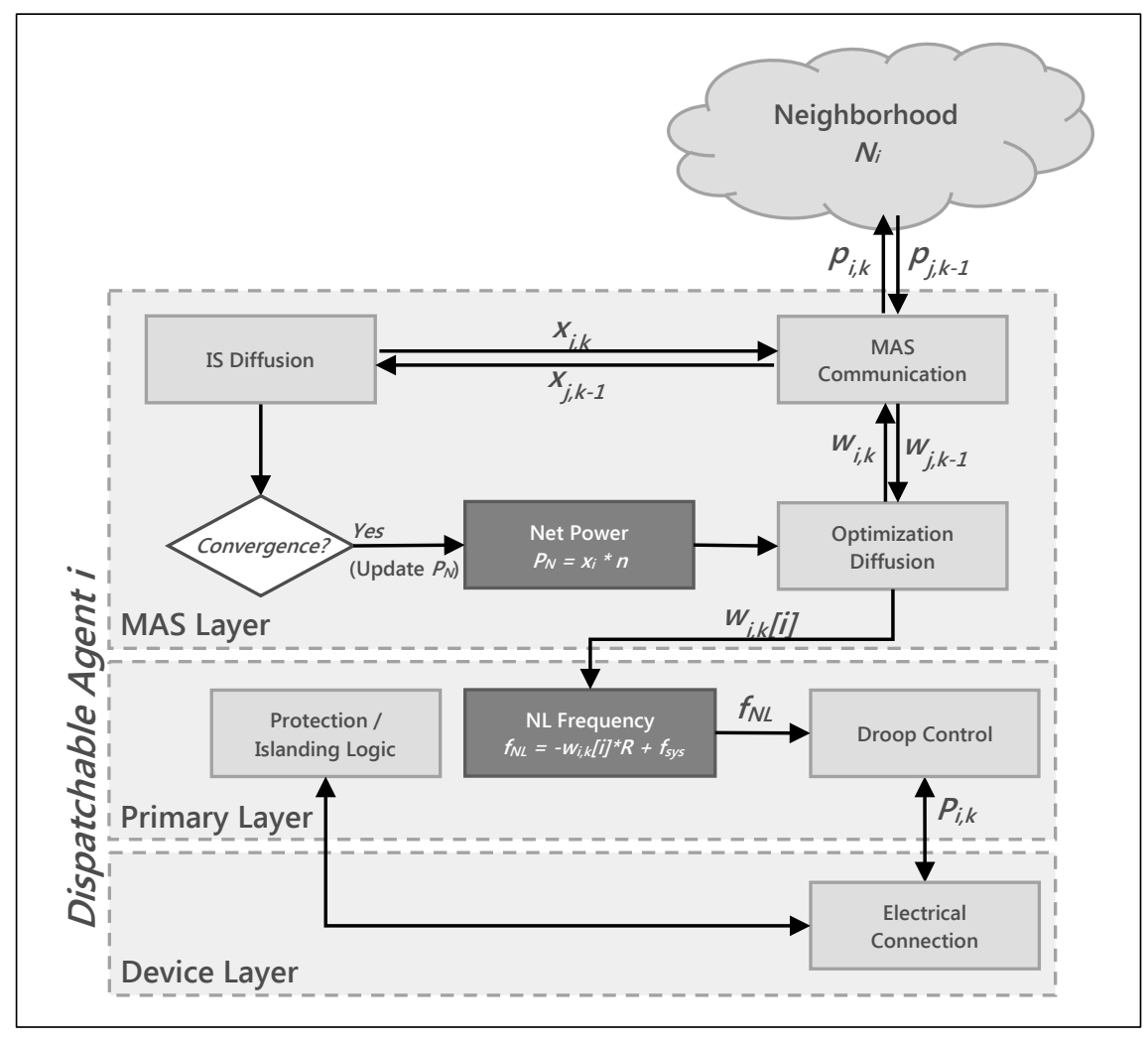

Figure 6.2.2: Dispatchable Agent Block Diagram 
As can be seen from Fig. 6.2.2, the block diagrams are quite comparable; however, notice that the Optimization and Information Sharing diffusion blocks in the MAS layer are interchanged. Further, instead of taking the measurement from the primary layer as an input, this agent updates the No-Load Frequency with the optimized self-power point, $w_{i, k}[i]$, using Eq. 3.3.8:

$$
\begin{gathered}
f_{N L}=P\left(f_{N L}-f_{F L}\right) /\left(P_{\max }-P_{\min }\right)+f_{s y s} \\
f_{N L}=-P \cdot R+f_{s y s}
\end{gathered}
$$

Where $R$ is the slope of the droop curve and the desired power point, $P$, comes from the optimized diffusion variable, $w_{i, k}[i]$ :

$$
f_{N L}=-w_{i, k}[i] \cdot R+f_{s y s}
$$

As explained in Chapter 3, this moves the droop curve up and down and controls the power output of the DER. Droop also has a feedback mechanism, as shown in Fig. 3.3.4, hence the double arrow connecting the Droop Control with the Electrical Connection block. The protection and islanding logic share this bidirectional connection as well. For the GA, which controls the islanding PCC, the droop is not implemented, as the grid will automatically adjust the power fed or drawn to match the demand and maintain the system frequency. During islanded mode, the primary and device layer for the GA will not participate at all, but the MAS layer remains active to help diffuse the information throughout the MG.

\subsection{JADE IMPLEMENTATION}

The MAS layer is deployed with the JADE Framework [37], which provides a flexible skeleton on which to customize the behavior of the different agents. Each agent 
instantiates a main class (MGAgent), which implements the IS and Optimization diffusion.

In Fig. 6.3.1, the JADE implementation similar to the one implemented in the experimental setup with five MG nodes is shown:

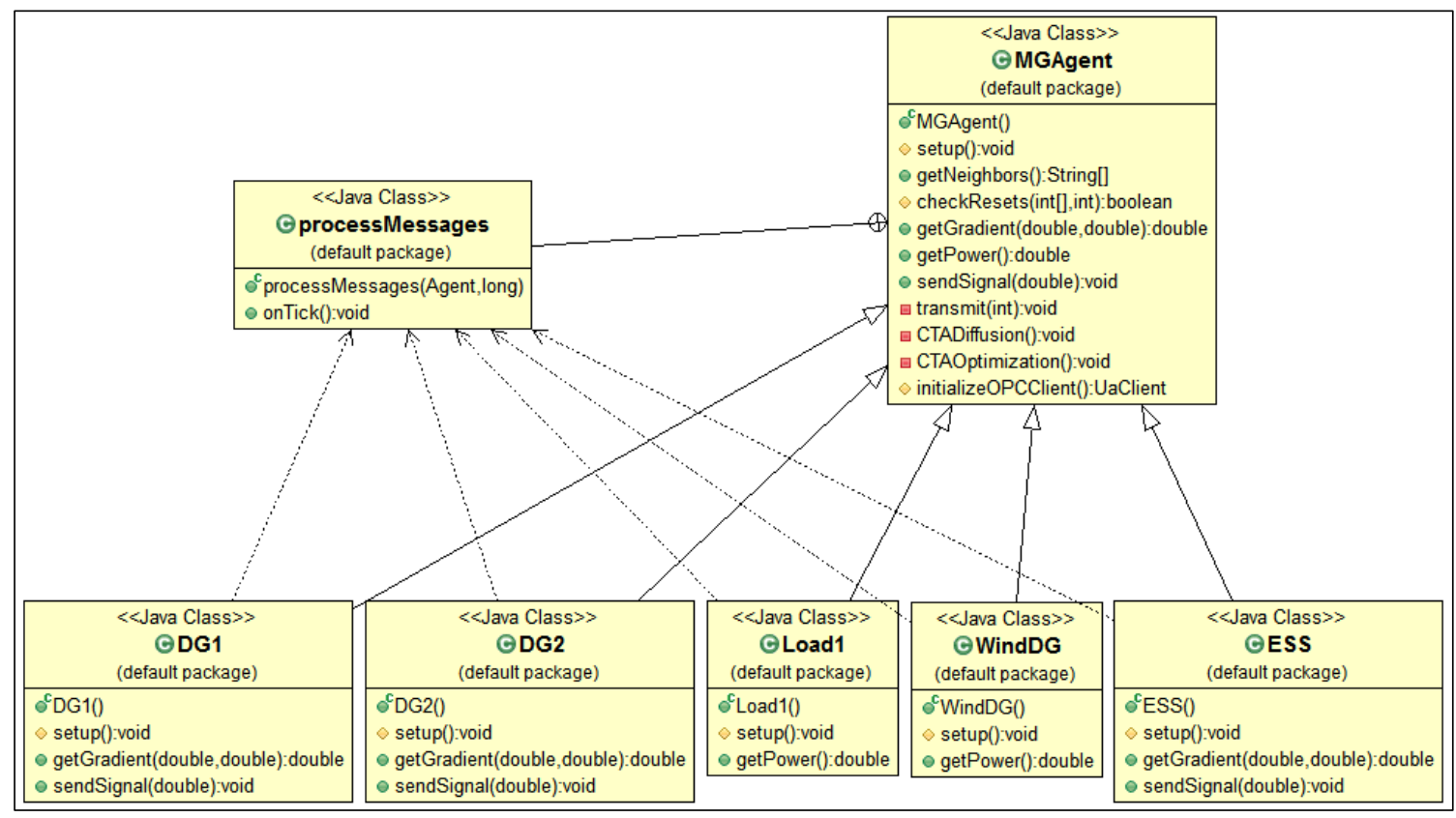

Figure 6.3.1: Multi-Agent System Java Implementation using JADE

The main class MGAgent extends the jade.core.Agent class that includes all basic agent functionality. JADE agents operate according to what are called behaviours that define how the agents act; the behaviours are added in the setup method of each agent. In this implementation, the MGAgent employs the TickerBehaviour, which repeats a process every so often; this was used to emulate and vary communication delays under the simulation platform described further along in the chapter. TickerBehaviour is extended by processMessages that calls the function onTick repeatedly after a predefined ticTime, which can be adjusted depending on the scenario. In turn, onTick processes the received messages 
from the other agents in the MG and calls CTADiffusion, which implements the IS diffusion algorithm.

As described in the previous section, after the first convergence of the IS diffusion, the net power in the MG is known and therefore the optimization procedure can be called; this is implemented in CTAOptimization. If the agent represents a DER, the getGradient method will return the gradient of that particular agent's cost function at the intermediate power point being optimized by diffusion. Additionally, DERs have the sendSignal function that updates the primary controller with the new power points. Conversely, the stochastic agents implement the getPower method, which reads the current power from the physical electrical connection. After each iteration of the diffusion updates (with or without optimization included), the transmit function is called to broadcast the new estimates to all neighbors.

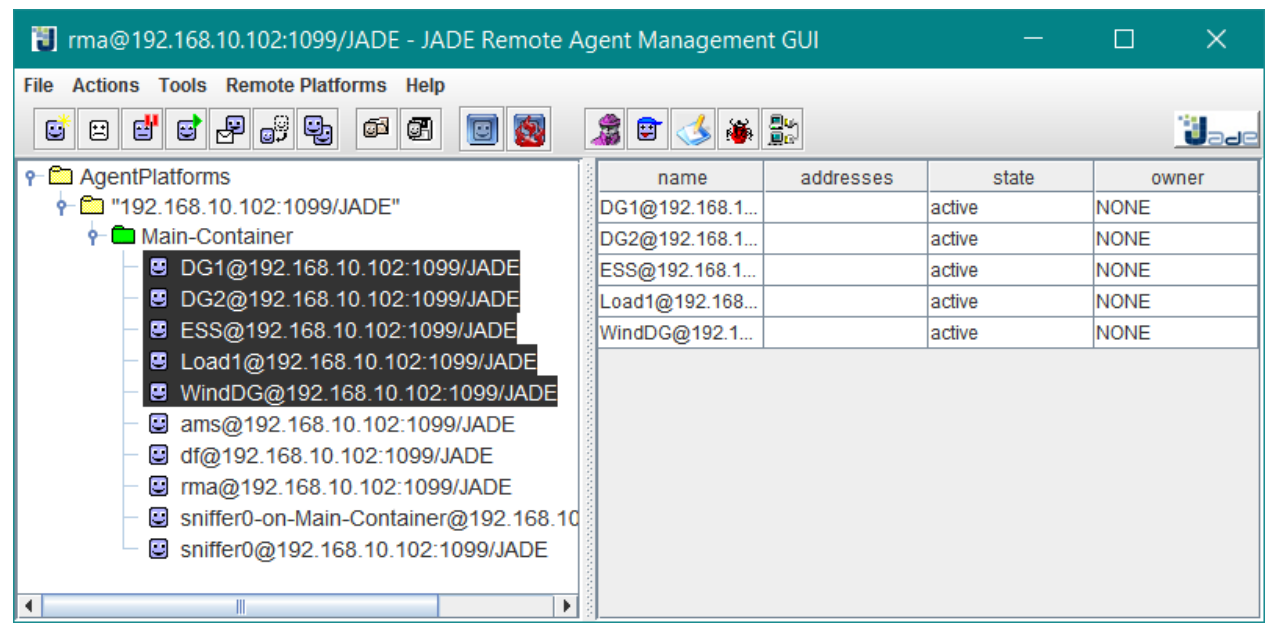

Figure 6.3.2: JADE Graphical User Interface

The JADE platform incudes a Graphical User Interface (GUI) that can be used to manage the agents and the communications. Although the MG agents are implemented using automated procedures, the GUI is helpful to visualize the system (Fig. 6.3.2.). 
Each agent represented in Fig. 6.3.1 can be seen instantiated in the JADE GUI; each agent has a unique name and physical address on the network. The GUI also provides a "sniffer" tool to see the inter-agent communications happening:

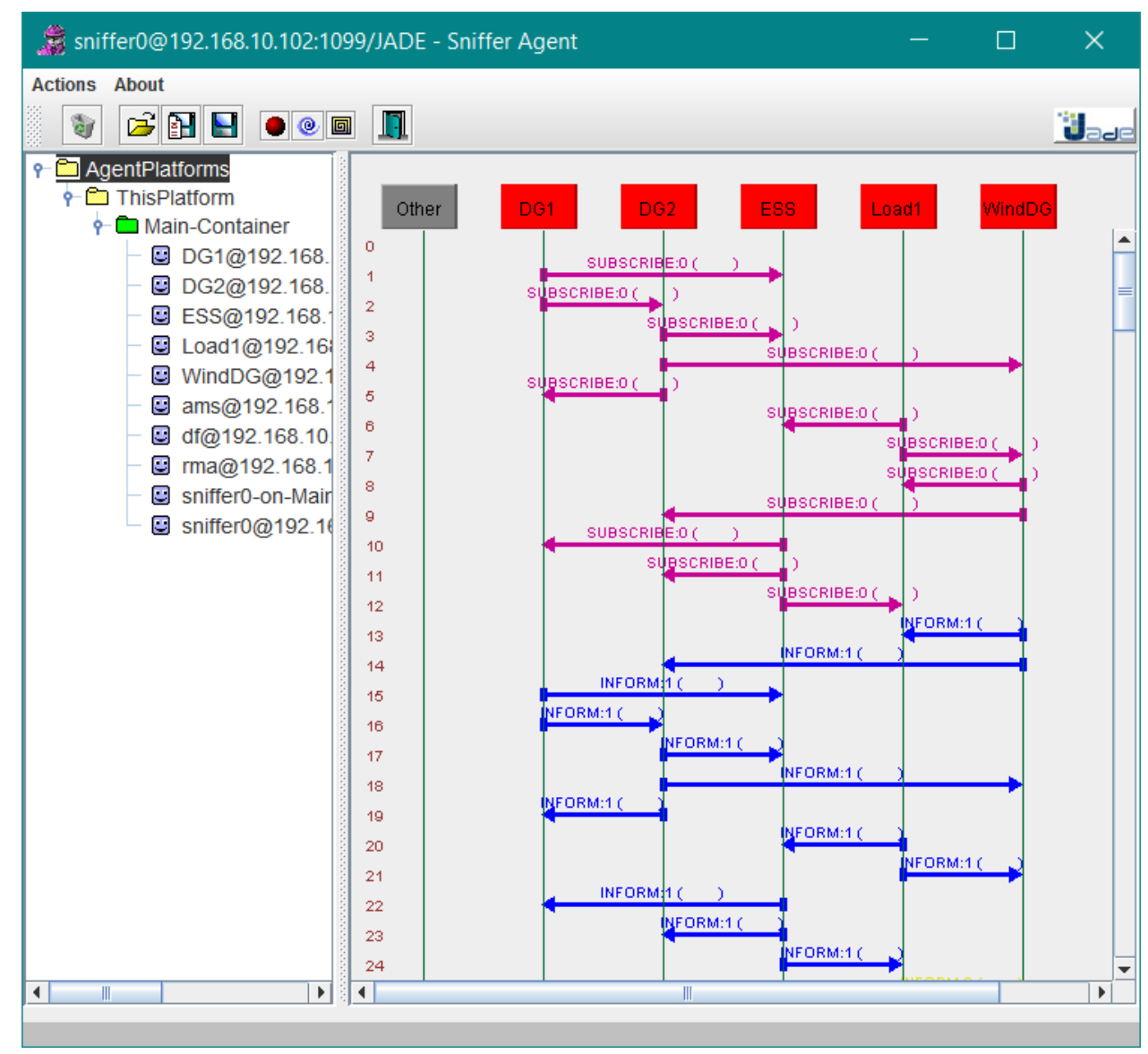

Figure 6.3.3: JADE Sniffer, Initial SUBSCRIBE and INFORM messages

At first, each agent sends an initial SUBSCRIBE message to its neighbors; in this case DG1 agent has DG2 and ESS as its neighbors. This will be further shown in the experimental setup section. The agents then start sharing their initial power readings (for stochastic agents) and the IS diffusion starts; these are the INFORM messages. In the sniffer, each iteration is marked by a different color, after a few iterations (18 in this case), the 
agents send a PROPOSE message, which indicates they have converged to the net power in the MG:

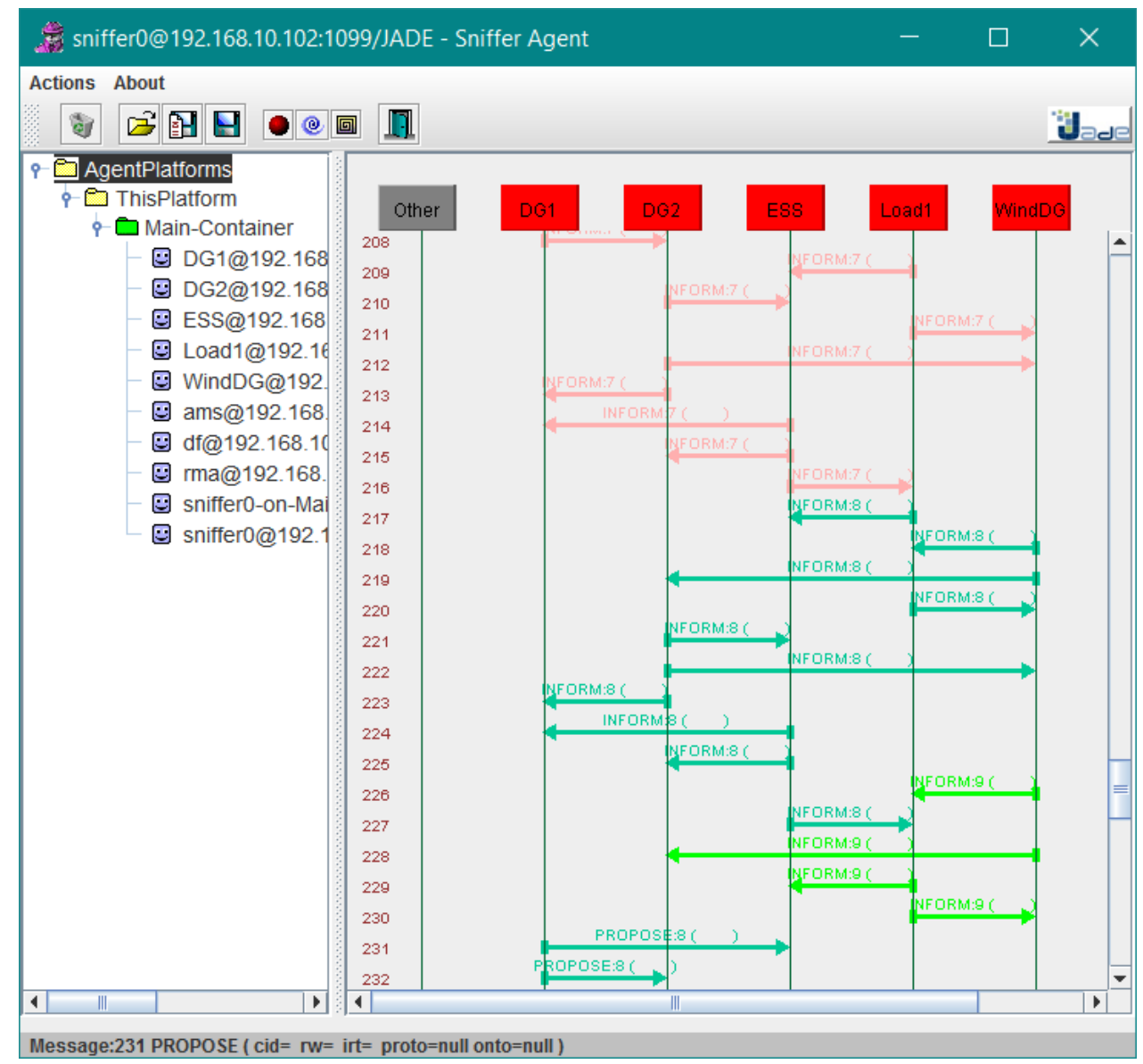

Figure 6.3.4: JADE Sniffer, PROPOSE message on IS convergence

As can be seen, DG1 reaches convergence on IS first, and sends the PROPOSE messages to the neighbors DG2 and ESS. The ACL messages have the following syntax:

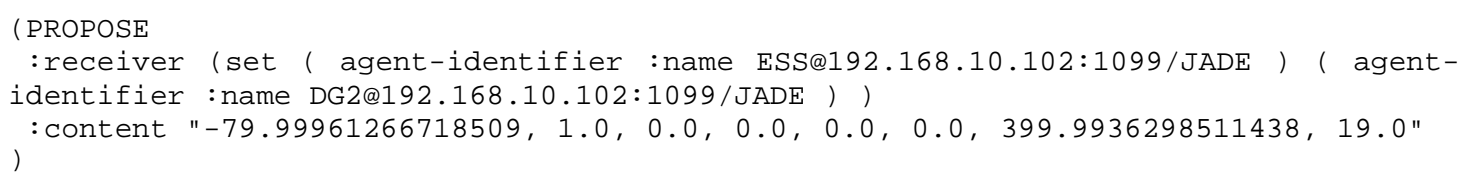

Therefore, the receivers are identified and the content of the message is the combined data packet, $p_{i}$, which includes the optimization, $w_{i}$, and power, $x_{i}$, estimates, as 
explained in Section 6.2. Also, an important detail to note on Fig. 6.3.4 is that the messages do not necessarily arrive in order, as can be seen on the INFORM messages being received by LOAD1 towards the end. Looking closely, the INFORM:9 from WindDG arrives before the INFORM:8 from ESS; therefore a queue needs to be implemented to process the messages from all neighbors in an orderly fashion to assure the convergence and accuracy of the diffusion communication.

The communication infrastructure is beyond the scope of this work; however, wireless peer-to-peer networks are proposed as an elegant solution in order to provide adaptability and ease of setup. JADE messages vary in size depending on how many neighbors an agent is communicating with and how many nodes exist in the MG network. The above message is only 224 bytes, but in general the ACL messages should be less than 1 kilobyte.

As an example, the $\mathrm{XBee-PRO}{ }^{\circledR}$ is considered as a possible communications infrastructure solution; it employs the ZigBee communication protocol to implement mesh and peer-to-peer physical networks at greater distances. The XBee-PRO® 900HP Module [38] has a bandwidth of $200 \mathrm{kbps}$, which would translate into more than 25 messages per second (200 kbps / 8 b/B / $1000 \mathrm{~B} / \mathrm{kB}=25 \mathrm{ps}$ ). This would mean IS convergence would most likely be reached in less than a second.

\subsection{SimULATION PLATFORM}

In order to prove the proposed control system before attempting to implement it in an experimental setup, the SimPowerSystems toolbox from Matlab was used to simulate a Microgrid (MG) and its electrical components. A hypothetical MG is devised that includes 
9 nodes: The Point of Common Coupling (PCC) with the utility grid, two Distributed Generators (DGs), one Battery Energy Storage System (BESS), one Commercial, one Critical and one Residential Load, one Photovoltaic (PV) farm and one Wind Turbine:

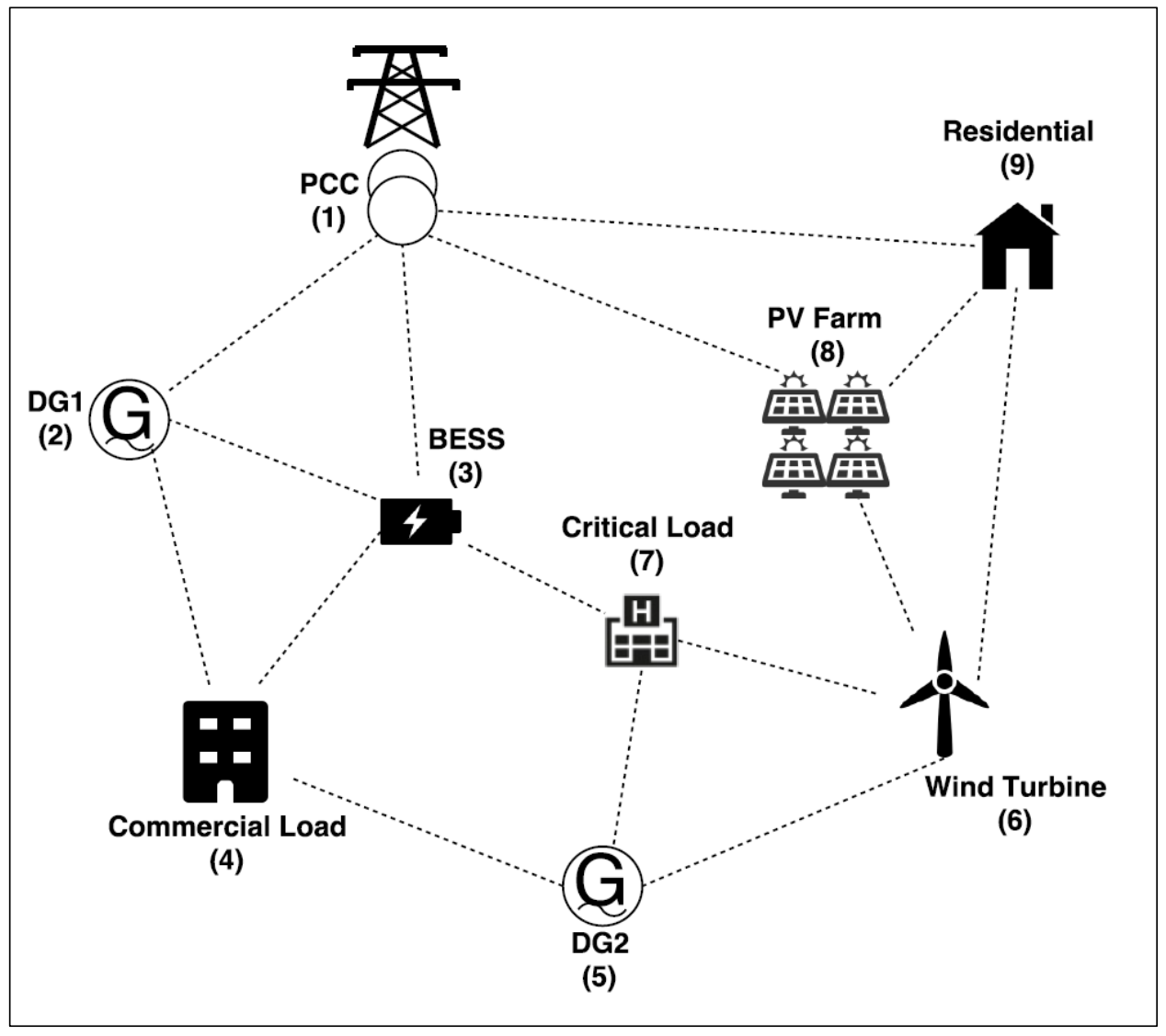

Figure 6.4.1: Hypothetical MG for Simulation

As can be seen in Fig. 6.4.1, each node is assigned a number and the communication links are denoted by the dotted lines. Therefore, a topology edge matrix, as described in Chapters four and five, can be constructed: 


$$
E=\left\{\begin{array}{lllllllll}
1 & 1 & 1 & 0 & 0 & 0 & 0 & 1 & 1 \\
1 & 1 & 1 & 1 & 0 & 0 & 0 & 0 & 0 \\
1 & 1 & 1 & 1 & 0 & 0 & 1 & 0 & 0 \\
0 & 1 & 1 & 1 & 1 & 0 & 0 & 0 & 0 \\
0 & 0 & 0 & 1 & 1 & 1 & 1 & 0 & 0 \\
0 & 0 & 0 & 0 & 1 & 1 & 1 & 1 & 1 \\
0 & 0 & 1 & 0 & 1 & 1 & 1 & 0 & 0 \\
1 & 0 & 0 & 0 & 0 & 1 & 0 & 1 & 1 \\
1 & 0 & 0 & 0 & 0 & 1 & 0 & 1 & 1
\end{array}\right\}
$$

Where $E$ is the network edge topology matrix. As an example, the first node, which is the PCC, connects with nodes 2, 3, 8 and 9 (including the self-connection). Therefore, by applying the metropolis rule described by Eq. 5.1.3, the following adjacency matrix is obtained:

$$
A=\left\{\begin{array}{rrrrrrrrr}
0.20 & 0.20 & 0.20 & 0 & 0 & 0 & 0 & 0.20 & 0.20 \\
0.20 & 0.35 & 0.20 & 0.25 & 0 & 0 & 0 & 0 & 0 \\
0.20 & 0.20 & 0.20 & 0.20 & 0 & 0 & 0.20 & 0 & 0 \\
0 & 0.25 & 0.20 & 0.30 & 0.25 & 0 & 0 & 0 & 0 \\
0 & 0 & 0 & 0.25 & 0.30 & 0.20 & 0.25 & 0 & 0 \\
0 & 0 & 0 & 0 & 0.20 & 0.20 & 0.20 & 0.20 & 0.20 \\
0 & 0 & 0.20 & 0 & 0.25 & 0.20 & 0.35 & 0 & 0 \\
0.20 & 0 & 0 & 0 & 0 & 0.20 & 0 & 0.35 & 0.25 \\
0.20 & 0 & 0 & 0 & 0 & 0.20 & 0 & 0.25 & 0.35
\end{array}\right\}
$$

As explained previously, these are the weights that the agents apply to the communication coming from the neighbors. The model of the three-phase MG is built in Simulink, where each node is a subsystem; the nodes are positioned to approximate Fig. 6.4.1's layout: 


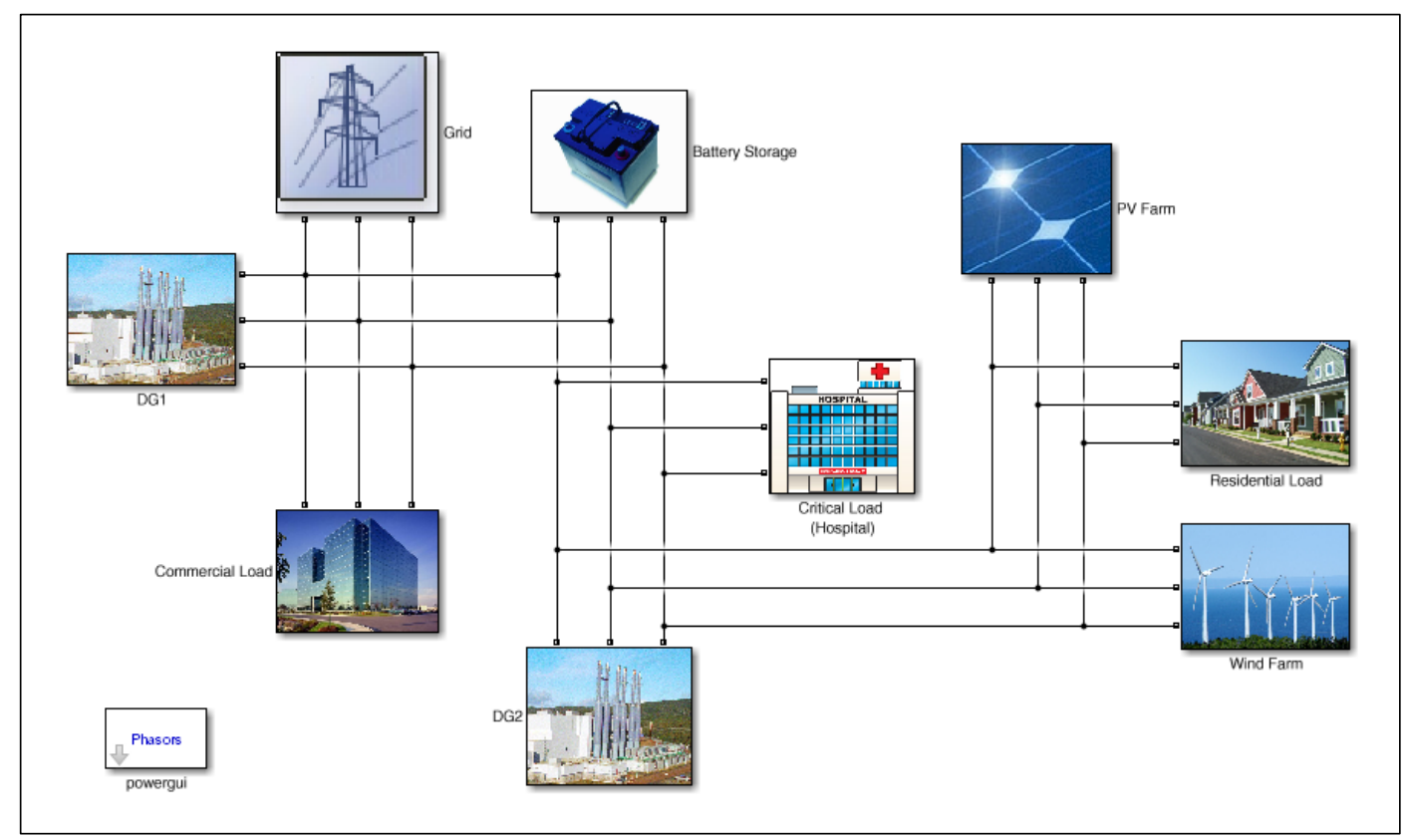

Figure 6.4.2: Simulink MG Model

Starting with the utility grid PCC, the subsystems are uncovered. As seen in Fig. 6.4.3, the grid is modeled by a three-phase source with a breaker controlled by the GRID_ON flag; this is the islanding PCC. The Matlab function block at the bottom of the diagram is the diffusion procedure, which takes the outputs of its four neighbors (plus its own output) as inputs, as well as the knowledge only known to the Grid Agent (GA): the grid power, grid price, and whether the MG is islanded. Additionally, there is a feedback of internal variables with a delay block, which acts as the memory of the agent that in Java 
would just be internal memory, but in Simulink has to be applied differently since the functions are called sequentially without storing variables.

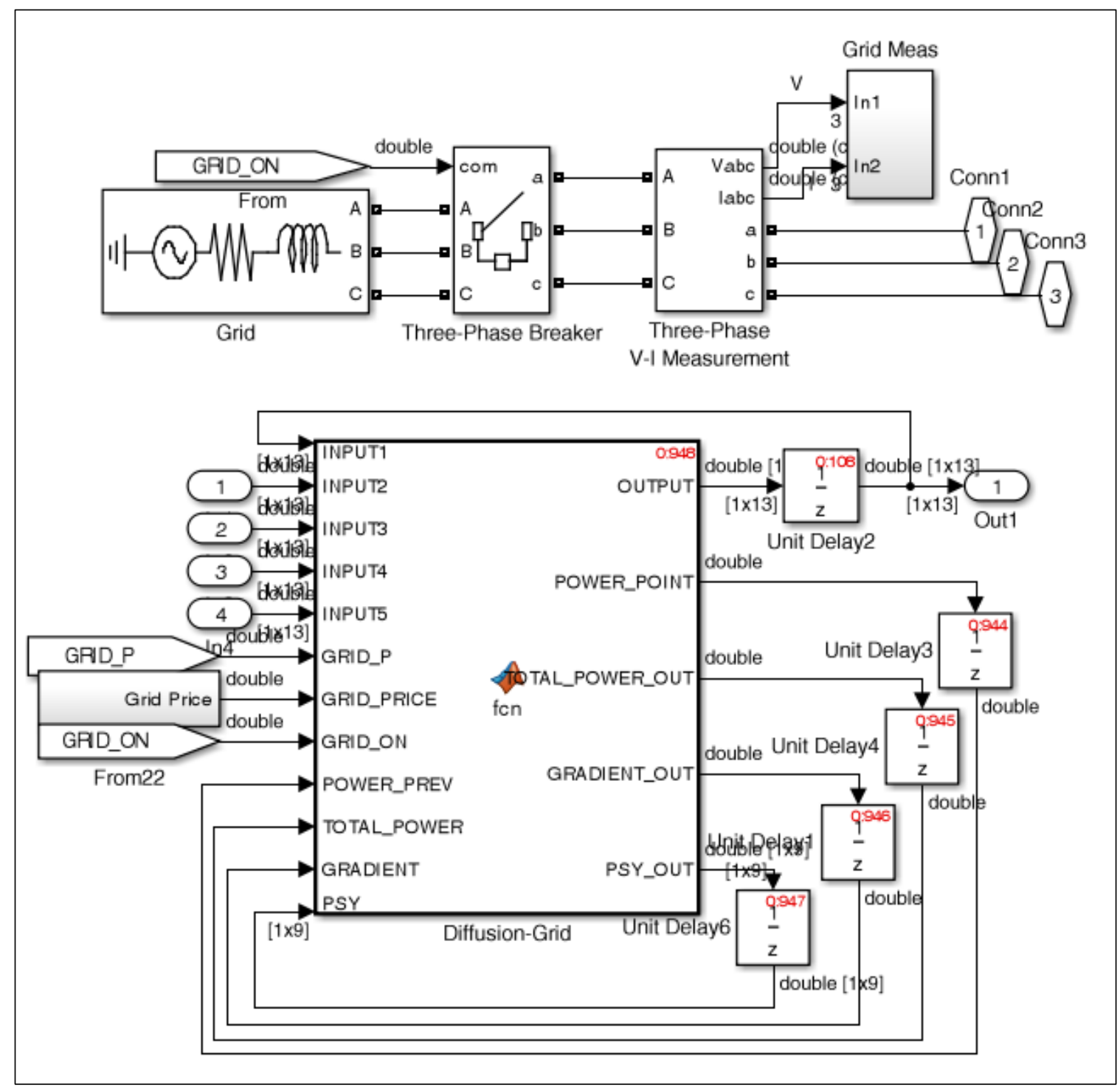

Figure 6.4.3: Simulink Grid Subsystem

The two DGs are modeled with a three-phase synchronous machine and they only differ on their rating; DG1 is rated at 2MW and DG2 at 1.5MW. The diffusion function is similar to the previous one except the diffusion optimization is used to update the No-Load Frequency $\left(f_{N L}\right)$ point of the generator's droop control (Fig. 6.4.4). This control is in another 


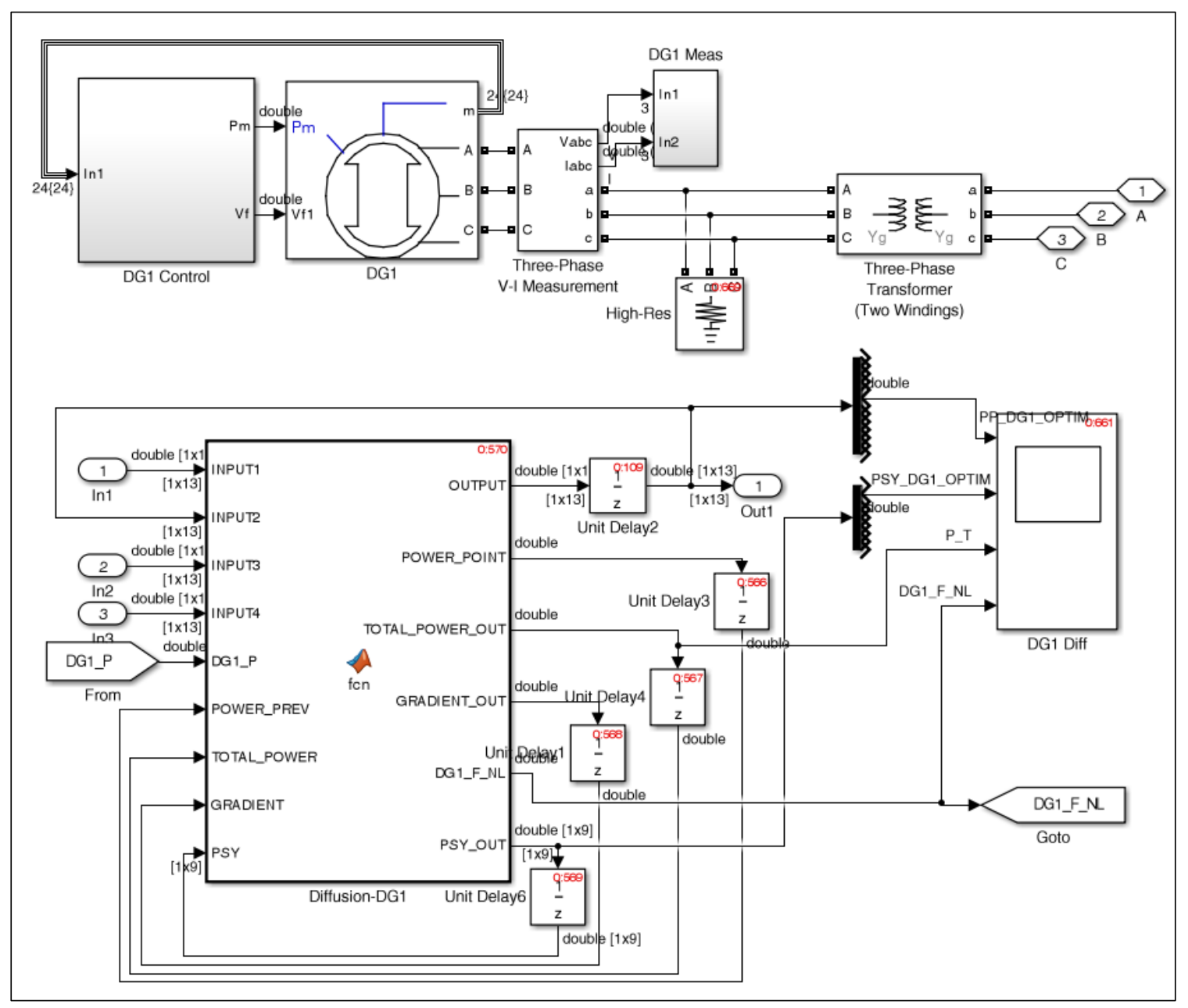

Figure 6.4.4: Simulink DG Subsystem

subsystem, which takes the measurements from the generator and the $f_{N L}$ from the diffusion with a Proportional Integral (PI) controller and a feedback equal to the droop slope (Fig. 6.4.5).

The $R$ feedback corresponding to the droop slope is calculated for a $1 \mathrm{~Hz}$ tolerance band (59.5-60.5 Hz) in per unit using Eq. 3.3.2, the negative sign being implemented in the addition block:

$$
R=59.5 / 60-60.5 / 60=-1.67 \%
$$




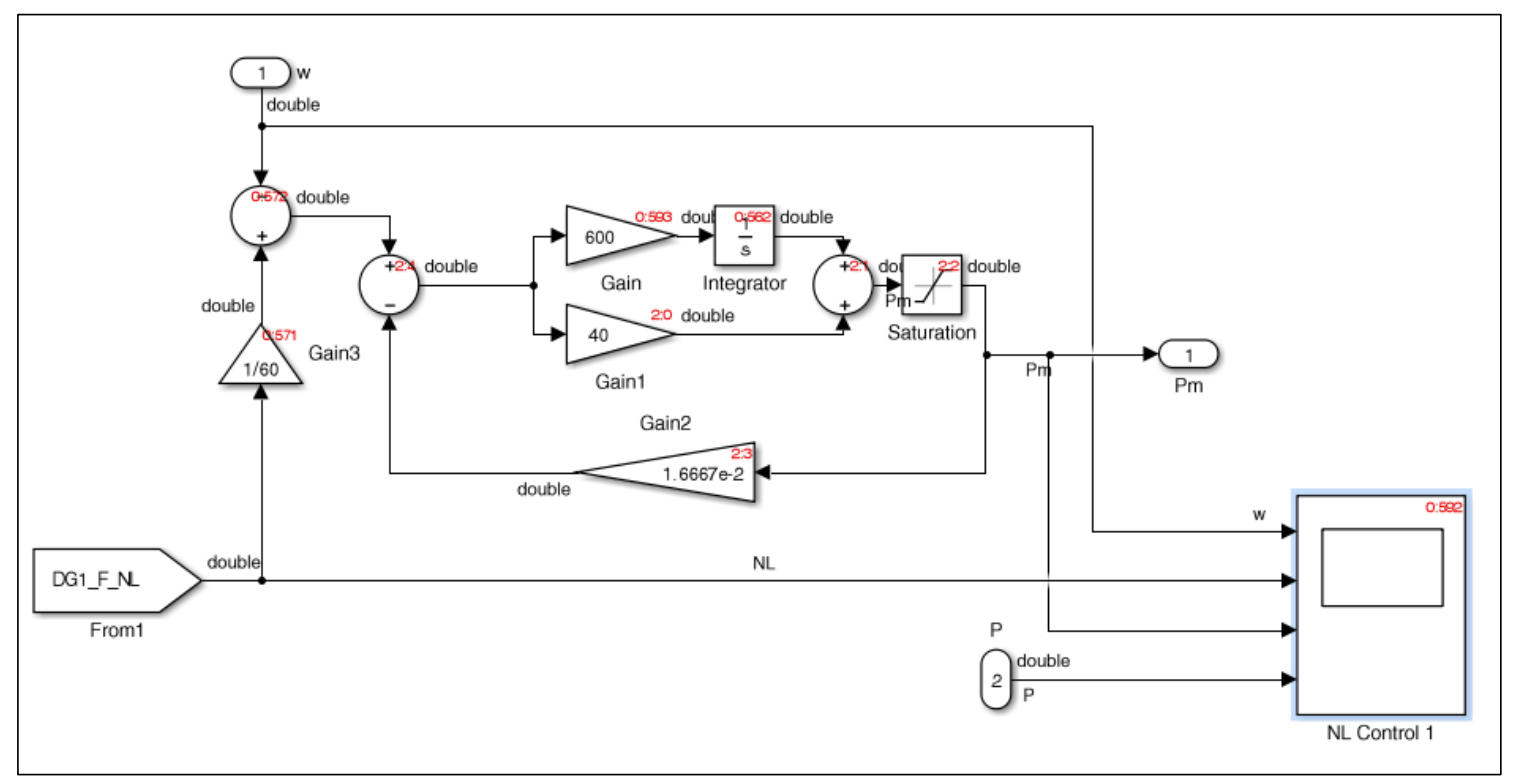

Figure 6.4.5: Simulink DG Droop Control

The voltage regulator and excitation of the DGs is left to the IEEE type 1 synchronous machine voltage regulator, which is a standard block included with the SimPowerSystems toolbox. The BESS is modeled as a three-phase current injector that is adjusted by a droop control equivalent to the DGs; in this way all DERs share the power imbalances according to their ratings. As seen in Fig. 6.4.6 the diffusion function is the same as the DGs.

The three-phase current injector is controlled by current references (Ia_BATT, $\left.I b \_B A T T\right)$, which are adjusted according to the output power reference from the droop control and the voltage readings (Vab_BATT, Vbc_BATT). Additionally, it can be seen at the bottom of the diagram the blocks that update the State of Charge (SOC) of the battery, which take into account the battery efficiency (Fig. 6.4.6). 


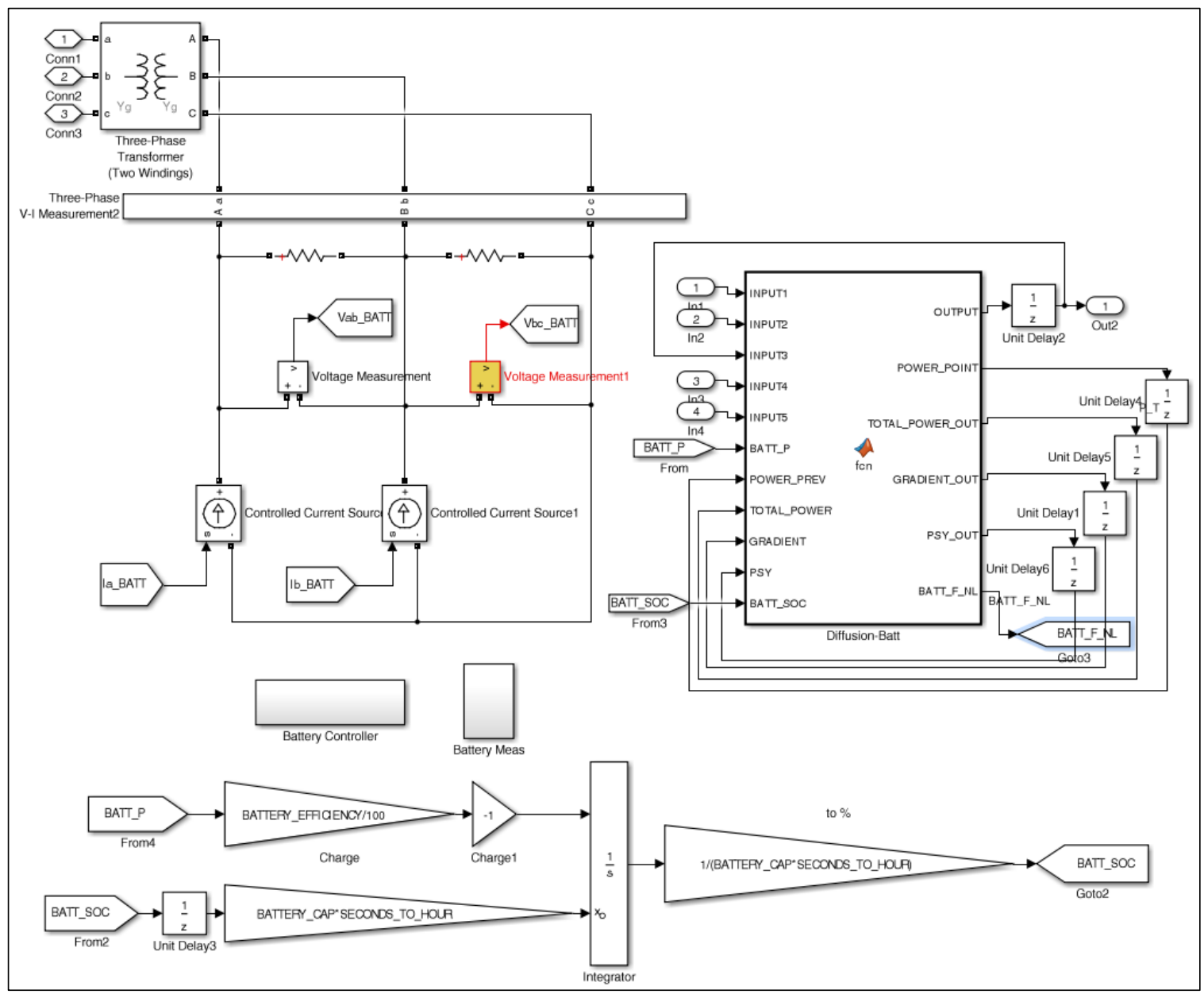

Figure 6.4.6: Simulink BESS Subsystem

Loads and RES (stochastic) nodes are also modeled as current injectors; however, the output (or input) of these is programmed into the model as a load or generation profile, which will be explained shortly. For IS diffusion, these agents have, as an input, the current power being drawn from, or fed into, the MG (Fig. 6.4.7). 


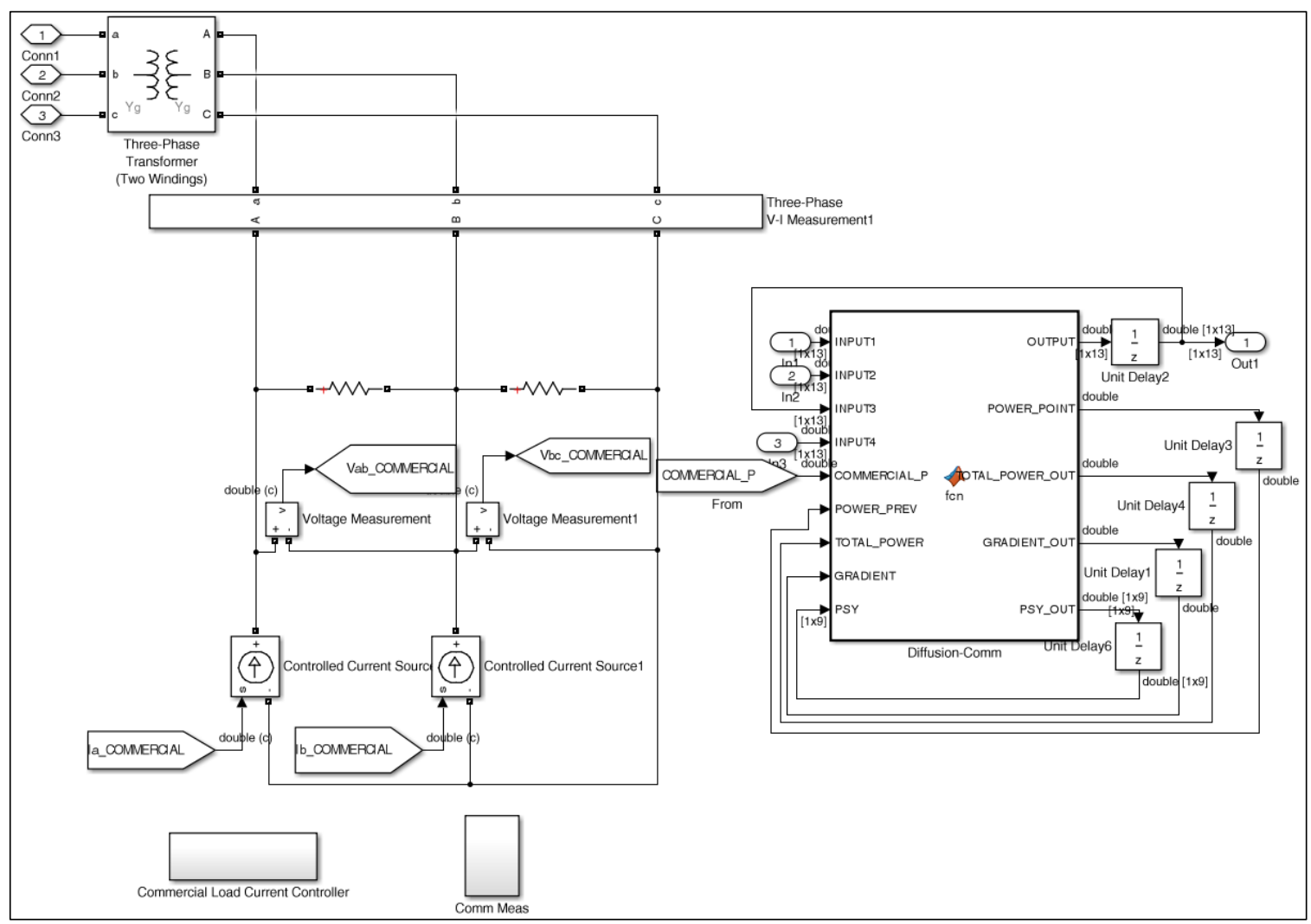

Figure 6.4.7: Simulink Stochastic Subsystem

There are three types of stochastic load profiles and two types of renewable generation profiles. The commercial load profile is very intensive during business hours and drops off during night time; it has a peak of 1.5 MW (Fig. 6.4.8).

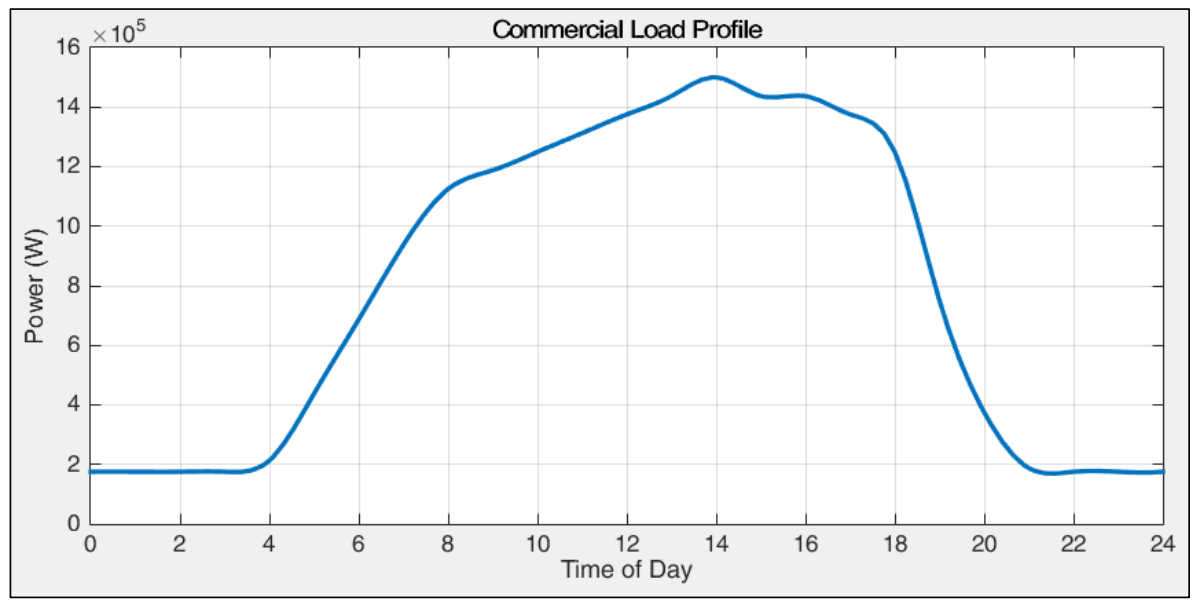

Figure 6.4.8: Commercial Load Profile 
The critical load profile has a higher off-peak base and a maximum load of 1.2 MW:

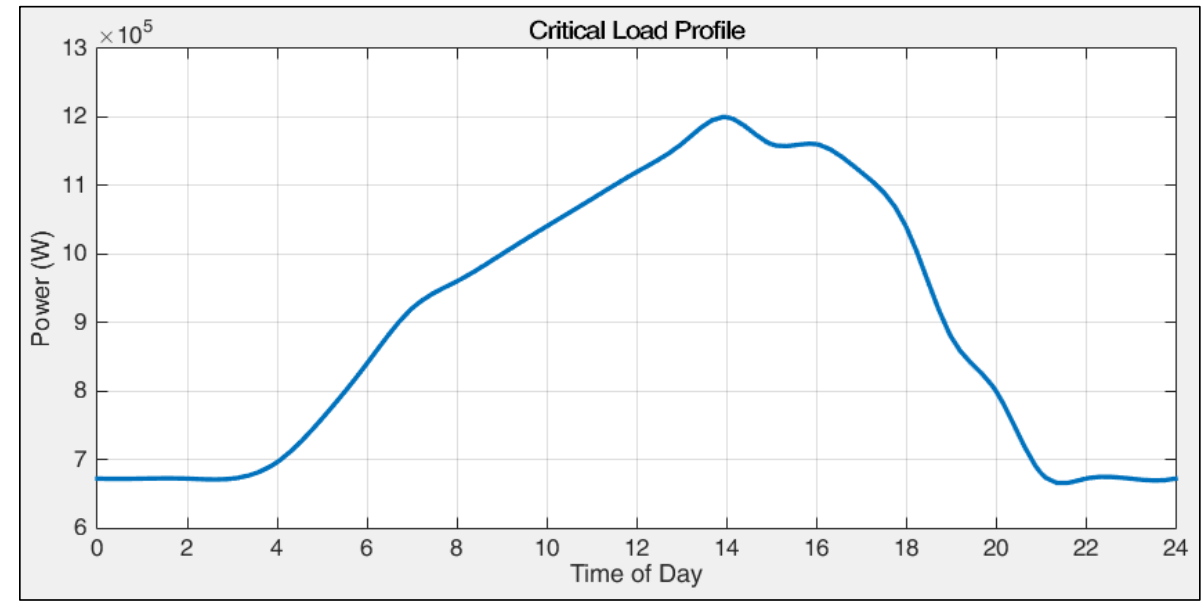

Figure 6.4.9: Critical Load Profile

The residential load has a maximum of $500 \mathrm{~kW}$ and has a morning “mini-peak” and a large evening peak, with the minimum during the early hours of the morning:

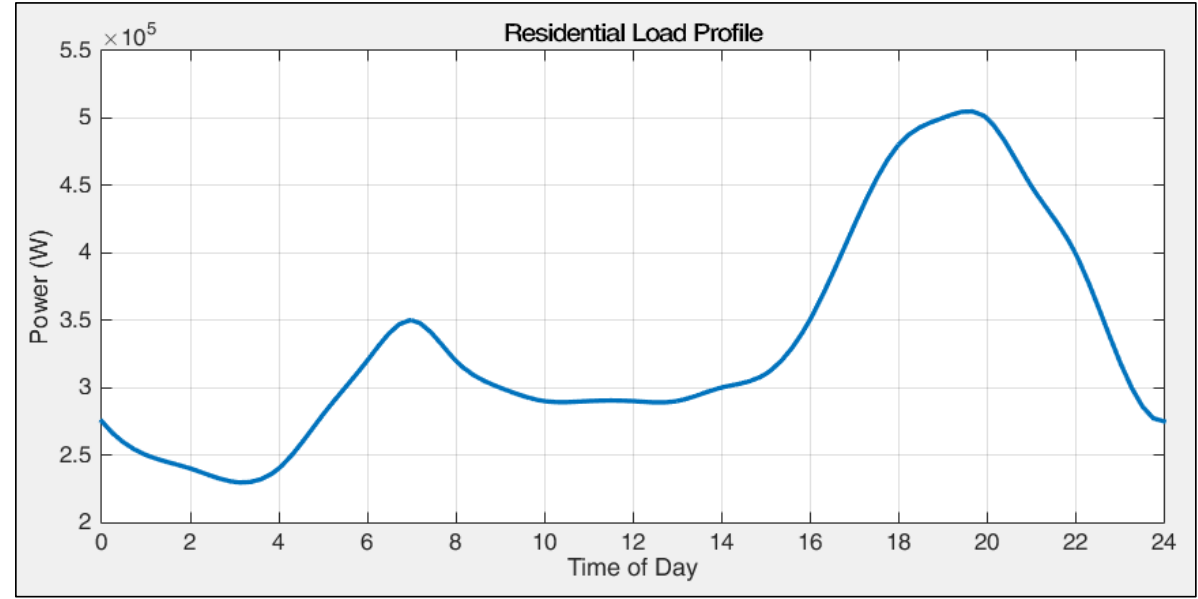

Figure 6.4.10: Residential Load Profile

The wind and solar profiles were taken from actual meteorological data from NREL's Solar Prospector [39] for the city of Key Biscayne, FL, which is an island and therefore a good case study for a MG. The wind data provided is at an elevation of $10 \mathrm{~m}$ 
and thus has to be adjusted for the height of a wind turbine. The Inerjy Ecovert 250 [40] with a height of $60 \mathrm{~m}$ was taken as a suitable candidate for the wind conditions present at this location. The wind is adjusted with the following formula [22]:

$$
\left(\frac{v}{v_{0}}\right)=\left(\frac{H}{H_{0}}\right)^{\alpha}
$$

Where $v$ is the adjusted wind speed, $v_{0}$ is the original wind speed, $H$ is the adjusted height, $H_{0}$ is the original height, and $\alpha$ is the friction coefficient, which varies according to terrain characteristics; $\alpha=0.2$ is used, which corresponds to high scrubs, hedges and shrubs. The adjusted wind speed is then mapped to the output power curve of the turbine:

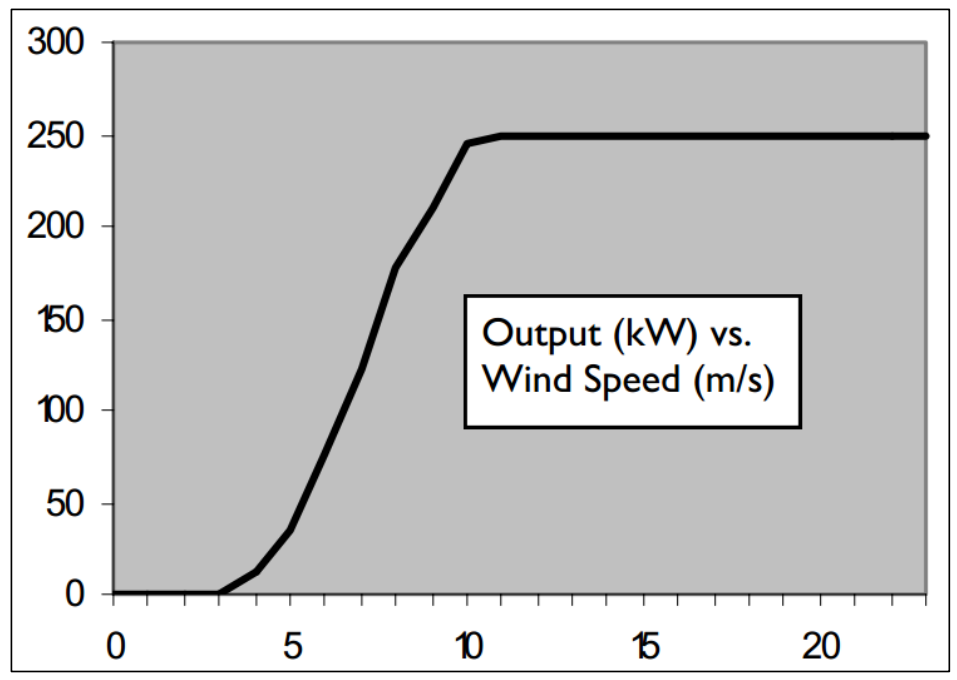

Figure 6.4.11: Inerjy EcoVert 250 Output Power vs Windspeed [40]

Taking a day that exhibited variable wind conditions (3/20/2009), the following power vs time of day profile was obtained (Fig. 6.4.12). A maximum of 1MW was used in the simulation, which would correspond to 4 of these turbines in a wind farm. 


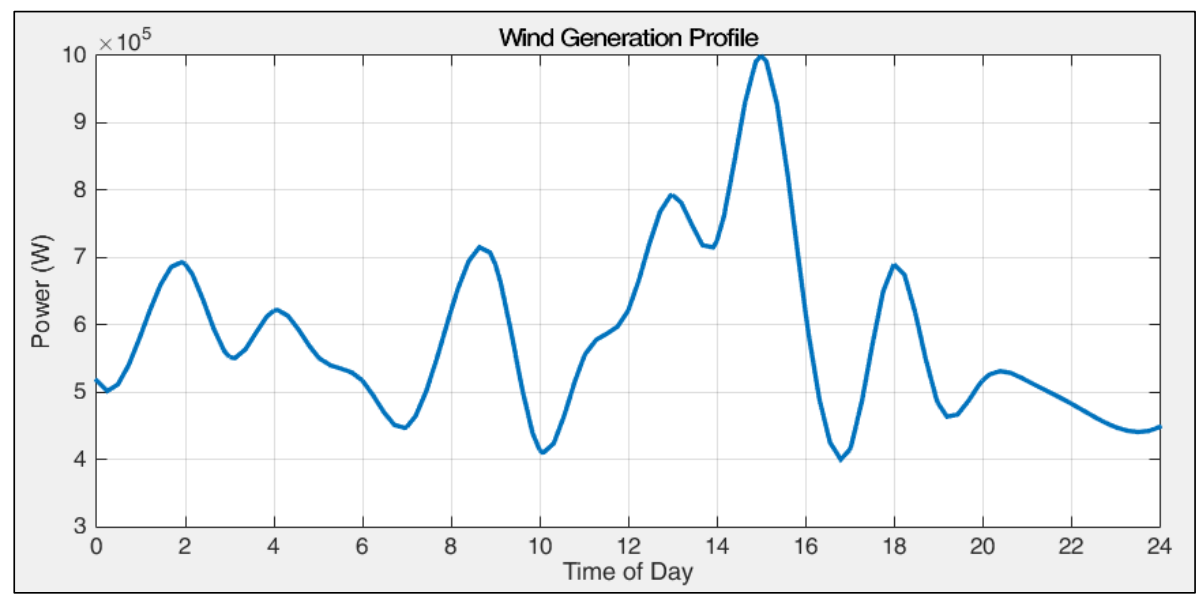

Figure 6.4.12: Wind Turbine Generation Profile for the city of Key Biscayne (3/20/2009 @ 60m)

The solar profile was taken for the same day, adjusted to a $1 \mathrm{MW}$ solar PV farm:

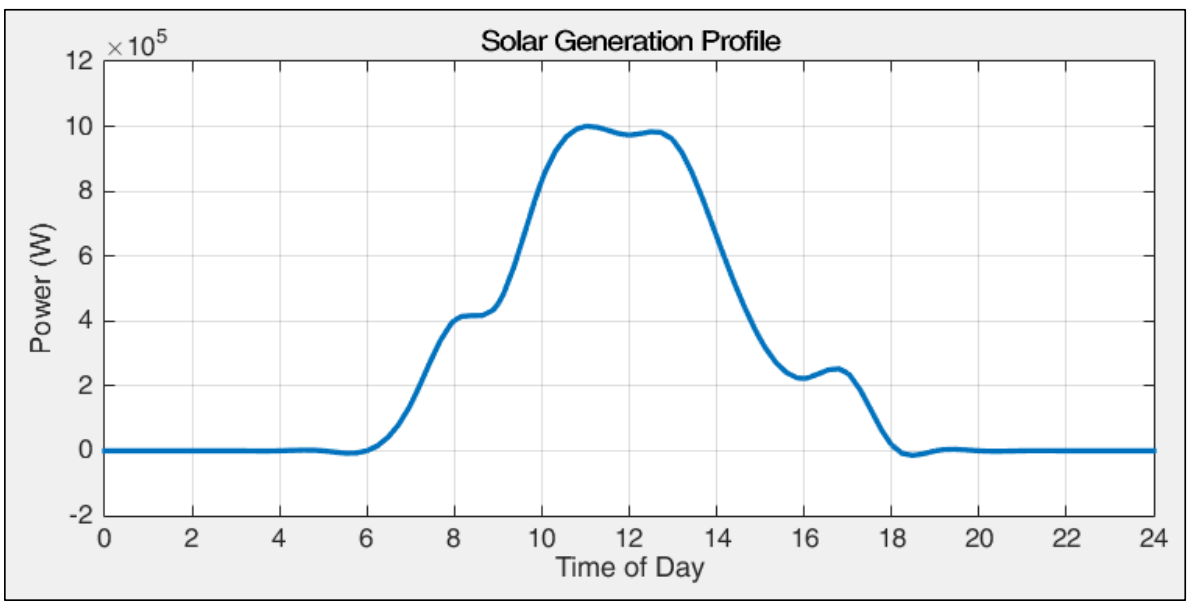

Figure 6.4.13: Solar PV Generation Profile for the city of Key Biscayne (3/20/2009)

\subsection{SIMULATION RESULTS}

The simulation was run for 48 seconds in Simulink, where 2 seconds of simulation represent an hour of the day. The Battery Energy Storage System (BESS) begins with 50\% State of Charge (SOC) and 95\% efficiency each way (charging and discharging). 
Information Sharing (IS) diffusion is accurate to six significant figures, and the diffusion functions were run every $5 \mathrm{e}-4 \mathrm{~s}$, which translates to about one a second in real time.

\subsubsection{ISLANDED MICROGRID}

First, the islanded Microgrid (MG) under the load and Renewable Energy Source (RES) profiles described in the previous section is analyzed with the two DGs' cost functions, as described in Section 5.4 (Eq. 5.4.8-5.4.13), and the BESS cost function following the formulation in Section 4.3:

$$
\begin{gathered}
F_{B}=20+8 e-5\left(P_{B}+3 P_{B, \max }(1-S O C)\right)+8 e-11\left(P_{B}+3 P_{B, \max }(1-S O C)\right)^{2} \\
\frac{d F_{B}}{d P_{B}}=8 e-5+8 e-11\left(2 P_{B}-6 P_{B, \max }(S O C-1)\right) \\
-500 k W \leq P_{B} \leq 500 k W
\end{gathered}
$$

The BESS has a rating of $500 \mathrm{~kW}$ (charging and discharging) and storage capacity of 500kWh. First, the IS diffusion is evaluated and compared to the actual values. Here, the MG net power $P_{N}$ is being diffused through the network:

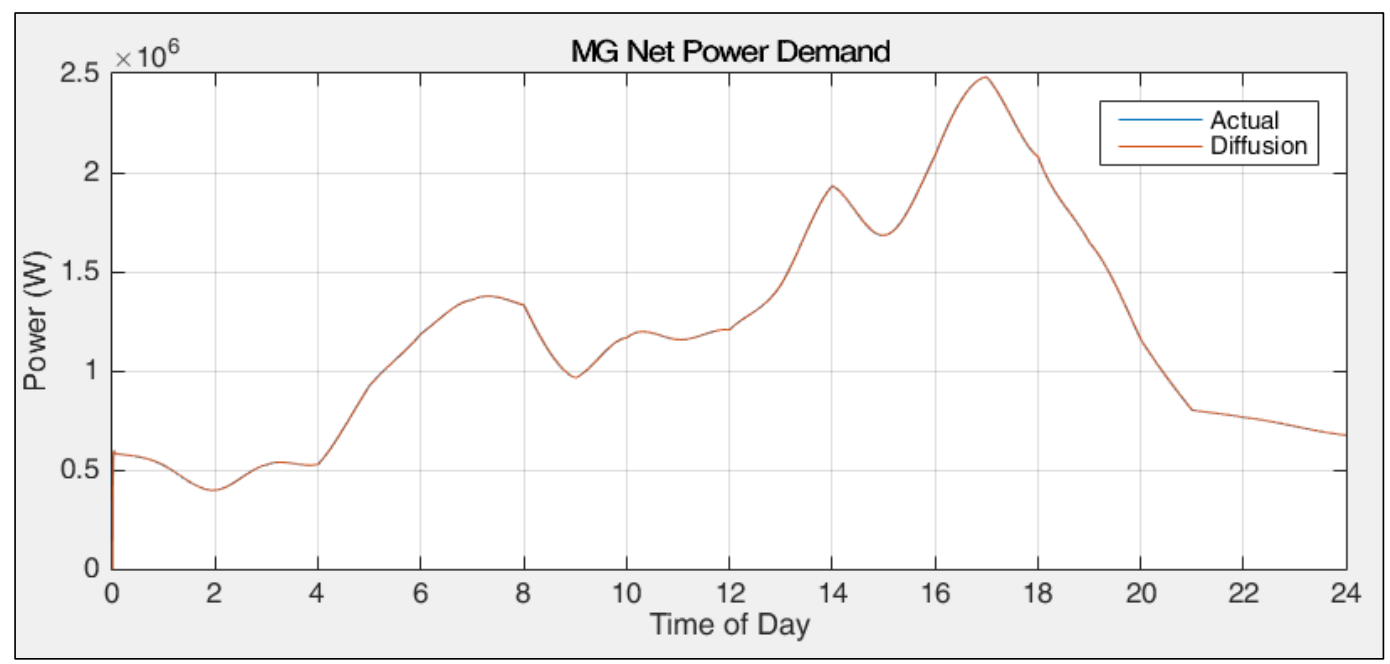

Figure 6.5.1: MG Net Power Demand, Actual vs Diffusion 
The net power profile is similar to the residential load profile; this is because the solar and wind power output around noon are large and compensate for the high commercial and critical loads. From the view in Fig. 6.5.1 the difference between the actual value and the diffusion convergence can't be distinguished, meaning the IS diffusion is closely tracking the net power of the MG. Looking more closely, the slight differences can be noticed:

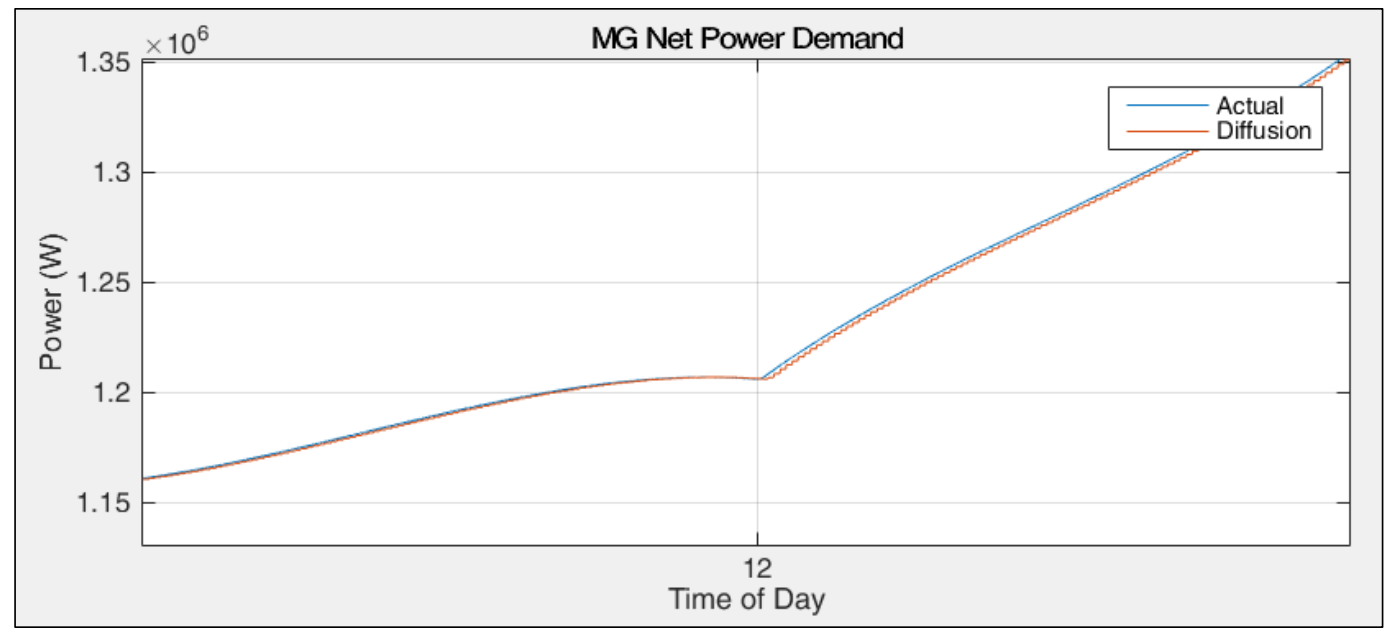

Figure 6.5.2: MG Net Power Demand, Actual vs Diffusion, Close-up

In Fig. 6.5.2, it is apparent how diffusion is a step behind the actual power, but the difference is negligible. Next, the output of the Distributed Energy Resources (DERs) is compared to the net power demand (Fig. 6.5.3).

For this islanded scenario, the output of the three DERs add up to the net power demand. It can be seen how, in the beginning, the BESS has a large negative (charging) output because it was at only 50\% SOC and the load was relatively low. The DGs pretty much track the net power demand according to the ED point, which will be compared to a centralized controller in the following figure (Fig. 6.5.4). 


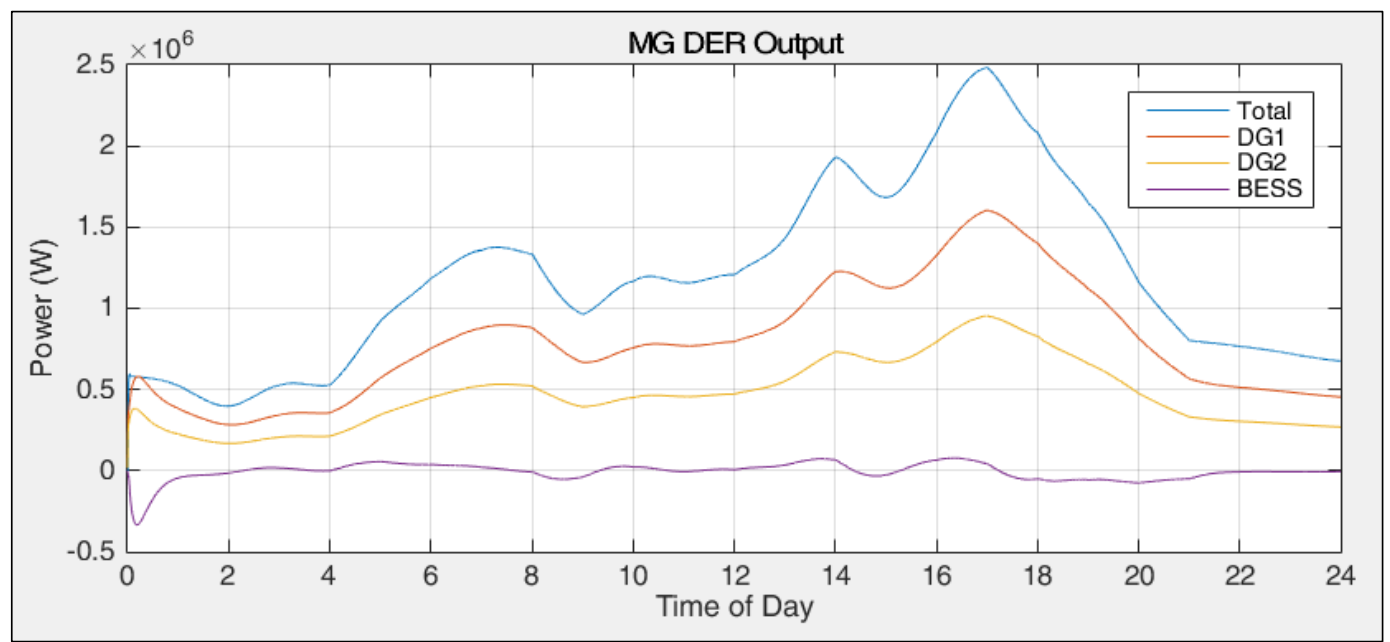

Figure 6.5.3: MG DER Output, Islanded

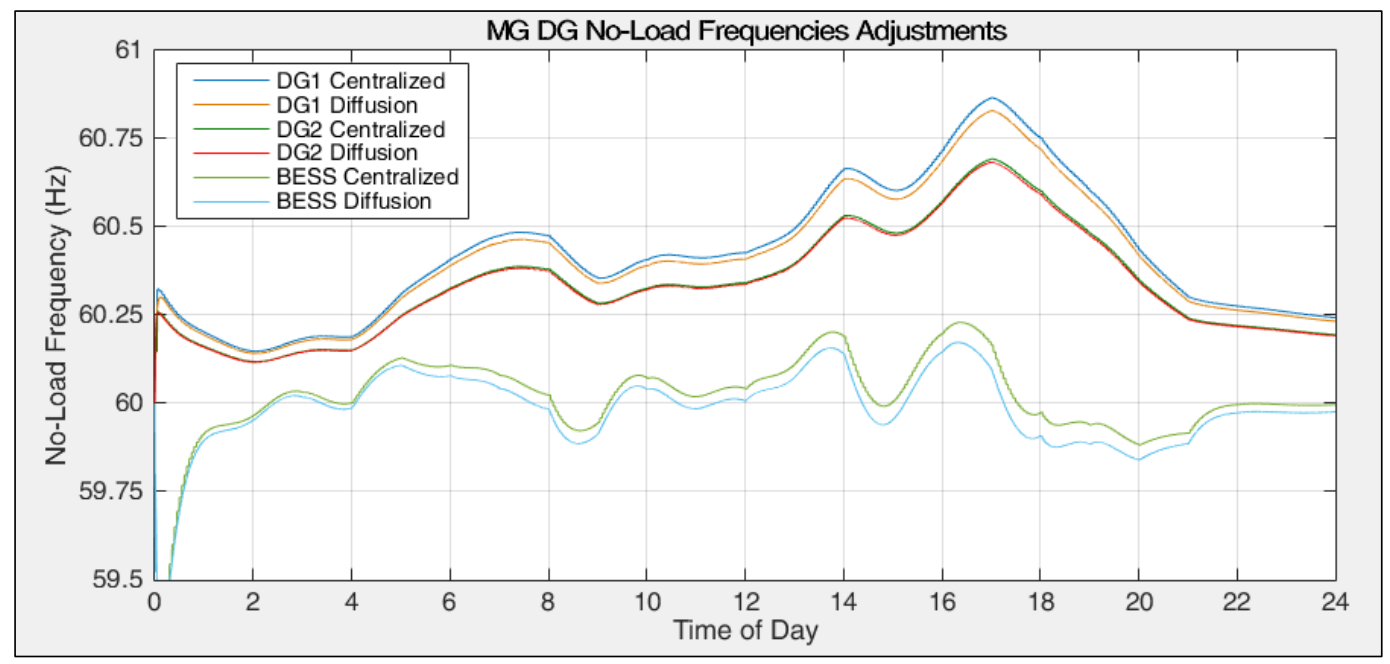

Figure 6.5.4: DER No-Load Frequency Adjustments, Islanded

Here, the optimized power points of the three DERs adjust the No-Load Frequencies $\left(f_{N L}\right)$ of their respective assets according to the relationship described by Eq. 6.2.1. For the BESS, a value of $f_{N L}$ below 60 means it is being set to charge. It is shown how they closely follow what the centralized solution would determine. Taking a closer look, the performance is better compared (Fig. 6.5.5). 


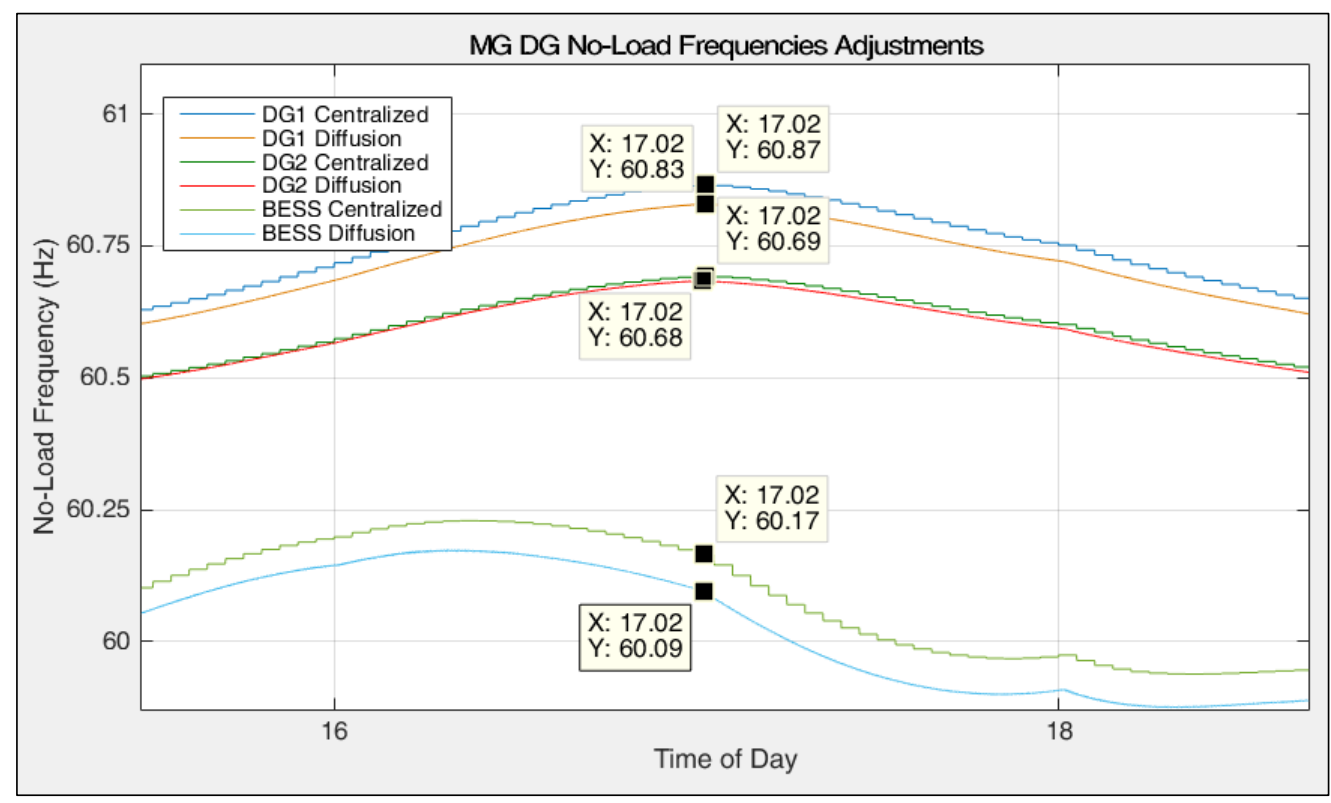

Figure 6.5.5: DG No-Load Frequency Adjustments, Islanded, Close-up

Again, the tradeoff is observed between accuracy and adaptability, but the solution is $99.9 \%$ accurate, which is a small price to pay for the flexibility offered by the platform. The behavior of the BESS can be observed by looking at the net demand, output power and SOC of the battery (Fig. 6.5.6).

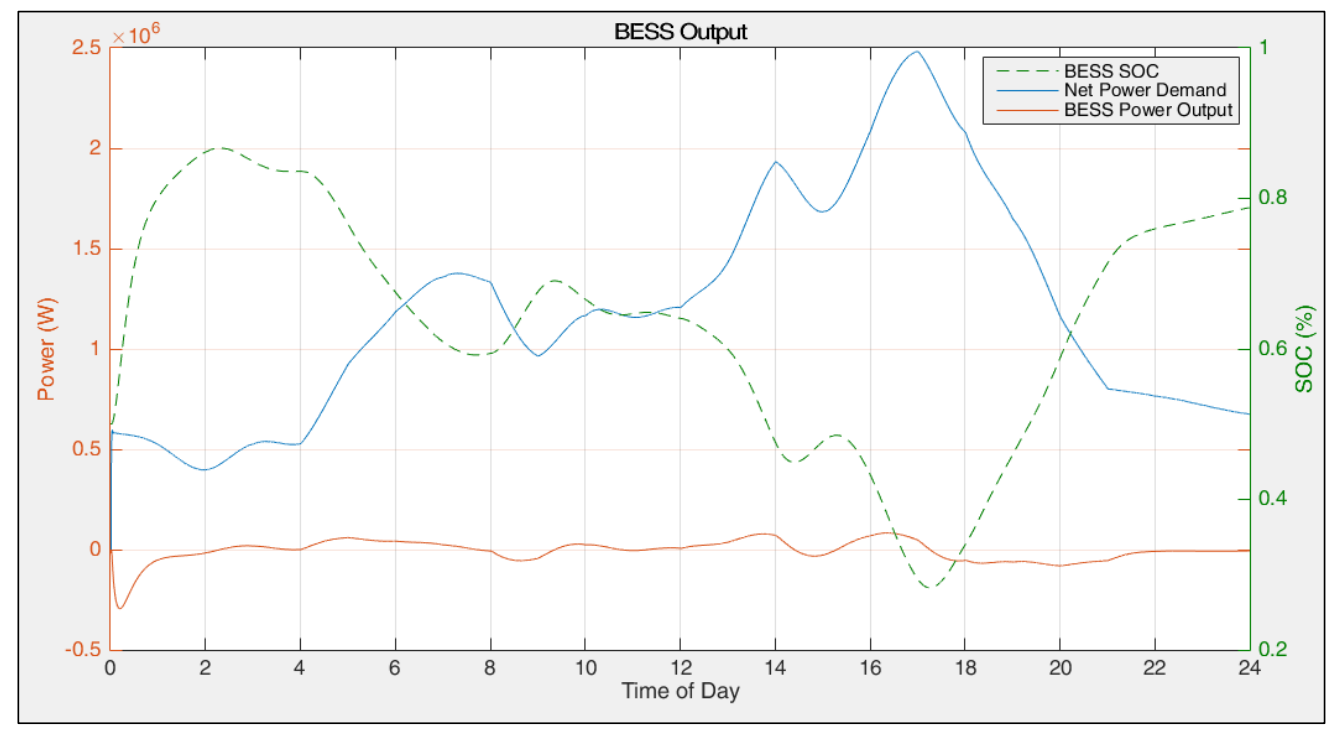

Figure 6.5.6: BESS Power Output vs SOC, Islanded 
It can be seen how SOC is inversely correlated to the net power demand in the MG. This makes sense because as the demand of the MG rises, the DERs are forced to operate at higher marginal costs, and thus the battery is driven to discharge more of its energy, as described in Section 4.3.

The MAS control was designed to maintain the stability of the MG and adjust the frequency when islanded. The following figure shows the frequency and voltage throughout the simulation (Fig. 6.5.7).

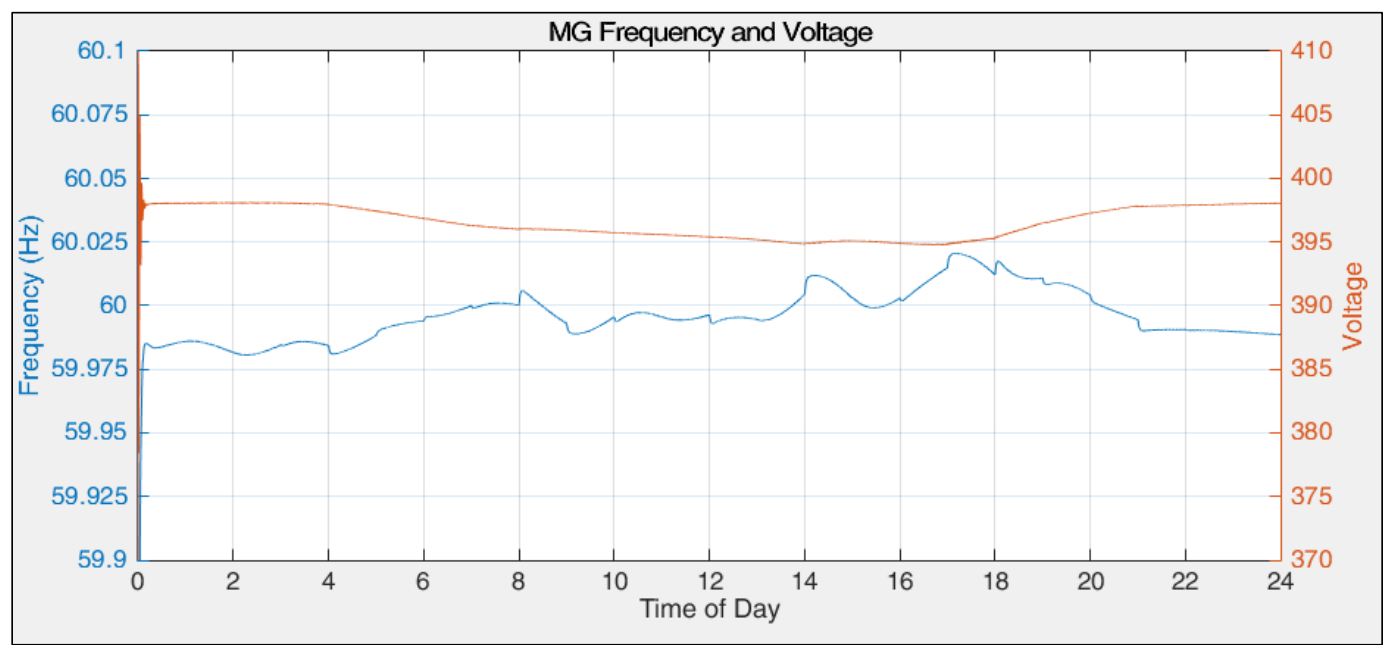

Figure 6.5.7: MG Frequency and Voltage, Islanded

As can be seen, the frequency is maintained within a very narrow band of nominal frequency; the deviations are maintained within $0.025 \mathrm{~Hz}$, which is less than $0.05 \%$ divergence. The voltage is also maintained within acceptable limits at over 395V for a 400V nominal (1.25\% deviation).

\subsubsection{GRID CONNECTED MG}

The second scenario is a repeat of the first, but now the MG is connected to the utility grid. There is a simple Time of Use (TOU) rate structure, which, as the term suggests, 
varies the electric rates according to the time of day. This is done to economically incentivize customers to consume electricity during periods of low demand and reduce their consumption during peak times to alleviate stress on the system:

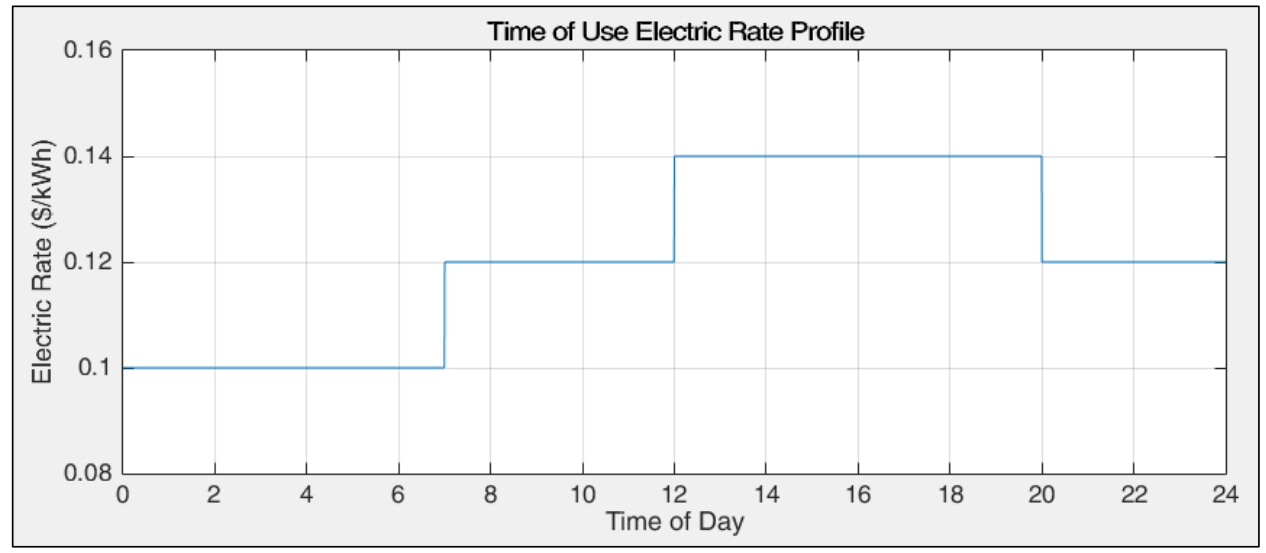

Figure 6.5.8: Electric Utility TOU Rate Structure

As can be seen, the rate structure roughly follows the demand profiles. The IS diffusion again closely tracks the net power demand, as shown in Fig. 6.5.1 and 6.5.2, however, the DER outputs follow completely different profiles (Fig. 6.5.9).

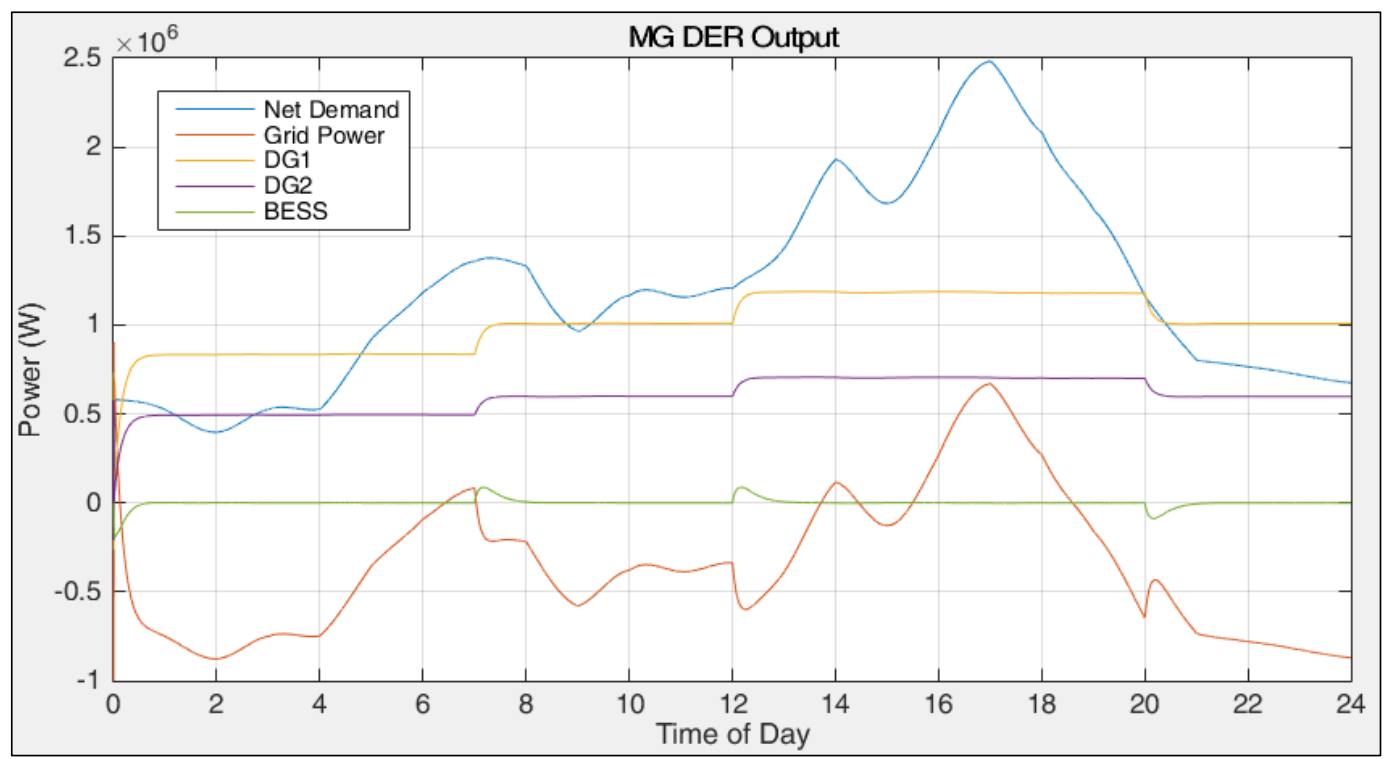

Figure 6.5.9: MG DER Output, Grid-Connected 
The MG exports power to the utility grid for most of the day, except at peak hours, when it is more economical to import some power than to produce more locally. The two DGs quickly settle at their Economic Dispatch (ED) point after each change in the TOU rate. As explained in Section 4.3, the marginal cost of all DERs for a grid-connected MG settle at the electric rate, which is essentially a constant marginal cost. The grid power then fills or withdraws whatever deficit is left from the DERs to meet the net power demand. The no-load frequency adjustments in this scenario are much more precise due to the long periods of time with constant marginal costs:

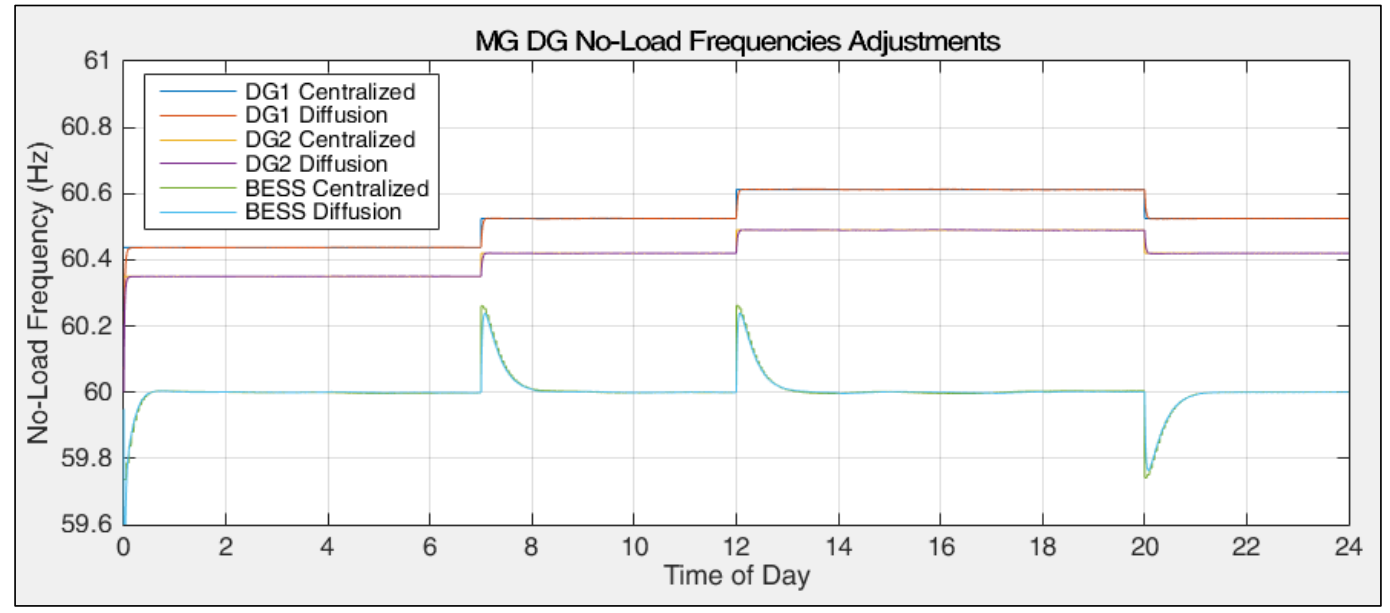

Figure 6.5.10: DER No-Load Frequency Adjustments, Grid-Connected

The tracking is very precise, but again the transient performance is observed when zoomed in Fig. 6.5.11. As shown, the tracking is very accurate, and the ED is reached in 0.1 , which is equivalent to 3 minutes in real-time with only one diffusion iteration per real-time second. With the previously mentioned real-world estimate of 25 iterations per second, this process would only take 7 seconds to settle. The BESS in turn is driven to 


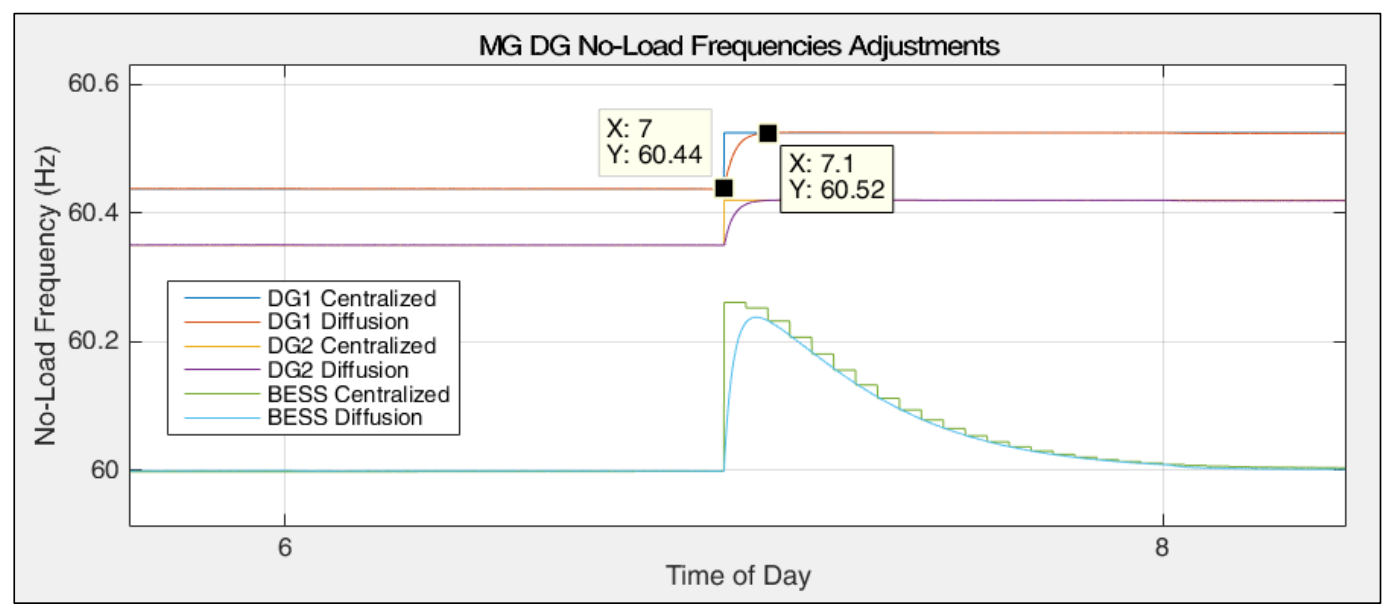

Figure 6.5.11: DER No-Load Frequency Adjustments, Grid-Connected, Close-Up

charge or discharge according to the rate and it settles at an equilibrium point when the rates don’t change (Fig 6.5.12).

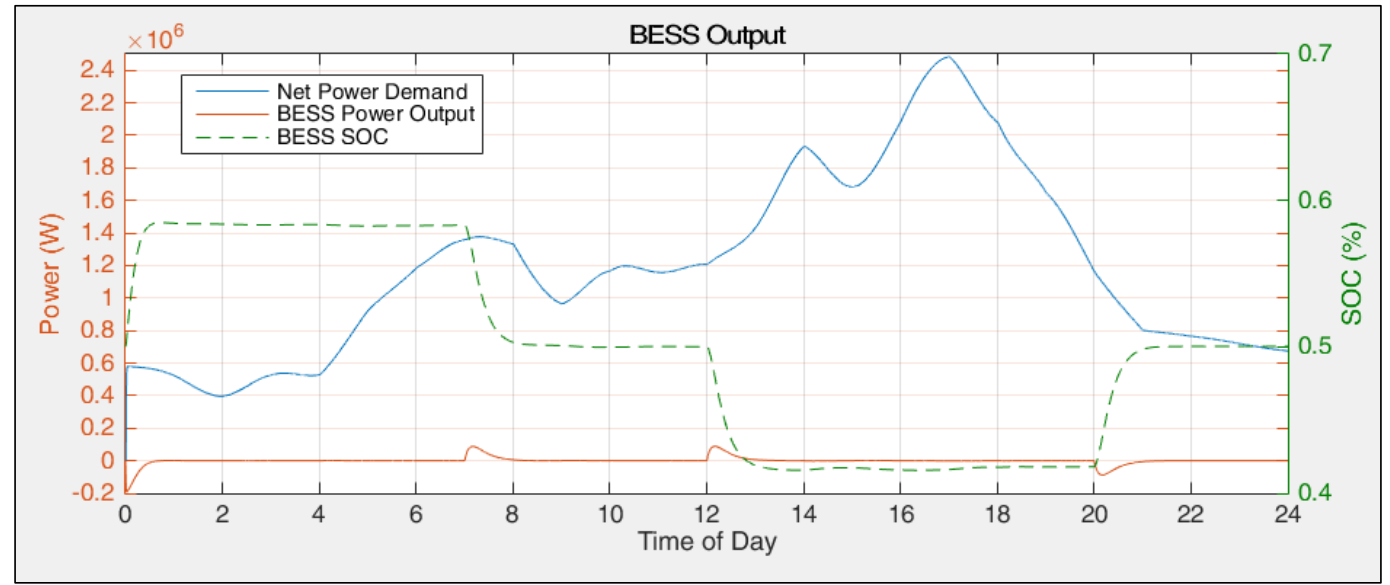

Figure 6.5.12: BESS Power Output vs SOC, Grid-Connected

As explained in Section 4.3, the battery cost function was designed to charge during low-rate periods and discharge during peak hours, which can be clearly observed in Fig. 6.5.12. As was expected, the frequency and voltage deviations are much shorter and only happen during rate transition periods: 


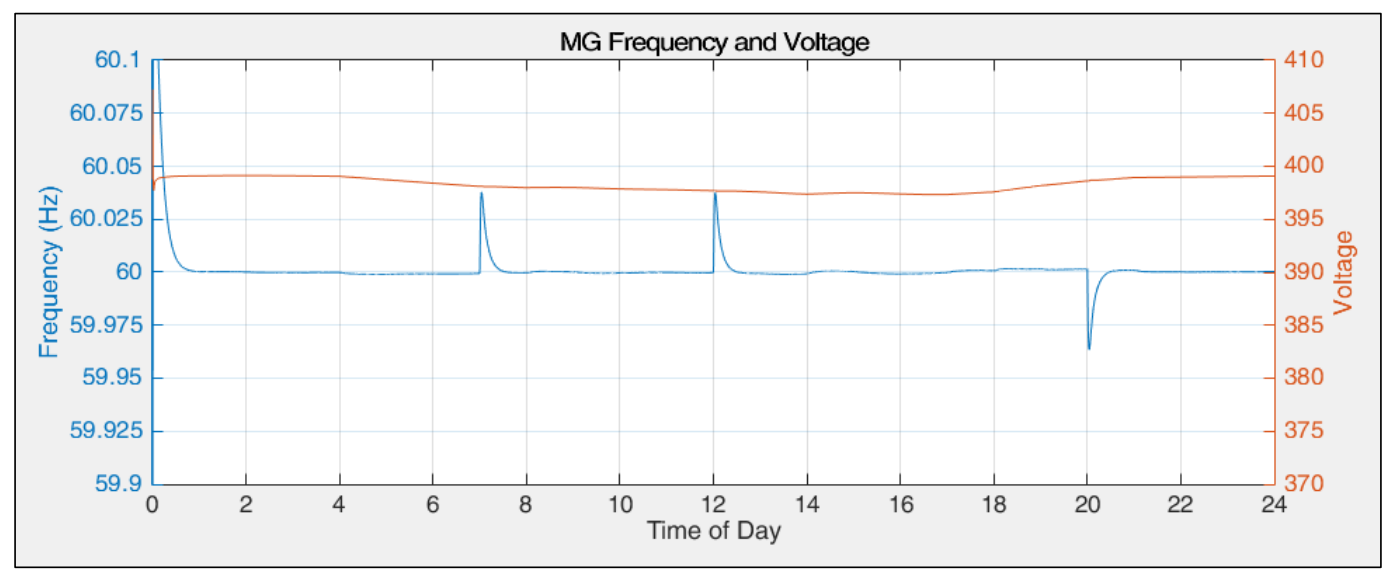

Figure 6.5.13: MG Frequency and Voltage, Grid-Connected

The transient spikes go a bit past the maximum deviations shown in the islanded scenario due to the instantaneous change in marginal cost when the TOU rates change, but the overall frequency and voltage is more stable at the respective nominals of $60 \mathrm{~Hz}$ and 400V, as expected.

\subsubsection{ISLANDING EVENT}

The third scenario to analyze is the case where there is an islanding event, which causes the Point of Common Coupling (PCC) breaker to open and therefore the net demand needs to suddenly be maintained solely by the DERs. Again, the IS diffusion tracks net power, as in the previous scenarios (Fig. 6.5.1 and 6.5.2), but when the islanding event happens, the generators need to quickly adjust to the new operating conditions (Fig. 6.5.14). As seen in the previous grid-connected scenario, the MG is initially selling power to the utility grid. When the islanding event happens at exactly 2 AM, the DGs immediately drop their outputs and the BESS begins charging heavily because the marginal costs dropped significantly due to the relatively low net load demand (Fig. 6.5.15). 


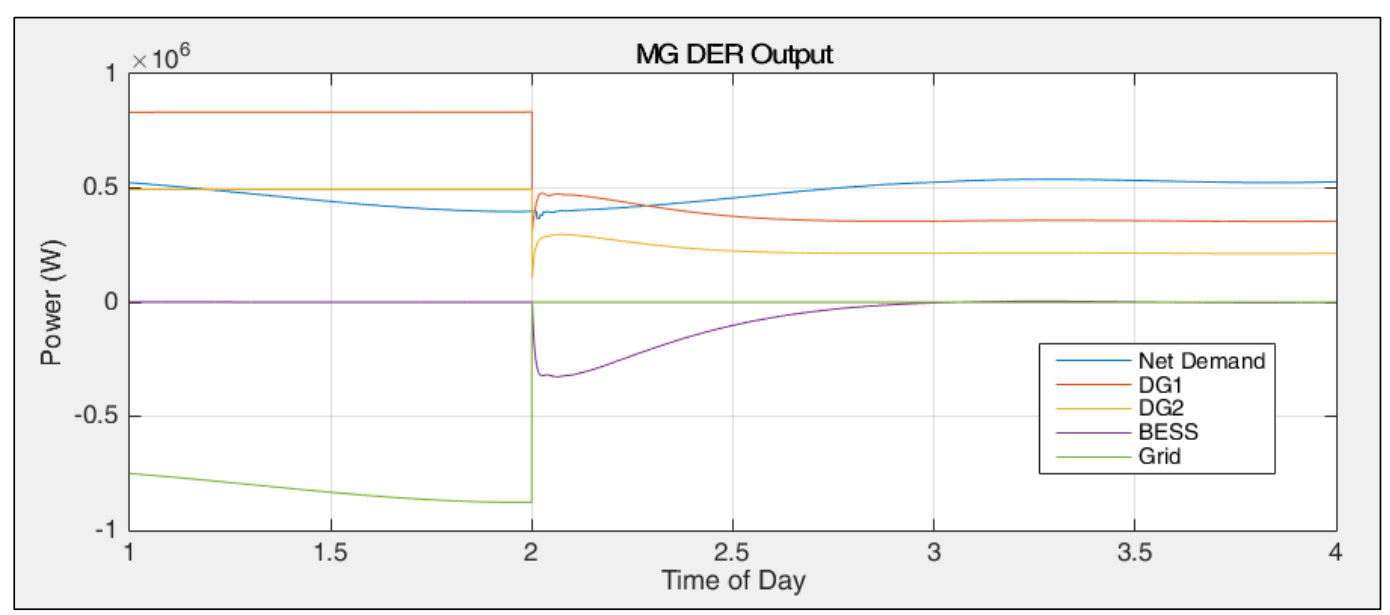

Figure 6.5.14: MG DER Output, Islanding

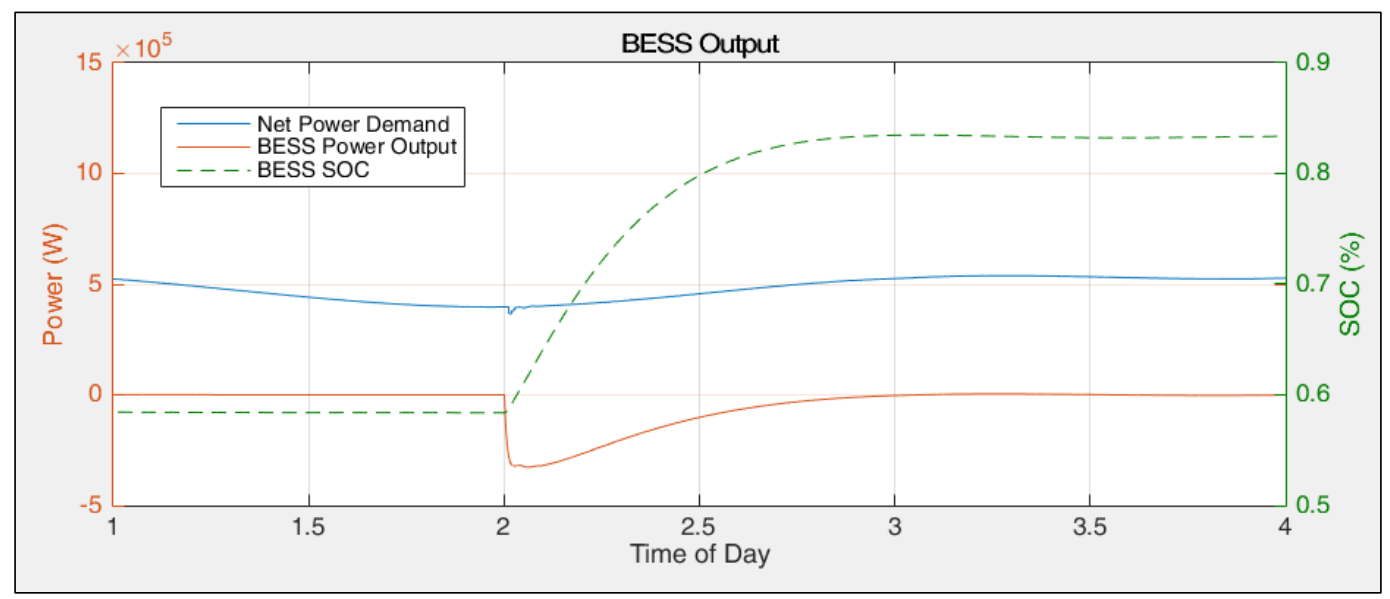

Figure 6.5.15: BESS Power Output vs SOC, Islanding

After the initial transient conditions, the scenario becomes exactly like the first islanded scenario. Because of the abrupt nature of the event, the frequency and voltage exhibit a more dramatic deviation from the nominal (Fig. 6.5.16).

The frequency shoots up to around $60.3 \mathrm{~Hz}$ due to the extra power being generated, as the MG was initially exporting power to the grid. However, the deviation is well within the boundaries established by the IEEE 1547.4-2011 standard [41] (59.3-60.5 Hz are the boundaries). The voltage shoots up further around $10 \%$ up to $440 \mathrm{~V}$. However, it is so short 


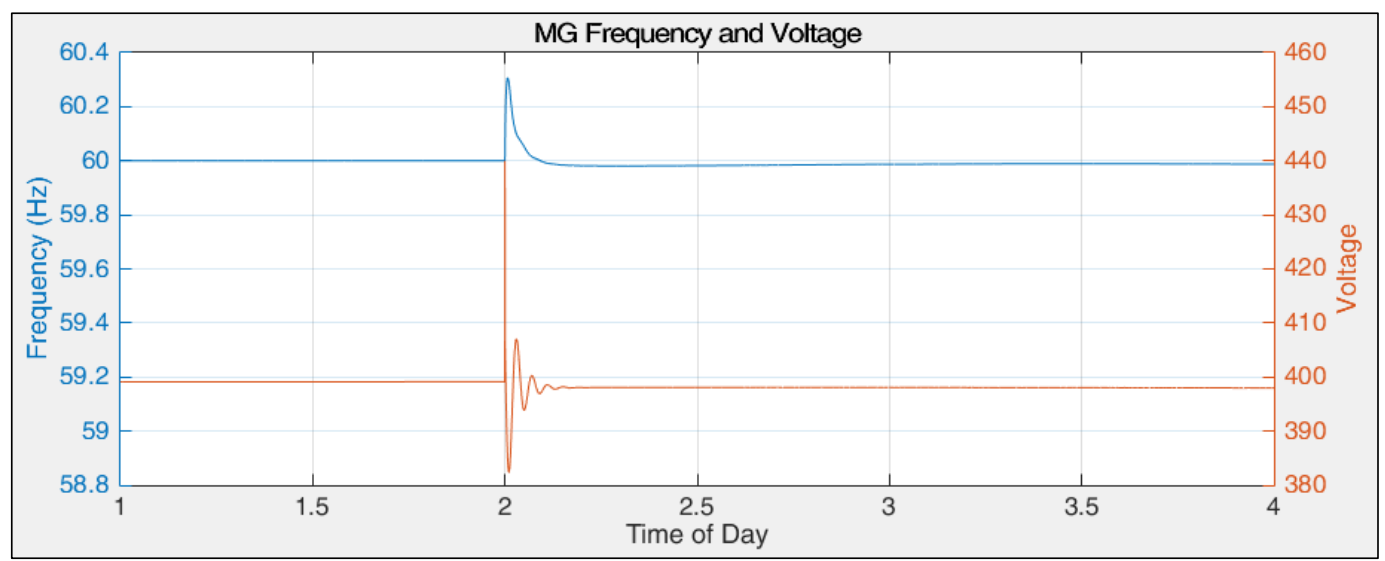

Figure 6.5.16: MG Frequency and Voltage, Islanding

that it wouldn’t violate any standard such as the Information Technology Industry Council (ITIC) Computer and Business Equipment Manufacturers Association's (CBEMA) specification.

This scenario additionally serves to demonstrate the droop control in action; recalling from Chapter 3, droop is used as primary control, which acts immediately and automatically to adjust the DG output according to system frequency. As shown in Fig. 6.5.17, which is a close-up of the droop control mechanism, as soon as the islanding event happens and frequency starts surging, the power set-point of the DG immediately starts dropping. The No-Load Frequency set-point, which is set by the MAS, takes just a bit longer to react and adjust downwards. This demonstrates the stability and effectiveness of a combined control strategy, where the droop control maintains the system stability right away and the MAS then coordinates system-wide and adjusts each asset to its optimum point. 


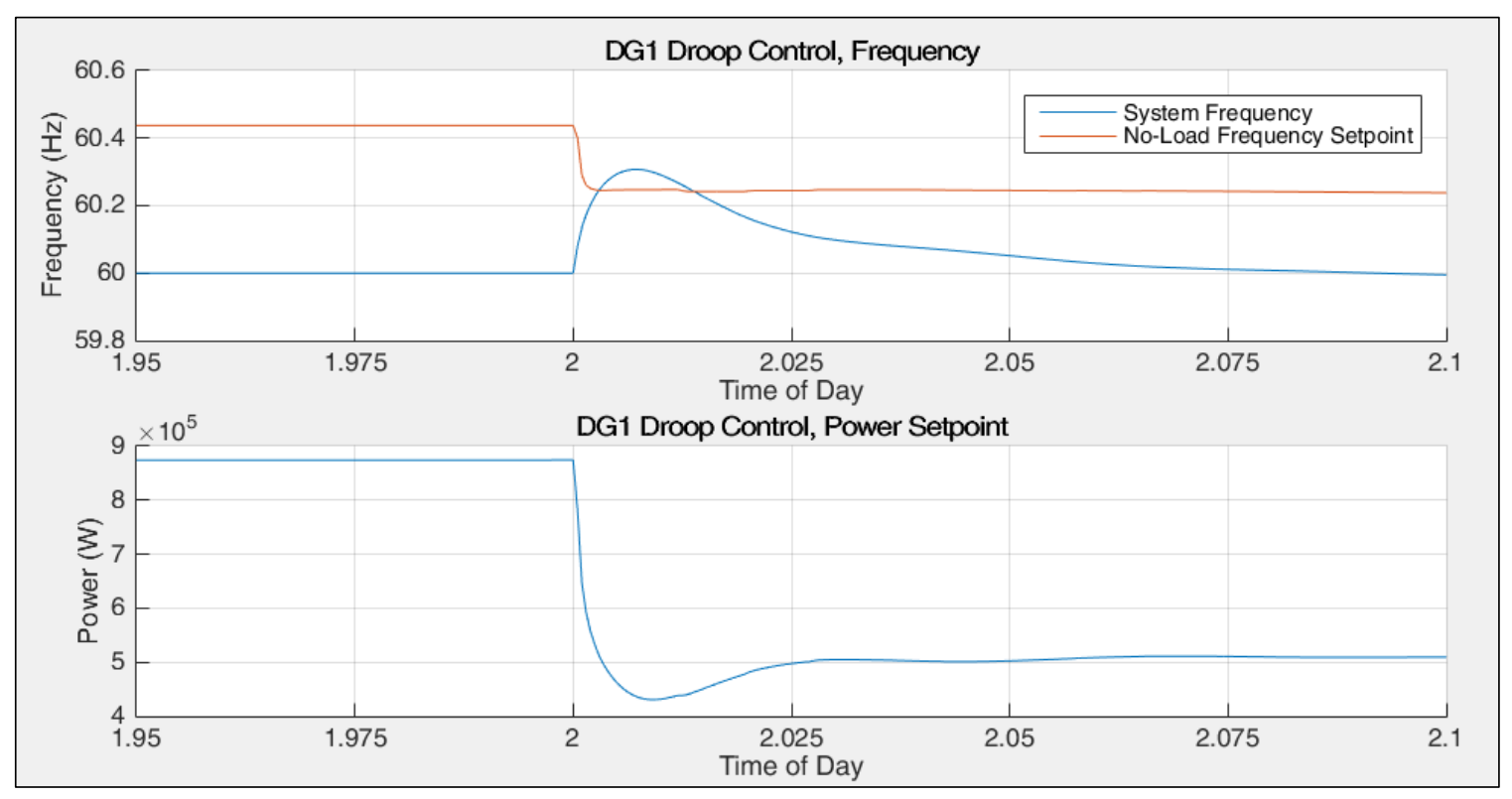

Figure 6.5.17: DG1 Droop Control, Islanding

\subsubsection{SIMULATION CONCLUSION}

With the simulation platform, the concept of using diffusion in a decentralized Multi-Agent system to adjust the Distributed Energy Sources for power imbalances and operate them in an optimal fashion was proven and analyzed. The performance of the modified diffusion algorithm was established and compared to a centralized solution with promising results. The combined droop and MAS control was shown to provide a reliable, effective and flexible solution for future Microgrids.

Next, the JADE implementation described in Section 6.3 will be used in a smart grid testbed to evaluate the performance under real-world conditions, including communication delays, signal noise and an extremely low-inertia power system. 


\subsection{EXPERIMENTAL Platform}

After successfully modeling the proposed platform in a simulation environment, the next step is to test it in a real-world scenario. The Florida International University's Energy Systems Research Laboratory Smart Grid Testbed has all the equipment necessary to conduct such an experiment. The testbed includes Distributed Generators (DGs), Inverter-Based Distributed Energy Resources (DERs), Battery Energy Storage Systems (BESS), transmission and distribution line models, Phasor Measurement Units (PMUs), Intelligent Electronic Devices (IEDs), Programmable Logic Controllers (PLCs), Supervisory Control and Data Acquisition (SCADA) and communication infrastructure. Fig. 6.6.1 shows the Alternating Current (AC) side of the laboratory with the aforementioned components and Fig. 6.6.2 shows the Direct Current (DC) side.

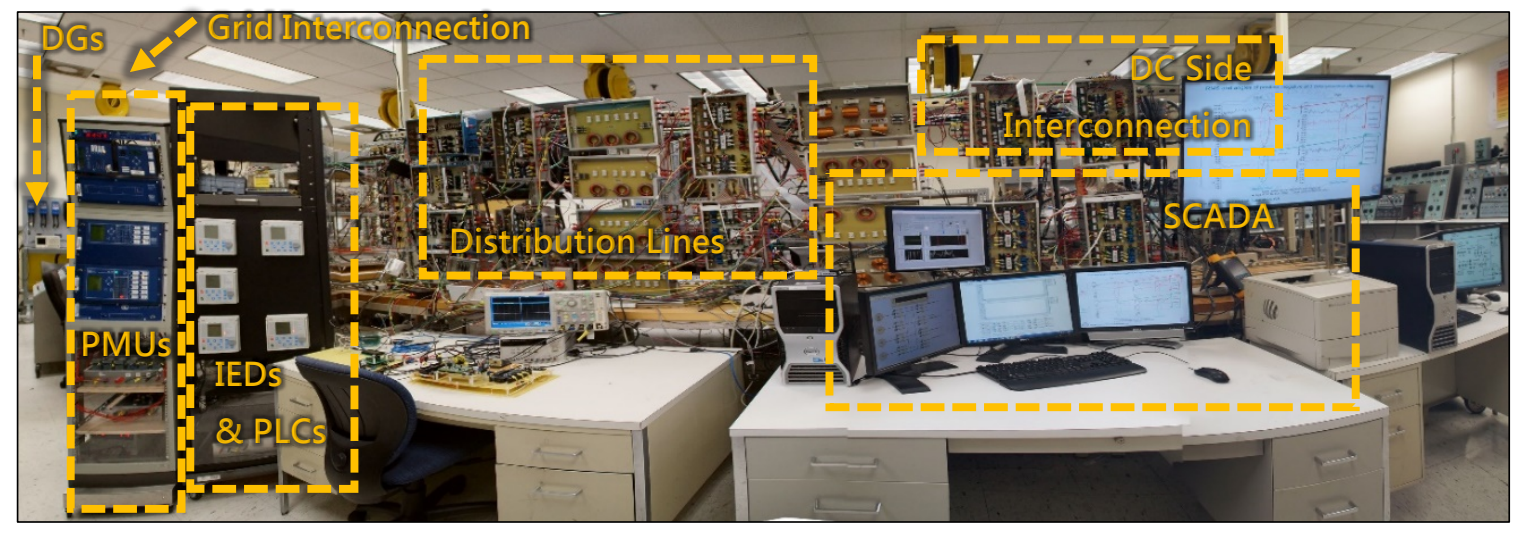

Figure 6.6.1: Smart Grid Testbed, Alternating Current Side

As previously stated in Section 6.3, the experimental Microgrid (MG) consists of five nodes; two DGs, one BESS, and two loads. The experiment was run on an islanded MG to test the MAS control on a low-inertia system (Fig. 6.6.3). 


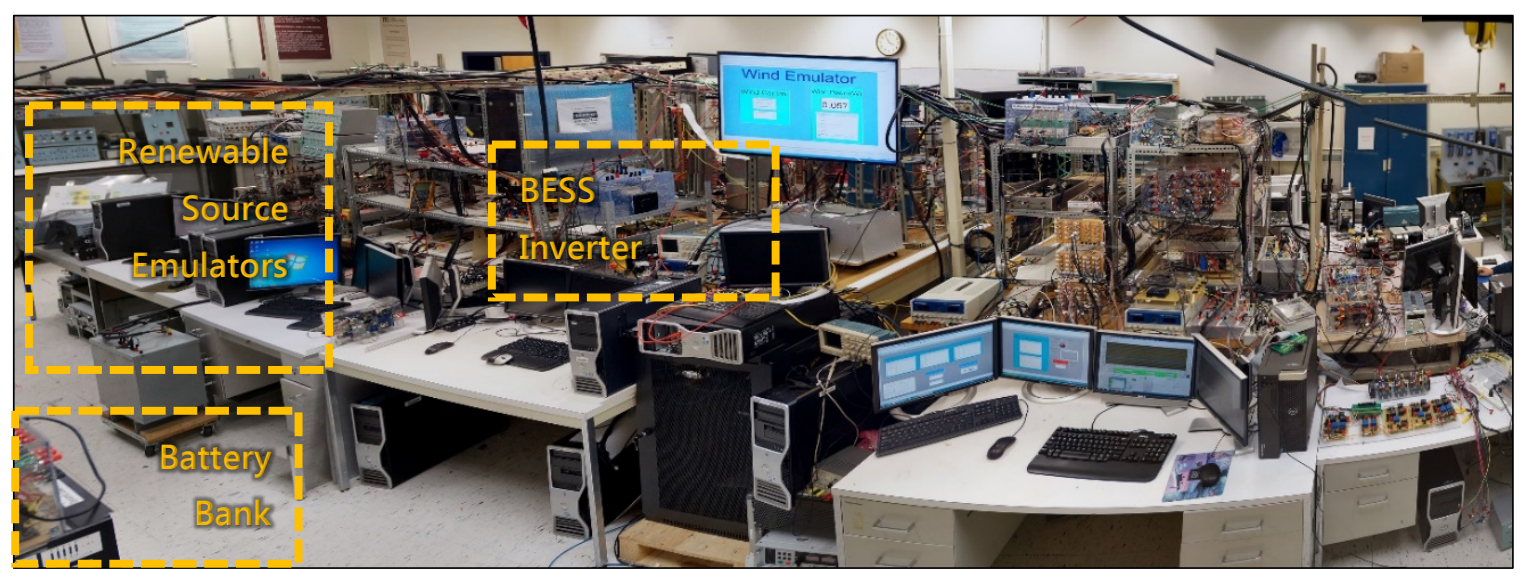

Figure 6.6.2: Smart Grid Testbed, Direct Current Side

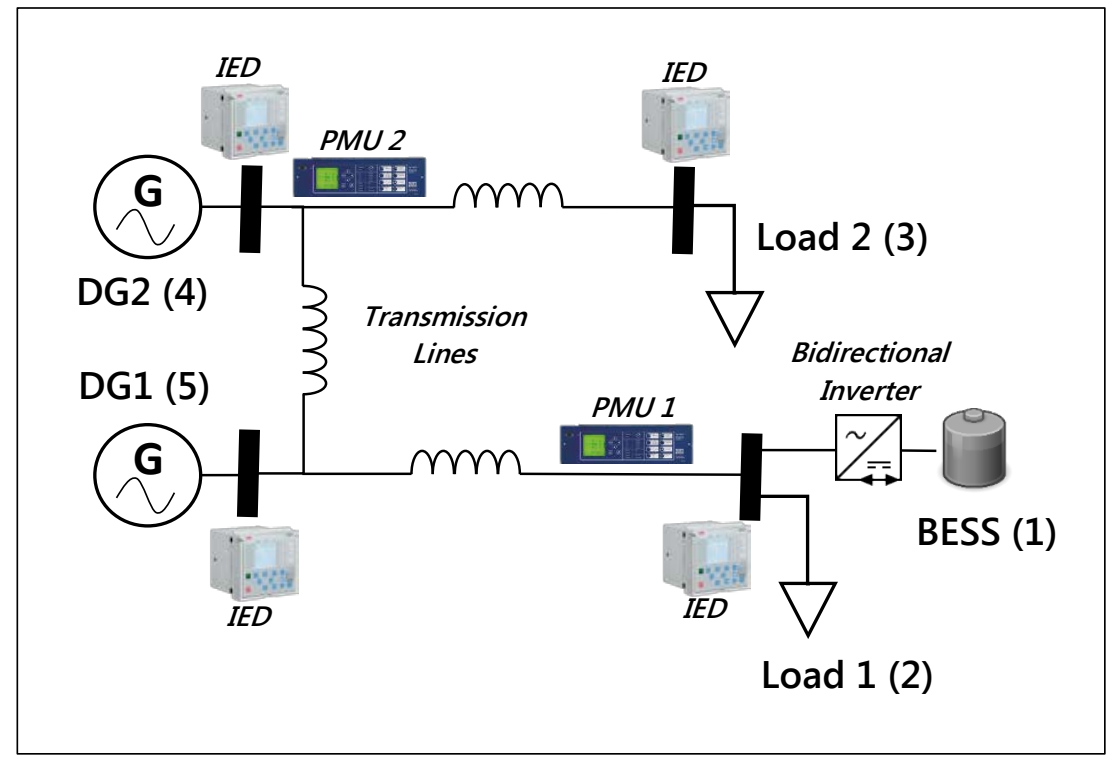

Figure 6.6.3: Experimental MG Physical Layout

Node 1 of the experimental MG is the BESS, which sits on the DC side of the testbed (Fig. 6.6.2). It is composed of a battery emulator (DC source) and a $1 \mathrm{~kW}$ threephase bidirectional inverter, which synchronizes to the AC side and can inject or withdraw power from the MG. Node 2 is a controllable three-phase load, which is connected on the same bus as the BESS; this is the load that will suddenly increase when the experiment runs, as explained in Section 6.7. Nodes 1 and 2 connect through a transmission line to 
DG1, which is labeled node 5; it is a $5 \mathrm{~kW}$ three-phase synchronous generator. DG1 connects to DG2 on node 4 through another transmission line; it is a similar but smaller generator with a rating $3 \mathrm{~kW}$. DG2 connects through yet another transmission line to a second controllable three-phase load at node 3.

Obviously, every system is going to be quite different and each electrical component will have its own characteristics and control interface. For this experiment, the MAS control scheme had to be adapted to the equipment available in the lab. Because the two DGs are of such a low rating, they provide very limited inertia to the system and their control is quite erratic. The DGs are controlled through serial communication ports with torque references ranging from 0-100. Although not very precise, each additional torque step for DG1 represents around 52 Watts of output power and $38 \mathrm{~W}$ for DG2. A mapping of torque vs output power was done in order to program the agents to provide the torque values closest to the optimized power points reached by the diffusion algorithm:

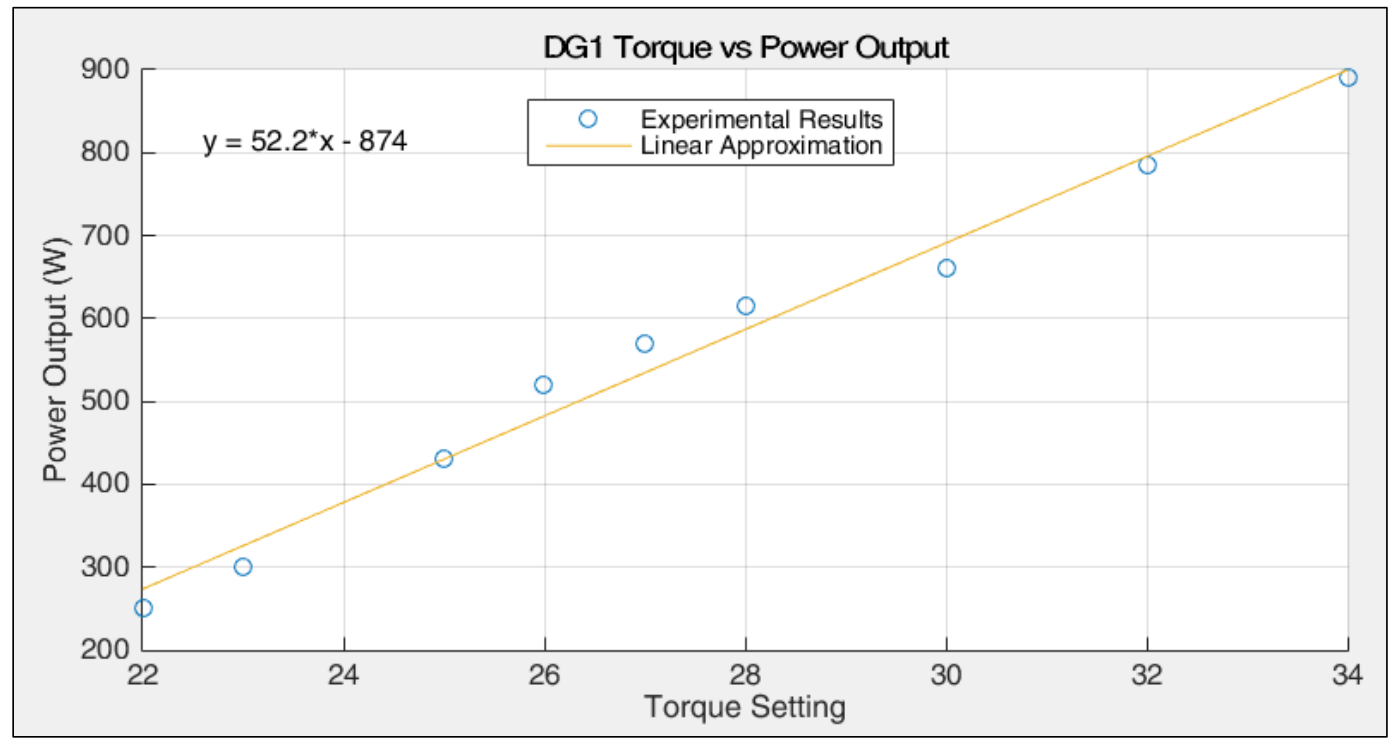

Figure 6.6.4: DG1 Torque vs Power Output 


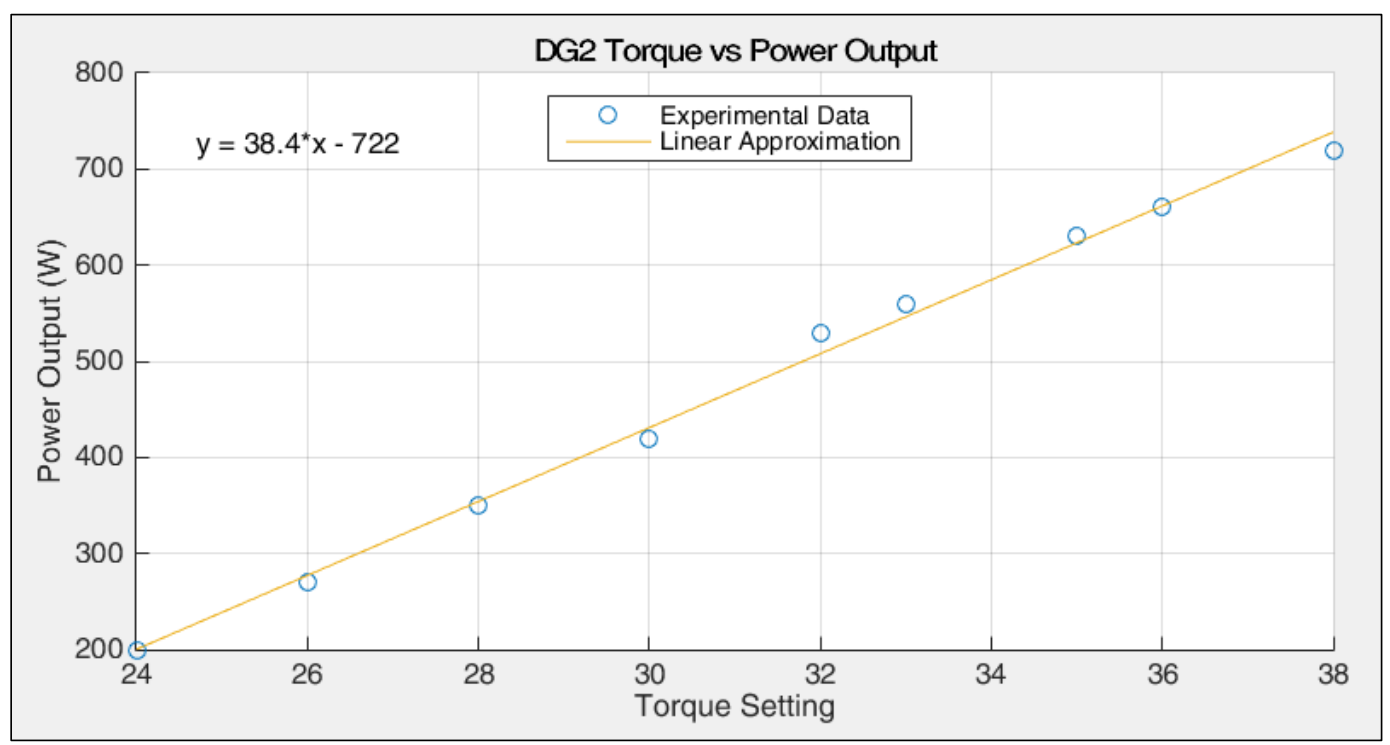

Figure 6.6.5: DG2 Torque vs Power Output

The BESS provides a more precise control, given that the inverter is an advanced power electronics device with the ability to accurately determine the current and voltage output. The BESS can run in two modes: it can be used as a slack DER to provide the power necessary to maintain nominal frequency, or it can be used as a current injector independent of the system frequency.

PMUs 1 and 2 are actually located together, as shown in Fig. 6.6.1, but they are able to monitor several electrical connections at a time. PMU 1 measures the output from the BESS inverter and DG1, while PMU 2 measures the output from DG2. Additionally, they measure the voltage and frequency of the system and store it in a local database, which can be queried to obtain detailed information about the overall behavior of the grid or a specific event. Also, there are IEDs at every bus that implement overcurrent protection and similarly provide measurements.

The aforementioned measurements from the PMUs and IEDs are communicated throughout the MG via OPC middleware. OPC stands for Open Platform Communications, 
which was originally developed as OLE (Object Linking and Embedding) for Process Control [42]. It is a standard for secure and reliable communication for industrial telecommunications within different platforms. The communicational topology of the MG, which was arbitrarily chosen, is shown in Fig. 6.6.6:

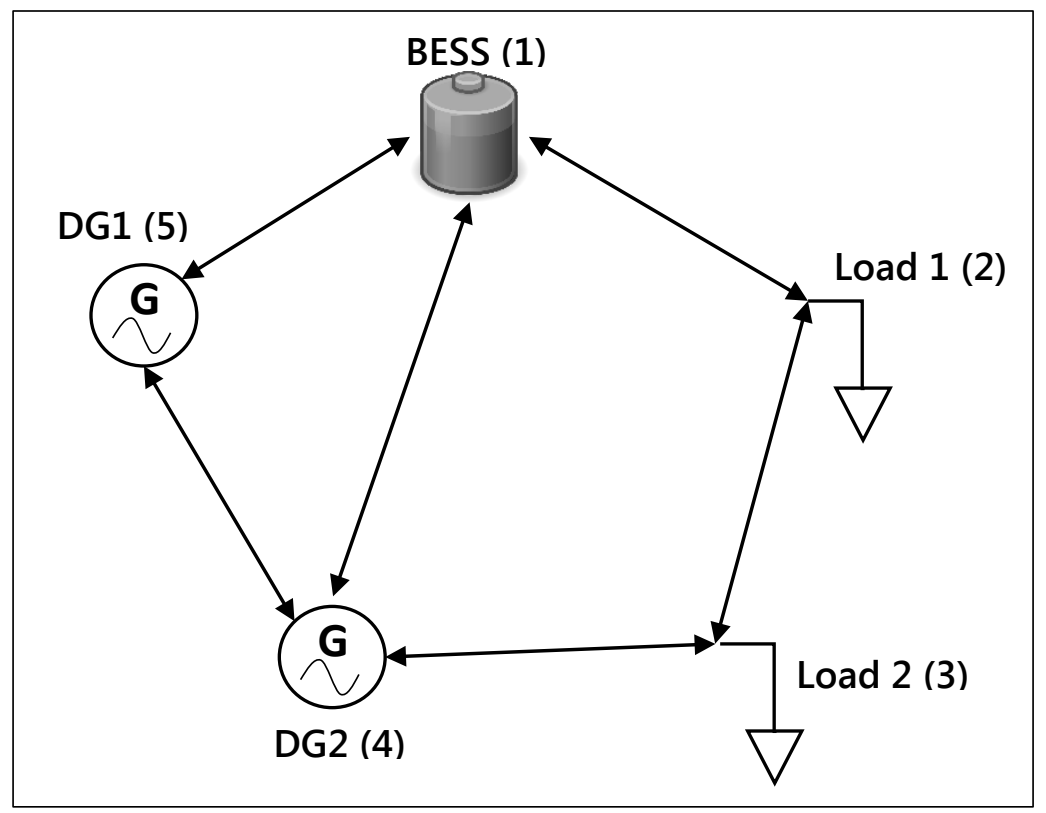

Figure 6.6.6: Experimental MG Network Topology

The adjacency matrix for this network topology using Metropolis weights (including self-connections for each agent) is:

$$
A=\left\{\begin{array}{rrrrr}
0.25 & 0.25 & 0 & 0.25 & 0.25 \\
0.25 & 0.42 & 0.33 & 0 & 0 \\
0 & 0.33 & 0.42 & 0.25 & 0 \\
0.25 & 0 & 0.25 & 0.25 & 0.25 \\
0.25 & 0 & 0 & 0.25 & 0.50
\end{array}\right\}
$$

As described in Section 6.3, and illustrated in Fig. 6.3.1, there is a JADE agent for each of the nodes in the MG. The weights from the $A$ matrix are multiplied to the estimates coming from each of the neighbors, as explained in Chapter 5 . The agents use a Java plugin 
to send and receive data to and from the OPC server. The stochastic agents (loads) read the current power being drawn from the MG via the IEDs; this would be the primary layer, as shown in Fig. 6.2.1. The dispatchable agents (DGs and BESS) in turn write the power points of their respective assets to the OPC server, which is then picked up by a LabVIEW SCADA system that communicates through a serial port connection to the DERs. Again, this is analogous to the primary control layer for dispatchable agents, as described in Fig. 6.2.2.

\subsection{EXPERIMENTAL RESULTS}

To start the MG and take it to steady state in order for the experiment to run, there are several steps that need to happen first. Initially, DG1 is started with a small load on node 2. DG2 is started on its own and synchronized to DG1 through a Phase-Locked Loop (PLL) controller with a dynamic break resistor, which can rapidly vary its resistance to accelerate or decelerate the generator in order to get it in synch with the system. When the DGs are synchronized, a remote breaker is activated by the IED to connect both DGs. The BESS is similarly synchronized through an electronic PLL, which detects the system frequency and adjusts the current output of the inverter to match it.

Once all DERs are synchronized, they are brought to their steady state output values: DG1 generates around $740 \mathrm{~W}, \mathrm{DG} 2$ around $600 \mathrm{~W}$ and the BESS around $50 \mathrm{~W}$; load 1 is consuming around $600 \mathrm{~W}$ and load 2 around $800 \mathrm{~W}$. The JADE agents have reached an equilibrium through diffusion optimization in terms of the Economic Dispatch (ED) point of the DERs with the given load. Suddenly, $300 \mathrm{~W}$ of additional load are added to load 1 at node 2 of the MG. Soon after, the Information Sharing (IS) diffusion notifies 
all agents about the load increase and the power points are subsequently adjusted. After the initial readjustments, the diffusion optimization continues to run, and after a few seconds, new ED points are reached. The following figures show the behavior of the MG before, during and after the loading event:

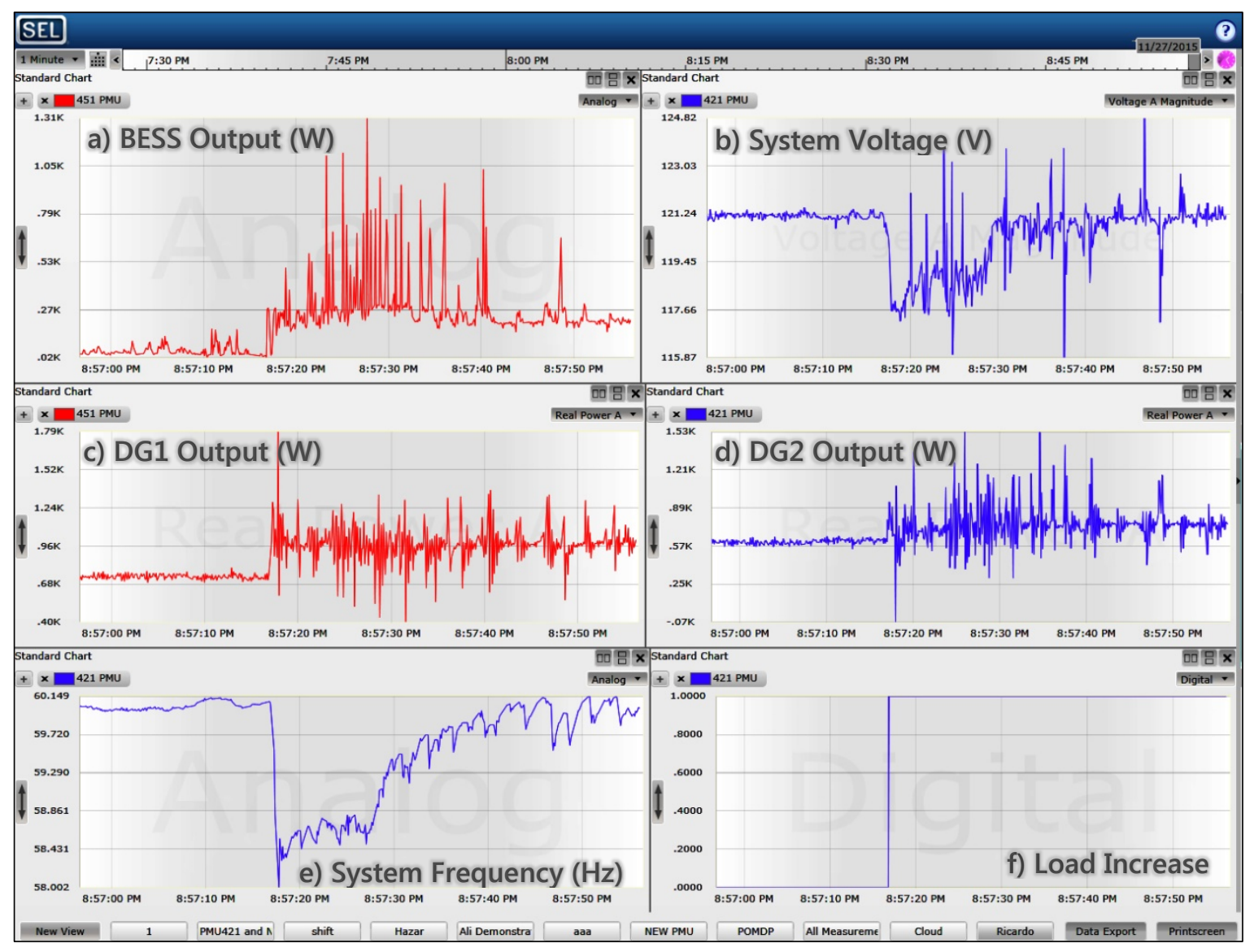

Figure 6.7.1: PMU Experimental Output

Fig. 6.7.1 shows the measurements taken from the Phasor Data Concentrator (PDC), which is a monitoring tool for the data from the PMUs. Subfigure a) shows the output of the BESS, b) the system voltage, c) DG1 output, d) DG2 output, e) the system frequency and f) shows the exact moment when the additional load was enabled. As can be observed, the measurements are quite noisy and it is hard to determine the actual output of the DERs. 
However, the data can be exported onto files for further processing and analysis; MATLAB software is used to apply a moving average low pass filter:

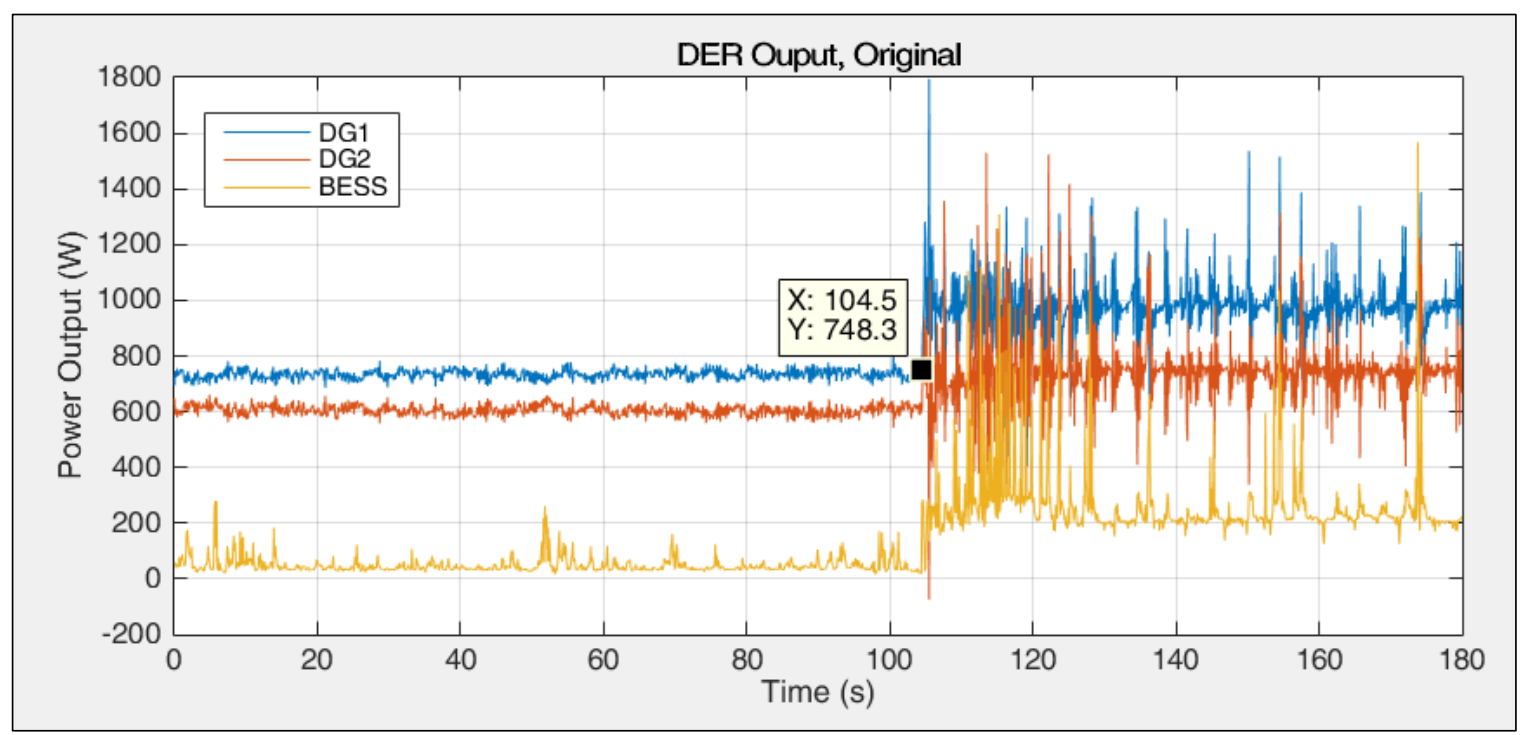

Figure 6.7.2: Experimental DER Output, Original

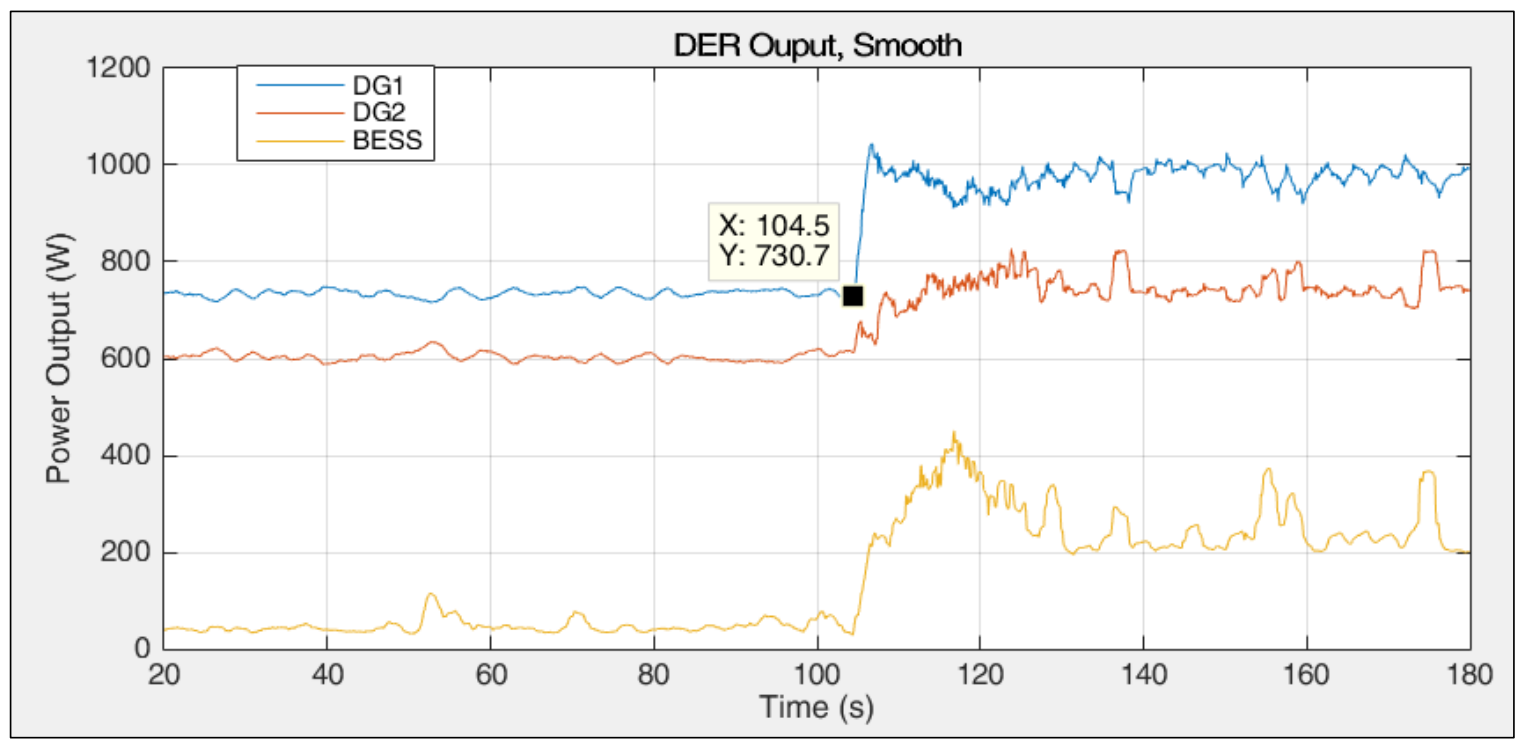

Figure 6.7.3: Experimental DER Output, Filtered

Fig. 6.7.2 shows 180 seconds of the raw imported data from the PMU; again, the noisy nature of the data can be perceived. Fig. 6.7.3 shows the filtered data, which exposes 
quite a different picture. As indicated on both figures, at 104.5s, the load is activated and the DERs' droop control immediately respond. DG1 initially takes most of the load since it is the largest DER, and the BESS also responds very quickly because of the fast-acting power electronics. The power points are adjusted quickly after the new load is added by the diffusion algorithm and then readjusted by the diffusion optimization. Looking at the frequency, along with the power, provides another angle to the story:

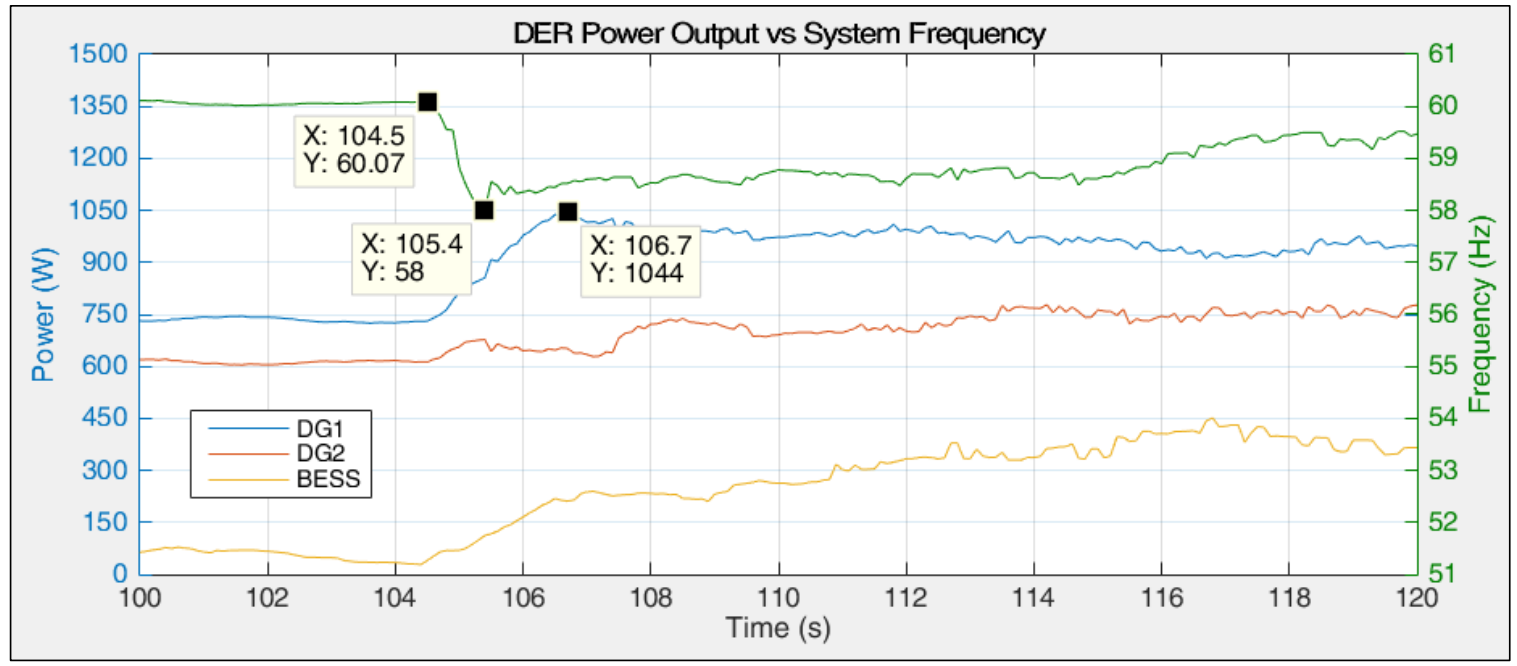

Figure 6.7.4: Experimental DER Output vs Frequency, Close-Up

Fig. 6.7.4 shows a close-up of around 5 seconds prior to the event and 15 seconds afterward. Again, at 104.5s, the moment the additional load is switched on and the frequency starts declining is observed. The droop control immediately kicks in and the DER outputs start rising; by 105.4s (900 milliseconds afterward), the frequency reaches its minimum at $58 \mathrm{~Hz}$. Meanwhile, the MAS with diffusion communication has adjusted the power points of the DERs as secondary frequency control and both DG1 and the BESS keep rising in output until 106.7s; DG2 is maintained about the same after the initial droop response. Secondary and tertiary frequency control continue to bring the frequency back 
up and readjust the power outputs to their optimal points. DG1 is brought down a bit and DG2 exhibits a slight uptick at around 107s; the BESS is continually rising until around 117s. Zooming out a bit, the decentralized secondary and tertiary control is further observed:

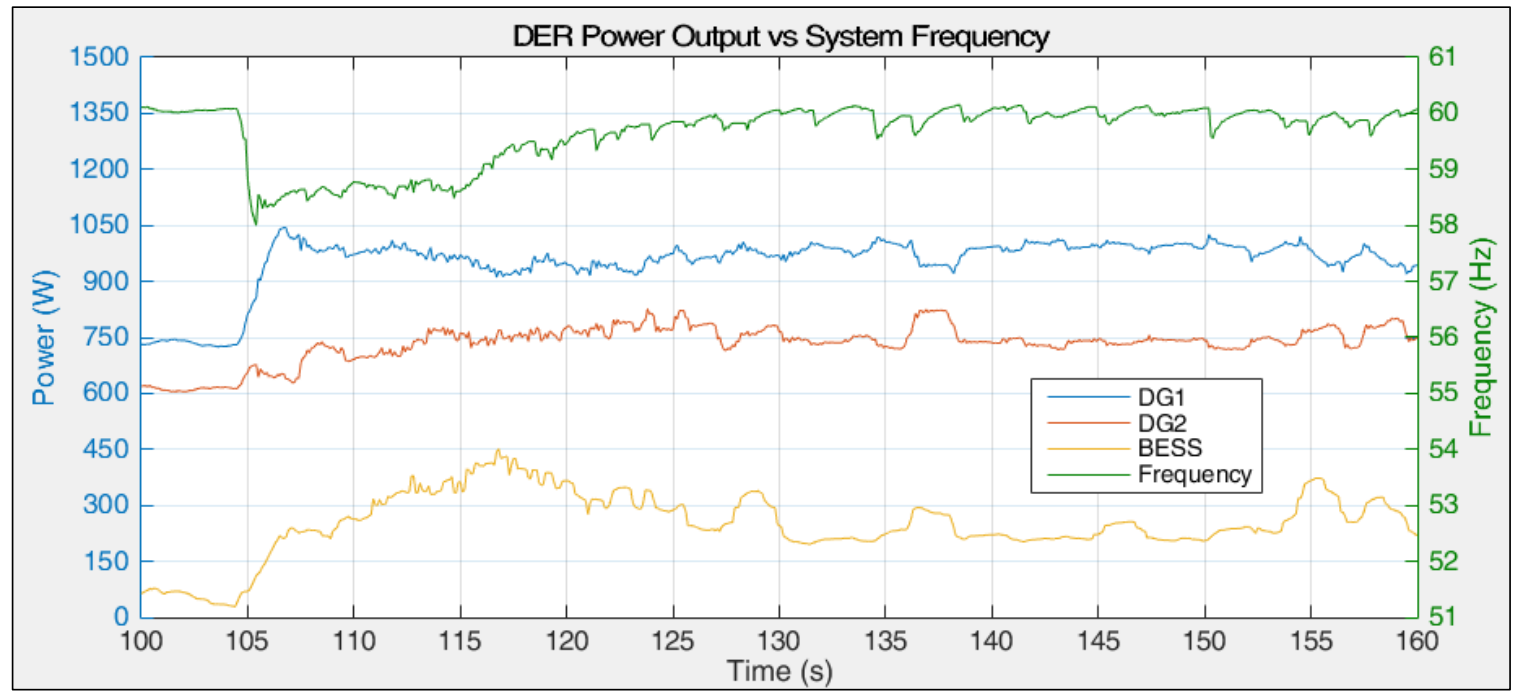

Figure 6.7.5: Experimental DER Output vs Frequency, Zoom-Out

After the initial drop in frequency and the increase in power from the droop control, the subsequent adjustments being done by the MAS are observed. At around 125s, the frequency is fully restored to nominal, but the optimization continues to happen. After the initial rise of the BESS from around $50 \mathrm{~W}$ to almost $450 \mathrm{~W}$, it is then adjusted downward and starts to settle around $200 \mathrm{~W}$. DG2, which started around $600 \mathrm{~W}$ initially, rose to around $675 \mathrm{~W}$ with droop and started to settle around $750 \mathrm{~W}$ towards the end. DG1, which started around $750 \mathrm{~W}$, shot up to around $1050 \mathrm{~W}$ with droop and secondary control, then started to settle at around $975 \mathrm{~W}$. 
The choppy behavior in frequency after the system has recovered is due to the ED adjustments, but as seen in Fig. 6.7.6, the variations are within the standards (59.3-60.5 $\mathrm{Hz})$ :

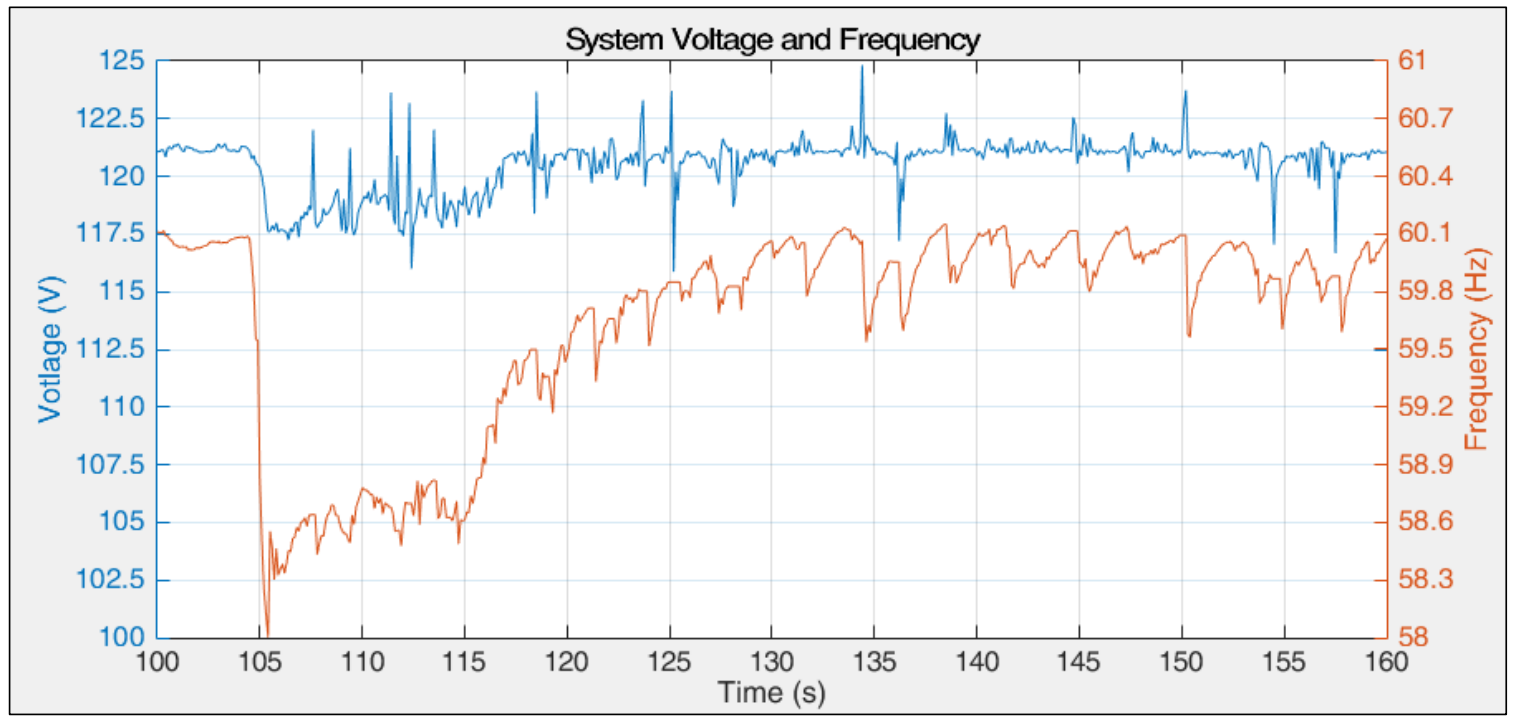

Figure 6.7.6: Experimental System Voltage and Frequency

The tertiary adjustments after the frequency has recovered to nominal maintain the frequency above $59.5 \mathrm{~Hz}$. Although the initial dip to $58 \mathrm{~Hz}$ violates the IEEE 1547.4-2011 standard, given the extremely low inertia present in the system, the results are actually quite remarkable. A real-world MG would have orders of magnitude more rating and inertia, and the proposed control system would more easily keep the values within the standards. The voltage is also kept within 5 volts of the $120 \mathrm{~V}$ nominal, which is within CBEMA standards. 


\section{CHAPTER 7: CONCLUSION AND RECOMMENDATION FOR FUTURE WORK}

\subsection{CONCLUSION}

The purpose of this thesis was to formulate, develop, simulate and test a comprehensive control system for a Microgrid (MG), which is fully decentralized and operates optimally. Initially, the justification for the work and the background necessary to understand the proposed system was explained. The need for, and progress towards, distributed control schemes was described, as well as the concept of Multi-Agent Systems (MAS). Information sharing in networks was introduced and diffusion was presented, as well as a method for decentralized optimization. Finally, all the concepts were put together in an all-inclusive control system, which was successfully simulated and tested.

The simulation platform provides the ability to assess the system under many scenarios and precisely compares the performance versus existing schemes. It was shown how the system stability was maintained even under emergency circumstances and how the efficiency of the MG was sustained without a complex centralized controller infrastructure. The experimental platform provided the ability to evaluate the MAS under real-world conditions and ultimately prove its viability.

The work represents an excellent starting point for a scheme, which could be fully

developed and implemented in real world MGs. As explained in Section 7.2, suggested future work will be outlined in order to take the concept to market.

A summary of the contributions in this thesis are presented:

- A novel cost function for an Energy Storage System (ESS), which is agnostic to the type of system and automatically charges when the rate of electricity (or marginal cost) is low and discharges when high, is described in Section 4.3. 
- Diffusion for information sharing in a MG is presented in Section 5.2, and in Section 5.4, an innovative variation of distributed optimization for MGs using diffusion is formulated and tested.

- In Section 6.2, an original agent structure for the MG MAS is outlined and explained.

- A unique implementation of the MG MAS using the Java Agent DEvelopment Framework (JADE) is defined in Section 6.3.

- The experimental platform and results are presented in Sections 6.6 and 6.7; to the best of the author's knowledge, such a system has never been experimentally tested in the literature before.

\subsection{FUTURE WORK}

As with any academic research work, there are many things to be expanded on; here a few concepts are suggested:

- Implement machine learning into the agents for load and renewable energy forecasting, as well as Energy Storage optimization with accurate economic forecasts.

- Include Electric Vehicle (EV) agents, which are mobile and therefore plug in and out of the MG at different places. A stochastic and probabilistic model could be implemented to simulate the behavior of these agents under different MG topologies and numbers of EVs present in the system.

- Develop decentralized load shedding schemes for islanded MGs when the demand surpasses local DER supply capabilities. 
- Further investigate the communication infrastructure and security implications.

- Implement electric losses and ramp rates into models.

Given that the work done represents academic research and not product development, there are a few steps to be taken to carry the proposed system to market:

- Further testing of different network topologies and operating conditions.

- Verification and fine-tuning of DER cost functions to closely match real-world systems.

- Researching and implementing communication infrastructures for real-world MGs.

- Finally, implementing the agents on embedded hardware systems for the different types of devices. This is the step that needs the most work in the author's opinion because the electronics need to be developed for, and tested on, many different apparatuses. However, once they are deployed, the devices would self-regulate, and the network could be expanded or re-designed without much further work. 


\section{REFERENCES}

[1] N. Hatziargyriou, H. Asano, R. Iravani and C. Marnay, "Microgrids," Power and Energy Magazine, IEEE, vol. 5, no. 4, pp. 78-94, 2007.

[2] R. Lasseter, "MicroGrids," Power Engineering Society Winter Meeting. IEEE, vol. 1, pp. 305-308, 2002.

[3] J. Guerrero, M. Chandorkar, T. Lee and P. Loh, "Advanced Control Architectures for Intelligent Microgrids_Part I: Decentralized and Hierarchical Control," Industrial Electronics, IEEE Transactions on, vol. 60, no. 4, pp. 1254-1262, 2013.

[4] R. Olfati-Saber, J. Fax and R. Murray, "Consensus and Cooperation in Networked Multi-Agent Systems," Proceedings of the IEEE, vol. 95, no. 1, pp. 215-233, 2007.

[5] A. Sayed, "Adaptive Networks," Proceedings of the IEEE, vol. 102, no. 4, pp. 460497, 2014.

[6] J. Chen and A. Sayed, "Diffusion Adaptation Strategies for Distributed Optimization and Learning Over Networks," Signal Processing, IEEE Transactions on, vol. 60, no. 8, pp. 4289-4305, 2012.

[7] J. Chen and A. Sayed, "Distributed Pareto Optimization via Diffusion Strategies," Selected Topics in Signal Processing, IEEE Journal of, vol. 7, no. 2, pp. 205220, 2013.

[8] S.-Y. Tu and A. Sayed, "On the Influence of Informed Agents on Learning and Adaptation Over Networks," Signal Processing, IEEE Transactions on, vol. 61, no. 6, pp. 1339-1356, 2013.

[9] V. Salehi, A. Mohamed, A. Mazloomzadeh and O. Mohammed, "'Laboratory-Based Smart Power System, Part I: Design and System Development," Smart Grid, IEEE Transactions on, vol. 3, no. 3, pp. 1394-1404, 2012.

[10] V. Salehi, A. Mohamed, A. Mazloomzadeh and O. Mohammed, "Laboratory-Based Smart Power System, Part II: Control, Monitoring, and Protection," Smart Grid, IEEE Transactions on, vol. 3, no. 3, pp. 1405-1417, 2012.

[11] W. Shi, X. Xie, C.-C. Chu and R. Gadh, "Distributed Optimal Energy Management in Microgrids," Smart Grid, IEEE Transactions on, vol. 6, no. May, pp. 11371146, 2015. 
[12] A. Parisio, E. Rikos and L. Glielmo, "A Model Predictive Control Approach to Microgrid Operation Optimization," Control Systems Technology, IEEE Transactions on, vol. 22, no. 5, pp. 1813-1827, 2014.

[13] M. Shadmand and R. Balog, "Multi-Objective Optimization and Design of Photovoltaic-Wind Hybrid System for Community Smart DC Microgrid," Smart Grid, IEEE Transactions on, vol. 5, no. 5, pp. 2635-2643, 2014.

[14] K. Luo and Wenhui Shi, "Distributed coordination method of microgrid economic operation optimization based on multi-agent system," Power System Technology (POWERCON), International Conference on, pp. 3135-3140, 2014.

[15] C. Colson and M. Nehrir, "Comprehensive Real-Time Microgrid Power Management and Control With Distributed Agents," Smart Grid, IEEE Transactions on, vol. 4, no. 1, pp. 617-627, 2013.

[16] Y. Xu, W. Zhang, W. Liu, X. Wang, F. Ferrese, C. Zang and H. Yu, "Distributed Subgradient-Based Coordination of Multiple Renewable Generators in a Microgrid," Power Systems, IEEE Transactions on, vol. 29, no. 1, pp. 23-33, 2014.

[17] W. Liu, W. Gu, W. Sheng, X. Meng, Z. Wu and W. Chen, "Decentralized Multi-Agent System-Based Cooperative Frequency Control for Autonomous Microgrids With Communication Constraints," Sustainable Energy, IEEE Transactions on, vol. 5, no. 2, pp. 446-456, 2014.

[18] V. Loia, A. Vaccaro and K. Vaisakh, "A Self-Organizing Architecture Based on Cooperative Fuzzy Agents for Smart Grid Voltage Control," Industrial Informatics, IEEE Transactions on, vol. 9, no. 3, pp. 1415-1422, 2013.

[19] L. Meng, T. Dragicevic, J. Guerrero and J. Vasquez, "Dynamic consensus algorithm based distributed global efficiency optimization of a droop controlled DC microgrid," Energy Conference (ENERGYCON), IEEE International, pp. 1276-1283, 2014.

[20] Y. Xu and Z. Li, "Distributed Optimal Resource Management Based on the Consensus Algorithm in a Microgrid," Industrial Electronics, IEEE Transactions on, vol. 62, no. 4, pp. 2584-2592, 2015.

[21] G. Hug, S. Kar and C. Wu, "Consensus + Innovations Approach for Distributed Multiagent Coordination in a Microgrid," Smart Grid, IEEE Transactions on, vol. 6, no. 4, pp. 1893-1903, 2015.

[22] G. M. Masters, Renewable and Efficient Electric Power Systems, Hoboken: Wiley, 2004. 
[23] D. o. Energy, "Smart Grid," [Online]. Available: http://energy.gov/oe/services/technology-development/smart-grid.

[24] A. Mohamed, V. Salehi, T. Ma and O. Mohammed, "Real-Time Energy Management Algorithm for Plug-In Hybrid Electric Vehicle Charging Parks Involving Sustainable Energy," Sustainable Energy, IEEE Transactions on, pp. 577-586, 2014.

[25] B. Lasseter, "Microgrids [distributed power generation]," Power Engineering Society Winter Meeting, IEEE, pp. 146-149, 2001.

[26] C. Marnay, F. Robio and A. Siddiqui, "Shape of the microgrid," Power Engineering Society Winter Meeting, IEEE, pp. 150-153, 2001.

[27] J. D. Glover, M. S. Sarma and T. J. Overbye, Power System Analysis and Design, Stamford: Cengage, 2010.

[28] A. J. Wood, B. Wollenberg and S. G.B., Power Generation, Operation, and Control, Hoboken: Wiley, 2014.

[29] R. Cosse, M. Alford, M. Hajiaghajani and E. Hamilton, "Turbine/generator governor droop/isochronous fundamentals - A graphical approach," Petroleum and Chemical Industry Conference (PCIC), 2011 Record of Conference Papers Industry Applications Society 58th Annual IEEE, pp. 1-8,19-21, 2011.

[30] G. Antonelli, "Interconnected dynamic systems: An overview on distributed control," Control Systems, IEEE, pp. 76-88, 2013.

[31] W. Ren, R. Beard and E. Atkins, "Information consensus in multivehicle cooperative control," Control Systems, IEEE, pp. 71-82, 2007.

[32] Y. Shoham and K. Leyton-Brown, MULTIAGENT SYSTEMS: Algorithmic, GameTheoretic, and Logical Foundations, masfoundations.org, 2010.

[33] N. Li, L. Chen and S. Low, "Optimal demand response based on utility maximization in power networks," in Power and Energy Society General Meeting, 2011 IEEE, 2011.

[34] J. Chen and A. Sayed, "On the limiting behavior of distributed optimization strategies," in Communication, Control, and Computing (Allerton), 2012 50th Annual Allerton Conference on, Allerton, 2012.

[35] A. Sayed, S.-Y. Tu, J. Chen, X. Zhao and Z. Towfic, "Diffusion strategies for adaptation and learning over networks," Signal Processing Magazine, IEEE, vol. 30, no. 3, pp. 155-171, 2013. 
[36] Z. Towfic and A. Sayed, "Adaptive Penalty-Based Distributed Stochastic Convex Optimization," Signal Processing, IEEE Transactions on, vol. 62, no. 15, pp. 3924-3938, 2014.

[37] G. C. D. G. Fabio Bellifemine, Developing Multi-Agent Systems with JADE, West Sussex, England: John Wiley \& Sons Ltd, 2007.

[38] International, Digi, "XBee-PRO 900HP - Digi International," [Online]. Available: http://www.digi.com/products/xbee-rf-solutions/modules/xbee-pro900hp\#specifications.

[39] NREL, "Solar Prospector | maps.nrel.gov," [Online]. Available: http://maps.nrel.gov/prospector.

[40] Inerjy, "Inerjy Ecovert," [Online]. Available: http://www.inerjy.com/ecovert/.

[41] IEEE Std 1547.4-2011, IEEE Guide for Design, Operation, and Integration of Distributed Resource Island Systems with Electric Power Systems.

[42] OPC Foundation, "OPC Foundation," [Online]. Available: https://opcfoundation.org.

[43] M. Cintuglu, R. de Azevedo, T. M. and O. Mohammed, "Real-Time Experimental Analysis for Protection and Control of Smart Substations," Innovative Smart Grid Technologies Latin America (ISGT LA), 2015 IEEE PES Conference On, 2015.

[44] R. De Azevedo and O. Mohammed, "Profit-maximizing utility-scale hybrid wind-PV farm modeling and optimization," IEEE SoutheastCon, pp. 1-8, 2015.

[45] R. De Azevedo, D. Allen, Y. Perpuly and A. Sarwat, "PMU placement considering data uncertainty and redundancy," IEEE SoutheastCon, pp. 1-6, 2015.

[46] R. De Azevedo, M. Cintuglu, T. M. and O. Mohammed, "Multi-Agent Based Optimal Microgrid Control Using Fully Distributed Diffusion Strategy," Smart Grid, IEEE Transactions On (Submitted), 2016. 
VITA

RICARDO DE AZEVEDO

Born, Caracas, Venezuela

2004-2008

B.S., Computer and Electrical Engineering, Rice University, Houston, TX

2008

Lehman Brothers, New York, NY

2008-2010

Barclays Capital, New York, NY

2010-2011

Goldman Sachs, New York, NY

2011-2016

Lake Park Group, Orlando, FL

2014-2016

M.S., Electrical Engineering, Florida

International University, Miami, FL

\section{SELECTED PUBLICATIONS AND PRESENTATIONS}

1. De Azevedo, R., Cintuglu, M., M., T., \& Mohammed, O. (2016). Multi-Agent Based Optimal Microgrid Control Using Fully Distributed Diffusion Strategy. Smart Grid, IEEE Transactions On (Submitted).

2. Cintuglu, M., De Azevedo, R., M., T., \& Mohammed, O. (2015). Real-Time Experimental Analysis for Protection and Control of Smart Substations. Innovative Smart Grid Technologies Latin America (ISGT LA), 2015 IEEE PES Conference On.

3. De Azevedo, R., \& Mohammed, O. (2015). Profit-maximizing utility-scale hybrid wind-PV farm modeling and optimization. IEEE SoutheastCon, 1-8.

4. De Azevedo, R., Allen, D., Perpuly, Y., \& Sarwat, A. (2015). PMU placement considering data uncertainty and redundancy. IEEE SoutheastCon, 1-6. 